\title{
WestVirginiaUniversity
}

THE RESEARCH REPOSITORY @ WVU

Graduate Theses, Dissertations, and Problem Reports

2010

\section{Regulation of anoikis by ankyrin complexes}

\author{
Sanjeev Kumar \\ West Virginia University
}

Follow this and additional works at: https://researchrepository.wvu.edu/etd

\section{Recommended Citation}

Kumar, Sanjeev, "Regulation of anoikis by ankyrin complexes" (2010). Graduate Theses, Dissertations, and Problem Reports. 4620.

https://researchrepository.wvu.edu/etd/4620

This Dissertation is protected by copyright and/or related rights. It has been brought to you by the The Research Repository @ WVU with permission from the rights-holder(s). You are free to use this Dissertation in any way that is permitted by the copyright and related rights legislation that applies to your use. For other uses you must obtain permission from the rights-holder(s) directly, unless additional rights are indicated by a Creative Commons license in the record and/ or on the work itself. This Dissertation has been accepted for inclusion in WVU Graduate Theses, Dissertations, and Problem Reports collection by an authorized administrator of The Research Repository @ WVU.

For more information, please contact researchrepository@mail.wvu.edu. 
REGULATION OF ANOIKIS BY ANKYRIN COMPLEXES

Sanjeev Kumar

Dissertation submitted to the

School of Medicine at West Virginia University in partial fulfillment of the requirements for the degree of

Doctor of Philosophy

in

Biochemistry and Molecular Biology

Steven Frisch, Ph.D., Chair

Brad Hillgartner, Ph.D.

Fred Minnear, Ph.D.

Lisa Salati, Ph.D.

Michael Schaller, Ph.D.

Department of Biochemistry

Morgantown, West Virginia

2010

Keywords: Ankyrin, Anoikis, EMT, NRAGE, TBX2, E-cadherin, RIP1 Copyright 2010 Sanjeev Kumar 


\section{Abstract \\ Regulation of Anoikis by ankyrin complexes}

\section{Sanjeev Kumar}

Anoikis is a subset of apoptosis, suppressed by cell-extracellular matrix association. It is a safeguard mechanism against tumor metastasis. The present study was designed to understand the role of cortical cytoskeletal protein ankyrin- $G$ and its interacting proteins in anoikis. E-cadherin and ankyrin-G are function partners and during epithelial-mesenchymal-transition (EMT), both gets downregulated. During cellextracellular matrix dissociation, ankyrin-G shifts to cytoplasm from cytoskeleton and loses spectrin interaction. Overexpression of ankyrin-G induced apoptosis in death domain dependent manner, promoted by the presence of ankyrin-G death domain interacting partner, RIP1 (Receptor Interacting Protein with death domain). Ankyrin-G also interacted with a MAGE family protein NRAGE (Neurotrophin Receptor Interacting mAGE protein), which suppressed ankyrin-G induced apoptosis.

NRAGE suppressed anoikis and promoted anchorage independent growth. It was also upregulated in the cells which have undergone EMT as well as in various other tumor types. The oncogenic transcription repressor TBX2 (T-Box Protein) interacted with NRAGE and co-operated functionally in anoikis suppression and anchorage independent growth. Ankyrin-G sequestered NRAGE in cytoplasm and counteracted NRAGE mediated transcription activity. NRAGE also affected E-cadherin mediated adherens junction kinetics and activated pro-survival signaling in cells dissociated from extracellular matrix.

We report a novel pathway, controlled by E-cadherin and ankyrin-G, sequestering NRAGE in cytoplasm in epithelial cells. During EMT, loss of E-cadherin and ankyrin-G promotes NRAGE localization to nucleus. NRAGE co-operates with TBX2 in suppressing anoikis and promoting anchorage independent growth. 


\section{DEDICATION}

I would like to dedicate my dissertation to my mother and father (passed away on November 3 , 2006). I also dedicate this thesis to my brothers, sister and their families for providing unconditional support, encouragement throughout my life and also for taking care of parents when I could do nothing being so far away from home. 


\section{ACKNOWLEDGMENTS}

I would like to thank my mentor Dr. Steven Frisch for diligence guidance and encouragement. Thank you for training me to enhance my research abilities under your supervision and devoting your time and resources to my development. I would like to thank Dr. Lisa Salati, for all the help and suggestions provided before and after joining West Virginia University. I would also like to thank my committee members Dr. Brad Hillgartner, Dr. Fred Minnear, and Dr. Michael Schaller for valuable suggestions and comments.

I would like to thank Jane Schupp, who designed many constructs and cell lines for the present study, without which my thesis wouldn't have completed. I also thank my lab members Sun Park and Ben Cieply for all the co-operation and teamwork.

I would like to thank my friend Supriya, for being the best support system as long as we have known each other. I would also like to thank my friends Subha, Palani, Ratnesh, Saro and Yan Wang for all their help and support.

I would like to thank Department of Biochemistry and Cancer Center students, faculties and staffs for all the support. 


\section{TABLE OF CONTENTS}

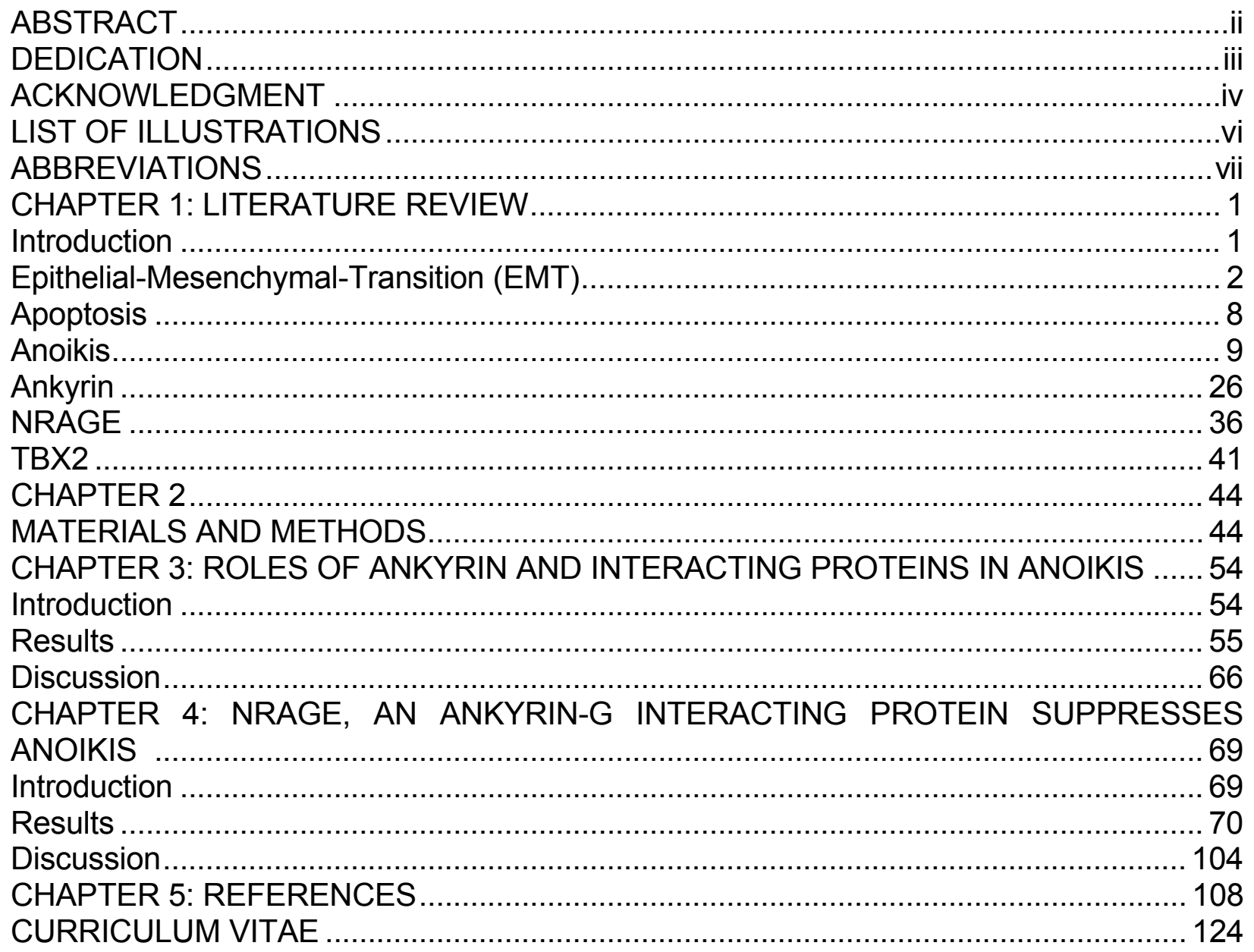




\section{LIST OF ILLUSTRATIONS}

\section{CHAPTER 1}

Figure1 Stages of cancer progression from normal epithelium to invasive carcinoma..... 2

Figure2 Epithelial-Mesenchymal-Transition ....................................................... 4

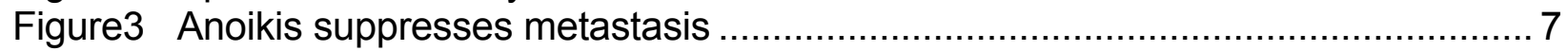

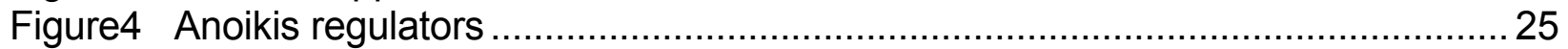

Figure5 Ankyrin links membrane proteins to actin cytoskeleton ................................ 30

Figure6 Ankyrin regulates various functions in epithelial cell ....................................... 36

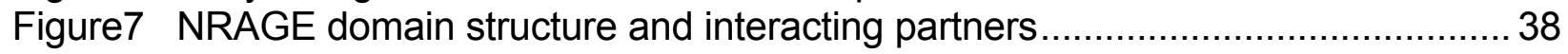

Figure8 NRAGE confers pro-apoptotic and pro-tumorigenic function............................. 40

\section{CHAPTER 3}

Figure1 Ankyrin-G is a functional E-cadherin partner ............................................ 56

Figure2 Ankyrin-G localization is affected by cell-matrix dissociation ..........................57

Figure3 Loss of cell-extracellular matrix interaction promotes dissociation of ankyrin-

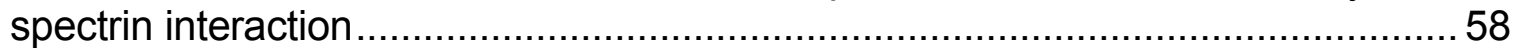

Figure4 The ZU5 and death domain of ankyrin-G are pro-apoptotic ..........................59

Figure5 Transient knockdown of ankyrin-G enhances anoikis..................................60

Figure6 The ankyrin-G lacking membrane binding domain suppresses anoikis..............61

Figure7 Ankyrin-G stabilizes EGFR in mammary epithelial cells .................................63

Figure8 Ankyrin-G interacts with death domain proteins RIP1 and TRADD ...................64

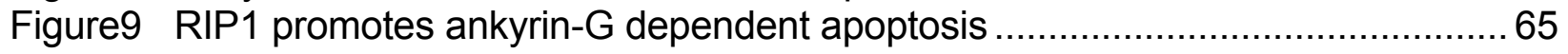

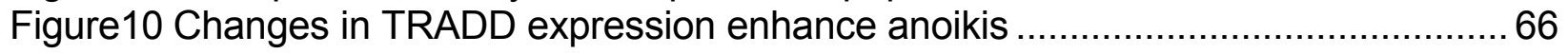

\section{CHAPTER 4}

Figure1 Ankyrin-G interacts with NRAGE in mammary epithelial cells .........................70

Figure2 Ankyrin-G and NRAGE interactions are specific........................................ 71

Figure3 NRAGE co-immunoprecipitate stably expressed exogenous ANK-105 in

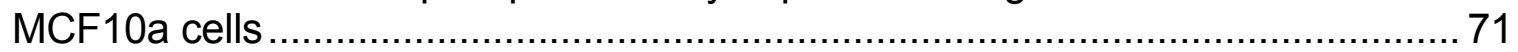

Figure4 The presence of C-insert interferes with ankyrin-G and NRAGE interaction..... 73

Figure5 Ankyrin-G requires MAGE and C-terminal domain for NRAGE interaction ........74

Figure6 ZU5 domains of ankyrin don't contribute to NRAGE interaction ........................75

Figure7 NRAGE interacts with UPA domain of ankyrin-G .................................... 76

Figure8 NRAGE affects cellular and biochemical characteristics of ankyrin-G ..............76

Figure9 NRAGE affects cell polarity by affecting adherens junction kinetics..................78

Figure10 NRAGE suppresses anoikis in epithelial and endothelial cells .........................79

Figure11 NRAGE level inversely correlates with anoikis sensitivity in mammary epithelial

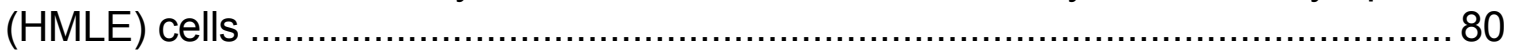

Figure12 NRAGE is overexpressed in certain tumor types...................................... 82

Figure13 Transient knockdown of NRAGE promotes anoikis in EMT cells ....................... 84

Figure14 NRAGE promotes anchorage independent growth in EMT/tumor cells ............. 86

Figure15 NRAGE interacts with TBX2 in MCF10a and HMLE cells ............................... 87

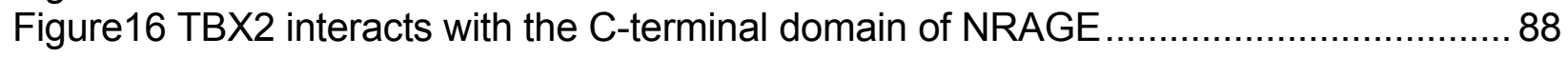

Figure17 TBX2 and NRAGE interactions are functionally significant.............................. 89

Figure18 NRAGE is required for TBX2-dependent anchorage-independent growth .........90 


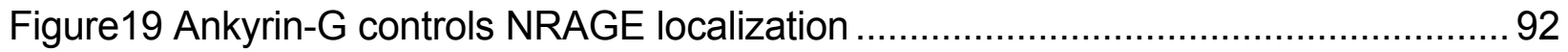

Figure20 Ankyrin-G affects the localization of exogenous NRAGE in MCF10a cells ....... 93

Figure21 EMT promotes nuclear accumulation of NRAGE....................................... 94

Figure22 The knockdown of ankyrin-G partially relocates exogenous NLS-NRAGE to

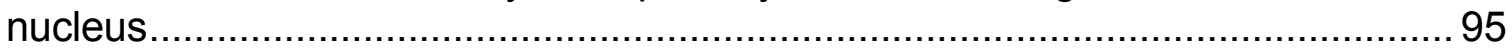

Figure23 NRAGE represses DIx-5 dependent transcription activity .............................96

Figure24 Ankyrin-G promotes DIx-5 mediated transcription activity .............................97

Figure25 The tumor suppressor p19-ARF promotes anoikis ....................................... 98

Figure26 NRAGE effect on anoikis requires ARF .................................................. 99

Figure27 Expression of nuclear NRAGE suppresses anoikis ................................... 100

Figure28 Cytoplasmic NRAGE suppresses anoikis in HMLE and MCF10a cells ........... 101

Figure29 NRAGE promotes survival through activated AKT ...................................... 102

Figure30 NRAGE overexpression activate signaling events during cell-extracellular matrix dissociation .................................................................................................. 103

Figure31 NRAGE suppresses anoikis and anchorage independent growth by regulating various cellular processes ....................................................................... 105 


\section{ABBREVIATIONS}

ankDD Death domain of ankyrin

AR Ankyrin repeats

ATP adenosine triphosphate

C

Celcius

DD

Death Domain

DMEM

Dulbecco's modified Eagle's medium

DMSO

Dimethyl Sulfoxide

EGF

Epidermal growth factor

EGFR

Epidermal growth factor receptor

EMT

Epithelial-Mesenchymal-Transition

Erk

Extracellular signal-regulated kinase

FADD Fas-associated death domain

FAK

Focal adhesion kinase

FBS

Fetal bovine serum

GFP Green fluorescent protein

HBSS Hank's balanced salt solution

HGF Hepatocyte growth factor

kDa KiloDalton

MAGE Melanoma Antigen

MEF Mouse embryo fibroblast

MET Mesenchymal-Epithelial-Transition

MMP Matrix metalloproteinase 


$\begin{array}{ll}\text { NF-kB } & \text { Nuclear factor-kappa B } \\ \text { PBS } & \text { Phosphate buffered saline } \\ \text { PDGF } & \text { Platelet Derived Growth Factor } \\ \text { PIDD } & \text { p53-induced protein with death domain } \\ \text { pMIG } & \text { MSCV-IRES-GFP retrovial construct } \\ \text { p85 } & \text { 85kDa subunit of pl3K } \\ \text { PKC } & \text { Protein kinase C } \\ \text { RIP1 } & \text { Receptor interacting protein with death domain } \\ \text { TGF beta } & \text { Transformation growth factor-beta } \\ \text { TNF } & \text { Tumor Necrosis Factor } \\ \text { TRADD } & \text { TNF Receptor interacting adaptor protein containg death domain } \\ \text { UPA } & \text { Domain conserved in Unc5, PIDD and ankyrin } \\ \text { WT } & \text { Wild type } \\ \text { ZU5 } & \text { Domain conserved in Unc5 and ZO1 proteins }\end{array}$




\section{CHAPTER 1}

\section{LITERATURE REVIEW}

Introduction

An epithelial cell is characterized by its apical-basolateral polarity and the basic framework is supported by basement membrane or extracellular matrix (ECM) and cellcell junctions. The cell-cell and cell-membrane interactions involve various junction proteins which are known to control their assembly into tissues and organs through a process called cell adhesion. The adhesion properties of cells are supported and regulated by the cytoskeleton which links the cytoplasm to specialized junctions in plasma membrane. The epithelial cell homeostasis is tightly regulated by features such as cellcell contact inhibition and requirement for growth signal. The epithelial cells such as in the gastro-intestinal tract, mammary and prostate glands have very high turnover rate and any defects either constitutively or during tissue remodeling affecting cell homeostasis, results into uncontrolled cell proliferation and development into cell carcinoma. Cell carcinomas (cancer arising from epithelial cells) account for more than $90 \%$ of human cancer (Hanahan and Weinberg, 2000).

Cancer cells are defined by traits such as loss of contact inhibition and self sufficiency in growth. There are varieties of defects in individual cells caused either by genetic alterations or misregulation of signaling pathways. The origin of cancer cells can be traced to a single primary tumor which has gone through heritable changes enables them to outgrow their neighbors. The primary tumor is called benign as they can still be cured by removing the mass surgically whereas aggressive and invasive form known as malignant tumor have acquired the ability to form secondary tumors at distant micro- 
environment. The malignant tumors can invade surrounding tissues, enter the bloodstream or lymphatic vessels and result into cell metastasis. The progression of primary tumor to metastatic tumor requires multiple steps through a process known as epithelial-mesenchymal-transition (EMT) (Fig.1) (Kalluri and Weinberg, 2009).

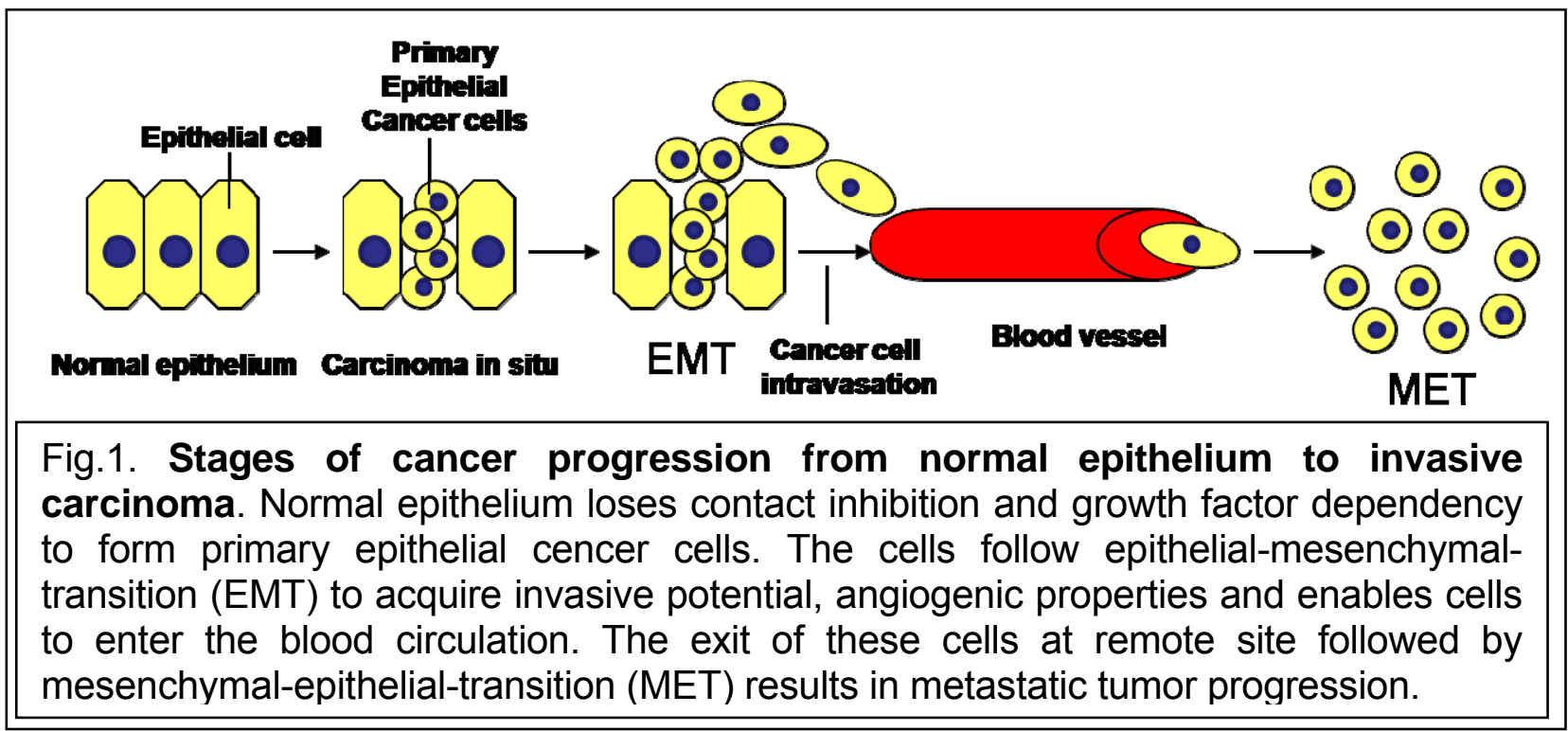

Epithelial-Mesenchymal-Transition (EMT)

EMT is a process during which an epithelial cell (polarized cell with cell-cell and cell-extracellular matrix interactions) undergoes multiple biochemical changes to acquire mesenchymal phenotype. During this process, cells have acquired characteristics like cellular migration and cell invasion and the cells become resistant to apoptosis which allow them to survive in metastatic secondary attachment site. EMT is a natural phenomenon in certain cases such as implantation, embryo formation and organ development as well as in wound healing, tissue regeneration, and organ fibrosis. During cancer progression, EMT brings out the life-threatening changes resulting into secondary carcinoma from the primary malignant cancer cells. Based on the type of changes and 
tissues involved, three types of EMT have been characterized (Kalluri and Weinberg, 2009).

Type 1 EMT: This type of EMT has been described during implantation, embryo formation, and organ development. During this process, diverse cell types are generated which shares common mesenchymal features. The process begins with gastrulation where fertilized eggs develop into three germ layers. The epiblast layer exhibits apicalbasal polarity as a result of E-cadherin expression. The mesenchymal transition activates wnt signaling pathways, which in turn upregulates EMT genes such as snail, well characterized for its role in E-cadherin repression. The EMT is completed by a process called MET (mesenchymal-epithelial-transition) which is involved in generating secondary epithelia (Vicovac and Aplin, 1996).

Type 2 EMT: This type of EMT process has been described during wound healing, tissue regeneration, and organ fibrosis. The repair-associated events generate fibroblast and other related cells and the tissue is reconstructed following trauma and inflammatory injury. This event has also been described during kidney, liver, lung, and intestine regeneration. The extra-cellular matrix proteins such as collagen, laminins and vimentin contribute to this process efficiently (Rastaldi et al., 2002).

Type 3 EMT: The type 3 EMT describes the event during tumor progression and metastasis. The genetic/epigenetic changes affect oncogene and tumor suppressors that result in neoplastic cell growth and favor clonal outgrowth for the development of localized tumors. These localized tumors are characterized by increased proliferation and angiogenesis (formation of networks of blood vessels). During EMT, a multi-step invasiveness occurs through basement membrane which results in metastatic 
dissemination. The EMT cells start expressing mesenchymal proteins such as vimentin and desmin and the process includes intravasation, transport through circulation, extravasation, formation of micro-metastases and colonization. It is also believed that the genetic/epigenetic changes are more responsive to EMT-inducing heterotypic signal originated in the tumor mediated stroma. EMT also inhibits oncogene-mediated senescence to cause aggressive dissemination. The EMT signals originated from stroma include growth factors such as HGF, EGF, PDGF, and TGF-beta etc. These signals activate EMT genes such as snail, slug, ZEB1, and Twist etc which in turn promote complex EMT reprogramming. The reprogramming activates signaling pathways including ERK, MAPK, PI3K as well as receptor proteins promoting adhesion to extracellular proteins (integrins). The reprogramming of these cells results in disruption of adherens junctions and promotes cell-ECM adhesions by integrins (Thiery, 2002). For the purpose of the present review, cancer relevant EMT will be referred to as general EMT.

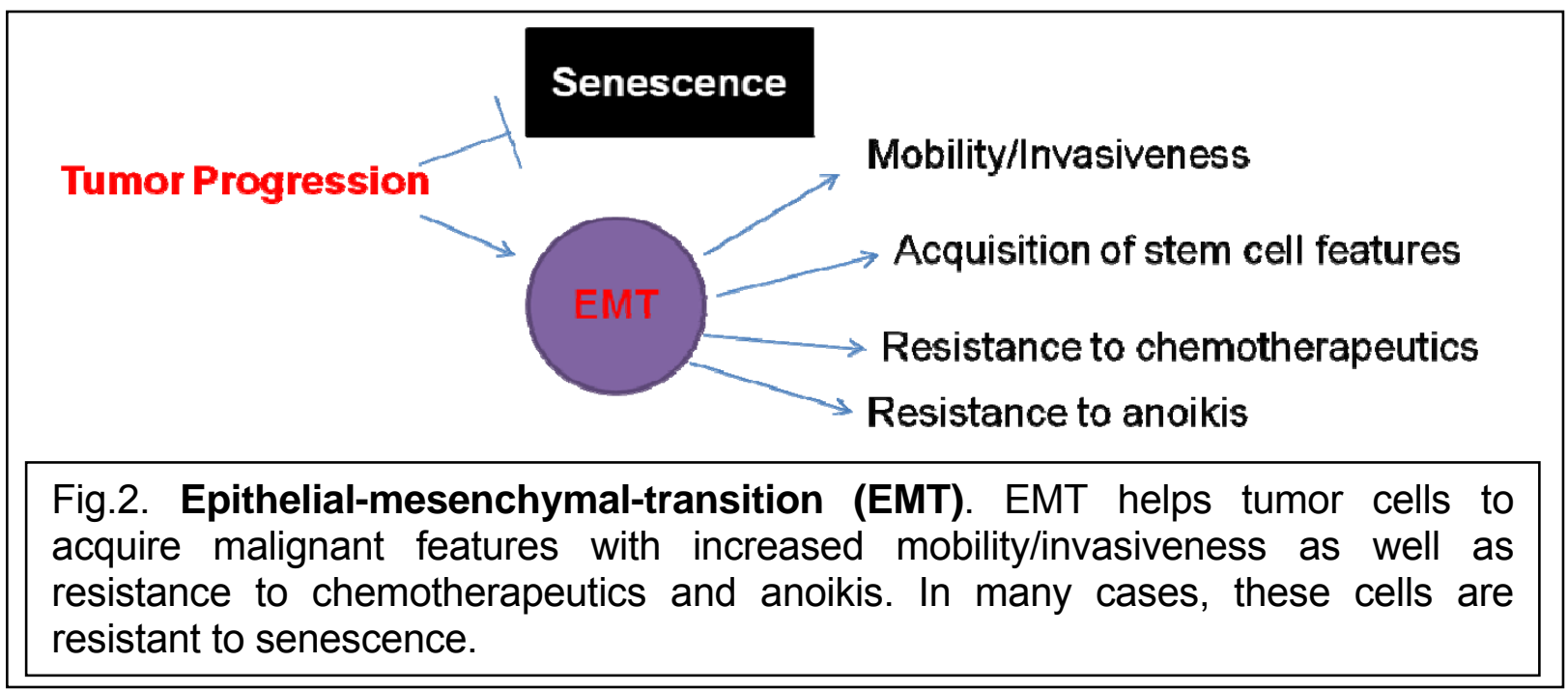

The tumors can originate from various heritable cellular defects. The affected cells can be reprogrammed for epithelial-mesenchymal-transition (EMT) by various factors. 
The cell characteristics during EMT show common features i.e. they lose epithelial nature (loss of E-cadherin) and start behaving like mesenchymal cells (expression of $\mathrm{N}$-cadherin, fibronectin, and vimentin etc).

The common features for EMT cells include (i) increased invasiveness/motility, (ii) acquisition of stem-cell features, (iii) resistance to chemotherapeutics, and (iv) resistance to anoikis (Fig. 2) (Weinberg, 2008).

Increased invasiveness/motility: Cell motility/invasiveness is a critical component of EMT. The mesenchymal cells have increased motility/invasiveness properties, as a result of integrin-mediated responses to the extracellular matrix (Keely et al., 1997). This event correlates with actin cytoskeleton reorganization mediated by small GTPases such as Cdc42 and Rac. The migration occurs through a well identified processes including (a) development of polarized cell projections, (b) focal adhesions mediated projection stabilization (c) cell contraction, and (d) forward cell migration as a result of rear end detachment. Various growth factors (EGF), cytoskeletal (spectrin), integrins (integrin and FAK), proteases (MMPs, calpains etc.), and signaling (ERK, MEK etc.) components collaborate for this process.

Acquisition of stem-cell features: During EMT, various pleiotropically acting transcription factors overexpress, which are otherwise known to play critical roles in embryogenesis, develop malignant traits. These transcription factors (Twist, Snail, Slug, goosecoid, FoxC2, ZEB1, and ZEB2/Sip1 etc) can reprogram to a less differentiated cell with self-renewal properties. In experimental set up, these EMT-transcription factors are capable of inducing expression of other members of this group (Mani et al., 2008). 
Resistance to chemotherapeutics: There is increasing evidence that the cancer cells which have undergone EMT, show increased resistance to apoptosis and chemotherapeutics drugs. Snail overexpression confers resistant to TNF-alpha induced apoptosis whereas slug null mutant mice in contrast show an increased sensitivity to the death induced by gamma-irradiation (Vega et al., 2004), (Inoue et al., 2002) and (PerezLosada et al., 2003). TGF-beta and other family members promote EMT by inducing snail/slug expression in hepatocytes, epithelial and mesothelial cells. Various tumor types have been reported to acquire Paclitaxel (a taxol group of drug, known to target microtubules and can arrest cells in mitosis) resistance as a result of Twist1 gene amplification (Wang et al., 2004). The breast cancer cells systematically selected for highly invasive phenotype promote Paclitaxel resistance by the virtue of Twist1 mediated transcriptional reprogramming (Cheng G.Z. et al., 2007). The DNA damaging drug Adriamycin, frequently used in cancer therapeutics, is known to function through p53 mediated pathway (Saito et al., 2003). Adriamycin treatment resistant breast cancer and ovarian cancer cells displayed EMT features and upregulated Twist1, correlates with multidrug resistance and increased invasive potential (Li et al., 2009). A panel of pancreatic cancer cells such as AsPC-1, PANC-1 etc. showed resistance to various chemotherapeutic drugs such as Genicitabine, Cisplatin etc. through induction of ZEB1 dependent EMT reprogramming (Arumugam et al., 2009). The resistance to apoptotic and chemotherapeutic drugs result in therapy failure and reduced survival for cancer patients. For the better therapeutic approach, it is essential to understand the molecular mechanisms underlying drug resistance. The drugs reversing EMT used in combination with existing therapeutic agents could be better therapeutic interventions. 
Resistance to anoikis: Anoikis (or homelessness in Greek) is a subtype of apoptosis which is inhibited by cell-extracellular matrix interaction (Frisch and Francis, 1994). This safeguard mechanism is the basic feature of normal epithelial cells, which in turn suppresses tumorigenic/metastatic behavior (Fig.3). Anoikis helps maintain the homeostasis in tissues with high turnover rate such as the gastrointestinal tract, mammary and prostate glands. Any misregulation during the anoikis process could increase colonizing property and formation of secondary metastatic tumors. The EMT cells acquire the characteristics of mesenchymal cells and they frequently lose epithelial markers. Mesenchymal cells by nature are resistant to anoikis as they do not need extracellular matrix for survival. Anoikis is strictly a sub-set of apoptosis. To understand the process of anoikis better, we should review apoptosis and its contributors which are also critical for anoikis.

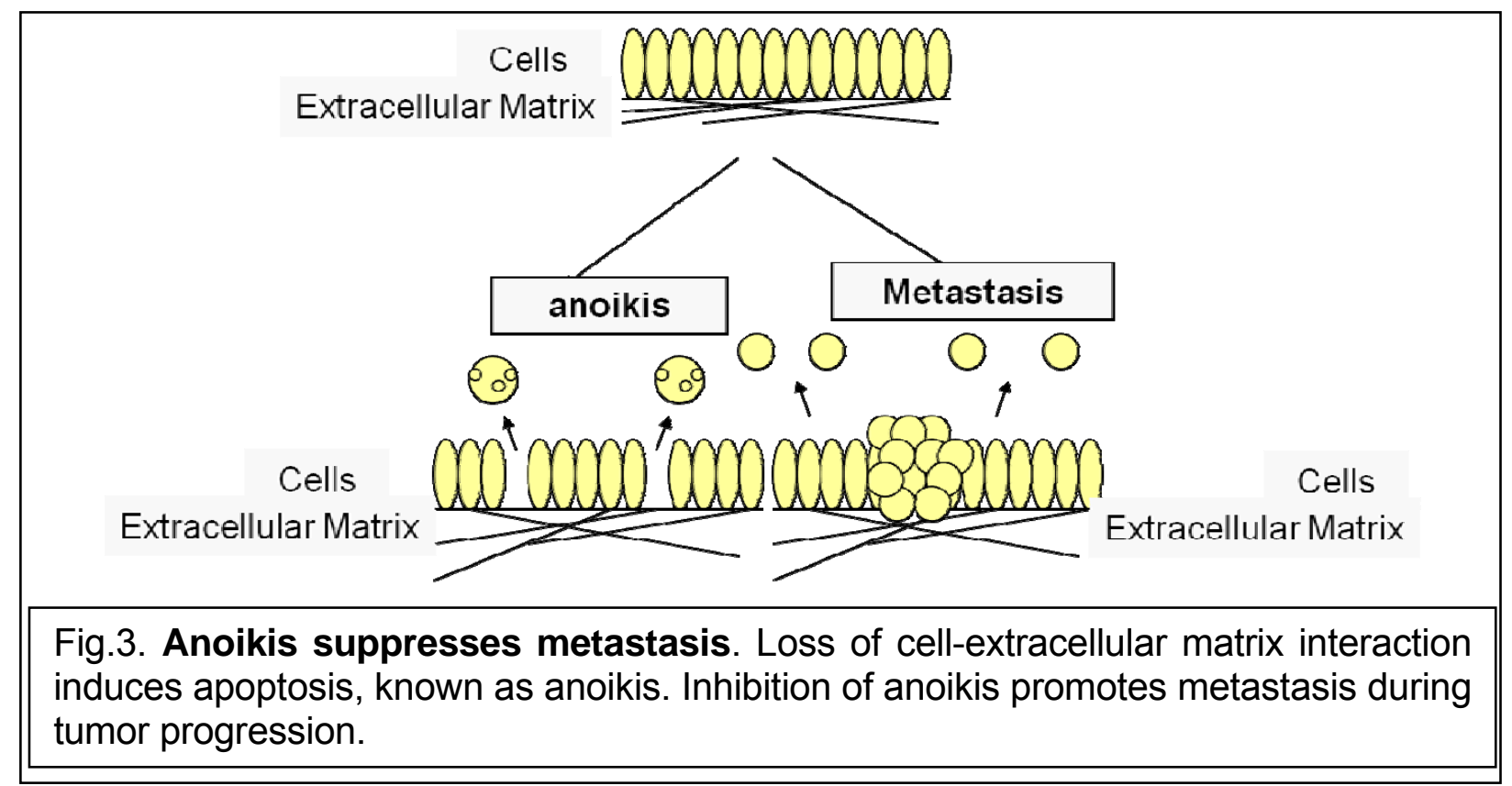


Apoptosis

Apoptosis is a descriptive name given to the process of programmed cell death in eukaryotes. Apoptotic cell suicide is a fundamentally important biological process that is required to maintain the integrity and homeostasis of multi-cellular organisms. The apoptotic response in cells can be activated by several physiological conditions such as DNA damage induced by cytotoxic stress, lack of growth factor receptor (GFR) signaling, binding of death ligands such as FasL and TNF to the respective death receptors (DR) and loss of cellular contact with extra-cellular matrix (anoikis). All the different pathways share many common components. During apoptosis, the cell activates an intrinsic suicide mechanisms which systematically traces the cell, surface membrane begins to bleb, cell expresses prophagocytic signals, cells start to shrink, and severs contact with its neighbors. The nucleus shows condensed and cleaved chromatin (ensuring genetic death of the affected cell) and eventually the whole cell fragments are engulfed into the membrane bound vesicles that are rapidly ingested by neighboring cells. The apoptotic process is extremely rapid (typically a few minutes to few hours), and the apoptotic debris is cleared away with similar rapidity. Programmed cell death occurs in most animal tissues to eliminate harmful cells or those produced in excess (Fraser and Evan, 1996) (Enari et al., 1995). Apoptotic signals are generally characterized by two major pathways: Extrinsic or Intrinsic. The extrinsic pathways include activation of death receptors such as TNF receptor or FAS by their ligands such as TNF or FasL. When three death ligands bind to their respective homotrimeric receptors, the receptor death domains cluster to recruit adaptor proteins such as FADD (FAS-associated Death Domain protein) and TRADD (TNFR1-associated Death Domain protein). These adaptor proteins have death 
domains and in turn recruit the initiator caspases such as caspase- 8 through death effector domain (DED). The initiator caspases in turn activates the effector caspases such as caspase-3/-7 (Ashkenazi and Dixit, 1998). The cellular stress induced intrinsic pathway is mediated by Bcl-2 (CED-9 homolog) family members. The interplay of Bcl-2 family member proteins regulates the mitochondrial integrity and the apoptosis process promotes release of mitochondrial components required for activating terminal caspases. Bcl-2 family members can either be pro-apoptotic or anti-apoptotic. They possess either one of the four conserved motifs known as the $\mathrm{Bcl}-2$ homology $(\mathrm{BH})$ domains, $\mathrm{BH} 1, \mathrm{BH} 2$, $\mathrm{BH} 3$ and $\mathrm{BH} 4$. The distinguishing feature of this family is the ability to form homodimers and heterodimers. In normal physiological condition, the anti-apoptotic Bcl-2 family proteins (e.g. Bcl-2 and $\mathrm{Bcl}-\mathrm{xl}$ etc.) remain localized to the mitochondria to keep the mitochondria intact. Under cytotoxic stress, the change in physiological status is sensed by pro-apoptotic Bcl-2 family proteins (Bax, Bak, and Bok etc.), which in turn titrates out the anti-apoptotic Bcl-2 family member proteins. The activated pro-apoptotic $\mathrm{Bcl}-2$ family proteins localize to the mitochondrial membrane, leading to mitochondrial permeabilization that results in generation of reactive oxygen species, loss of ATP production, release of cytochrome $\mathrm{c}$ and the apoptosis inducing factor (AIF). One of the released proteins, cytochrome $\mathrm{c}$ forms a complex with Apaf-1 (CED-4 homolog), which in turn activates the initiator caspase, caspase-9 (Adams and Cory, 1998). Caspase-2 has also been shown to be required for the pre-mitochondrial permeabilization (Lassus et al., 2002). Once the initiator caspases are activated, they process and activate the downstream caspases such as caspase- $3,-6$ and -7 . The activation of terminal caspases acts as point of no return for the ongoing apoptotic event. Extrinsic pathway also activates 
mitochondrial permeabilization through activation of caspase-8, which results in cleavage of Bid into tBid, in turn stimulates the release of cytochrome $\mathrm{c}$ from the mitochondria and amplification of caspase cascade (Boatright and Salvesen, 2003). In anoikis, extrinsic and intrinsic pathway members also contribute by a process initiated by integrins and other adhesion molecules, cytoskeletal re-organization with various signaling and transcriptional reprogramming.

Anoikis

As mentioned previously, anoikis is induced by cell-extracellular matrix dissociation or engagement of inappropriate matrix. By definition, anoikis is the result of interplay between cell-matrix interactions and is therefore, regulated by core apoptotic molecules, cell-matrix and cell-cell adhesion molecules (such as integrins, non-integrins and cadherins) as well as various receptor kinases, Rho GTPases and nuclear factors.

Bcl-2 family members: The interplay between pro-apoptotic and anti-apoptotic Bcl2 family proteins regulate the intrinsic pathway of apoptosis through the control of mitochondrial integrity. The preliminary characterization of anoikis indicated that the MDCK, HaCat and E1A reverse transformed $\mathrm{HT} 1080$ cells overexpressing Bcl-2 resisted anoikis (Frisch and Francis, 1994). Other Bcl-2 family members such as Bmf (Bcl-2 modifying factor), Bim, Bad and Mcl-1 have also been implicated in the regulation of anoikis response. The proapoptotic $\mathrm{BH} 3$-only protein $\mathrm{Bmf}$, interacts with dynein light chain-2, a component of myosin $\mathrm{V}$ complex, and remain sequestered until anoikis induction or treatment of cytochalasin D treatment allows Bmf to translocate and titrate out Bcl-2 (Puthalakath et al., 2001). In 3-D morphogenesis assay, Bmf is reported to be required for lumen formation (a process similar to anoikis) (Schmelzle et al., 2007). 
Similarly, the pro-apoptotic $\mathrm{BH} 3-$ only protein Bim interacts with cytoplasmic dynein light chain (DLC1), a component of microtubule associated dynein motor complex and remain sequestered until the apoptotic stimuli (Puthalakath et al., 2001). The co-ordinated regulations of Bim by integrins and EGFR have been implicated in suppressing anoikis. Integrins and EGFR activate ERK/MAPK pathway, leading to Bim phosphorylation, and its degradation. Bim expression is found to be induced in cells detached from extracellular matrix (Biswas and Greene, 2002; Ley et al., 2004; Reginato et al., 2003). Interestingly, the pro-anoikis signaling cascade involving JNKs have been reported to phosphorylate Bim and Bmf, affecting their associations with dynein or myosin $\mathrm{V}$ respectively, and resulting in cytochrome $\mathrm{c}$ release. This suggests a mechanistic connection between JNKs and downstream events that lead to anoikis (Lei and Davis, 2003). Another anti-apoptotic Bcl-2 family member, Mcl-1 is shown to overexpress in melanomas have been shown to overexpress in melanomas. The overexpression of Mcl1 is the result of mutated B-RAF (50-70\% melanomas) and activated MEK/ERK pathway. The transient knockdown of Mcl-1 (using RNA interference) renders mutant B-RAF melanoma cells sensitive to anoikis. This observation suggests that the $\mathrm{BH} 3$ mimetic compounds disrupting fibronectin-integrin signaling could be used for therapeutic approaches (Boisvert-Adamo et al., 2009).

Death Receptor Signaling Pathway: Death Receptor or extrinsic pathway is critical for apoptosis. At present, there is very limited evidence suggesting the involvement of this pathway in anoikis. The expression of dominant negative form of FADD suppressed anoikis by blocking caspase-8 recruitment at the DISC (death-inducing signaling complex) (Frisch, 1999; Rytomaa et al., 1999). Although, these data support the 
involvement of extrinsic pathway, the direct involvement of death receptor is yet to be demonstrated. The extrinsic pathway involvement didn't require external ligand activation and soluble extracellular domains of Fas, TNF-alpha receptor and death receptor 5 (DR5), which would act as dominant negative form for receptor activation, failed to inhibit anoikis (Rytomaa et al., 1999). The induction of anoikis was suppressed by expression of Bcl-2, suggesting that the caspase-8 activation was result of an activated intrinsic apoptotic pathway. During 3-D morphogenesis assay, the tumor necrosis factor-related apoptosis-inducing ligand (TRAIL) was found to be up-regulated, but dominant negative forms of FADD or TRAIL didn't rescue the luminal clearing during the early stages of acini clearing (Mills et al., 2004). In contrary, anoikis induced in endothelial cells resulted in overexpression of Fas and Fas-L and massive downregulation of Fas pathway inhibitor, FLICE inhibitory protein (FLIP) indicates the involvement of extrinsic pathway (Aoudjit and Vuori, 2001). At present, it is believed that death receptor pathway could be the result of positive feed-back mechanism, secondary to mitochondrial permeabilization, which in part is supported by the $\mathrm{Bcl}-2$ and $\mathrm{Bcl}-\mathrm{xL}$ mediated inhibition of caspase- 8 activation (Grossmann, 2002).

Integrins dependent survival pathways: Integrins are the cell receptors known to link cells to basement membrane through interaction with extracellular matrix proteins such as fibronectin, collagen and laminin. Integrins function as the integrators of the extracellular matrix and cytoskeleton. Integrins are a large family of cell surface receptors expressed from sponges to mammals. They are combination of two subunits, alpha and beta. There are 8 known beta subunits which can assort with 18 different alpha subunits to form 24 distinct integrins. All integrins have their own binding specificity and signaling 
properties. Most of the integrins recognize several extracellular matrix proteins with variable specificity. They are generally characterized on the basis of evolutionary relationships, binding specificities and restricted expression. Upon binding to extracellular matrix, integrins become clustered at cell membrane and associate with the cytoskeleton and signaling complexes that promote the actin (or cytokeratin) assembly. The integrins attachment to extracellular matrix provides the survival signal to the cell which in turn activates the signaling pathway for cell proliferation and differentiation (Frisch and Ruoslahti, 1997). Integrin-extracellular signaling has been shown to play key roles in development, immune responses, leukocyte traffic, hemostasis, and cancer. They are central to many genetic and auto-immune diseases. By definition, anoikis is a form of apoptosis induced by loss of cell-extracellular matrix interaction or cell interaction with inappropriate matrix. Anoikis can be mimicked intracellularly by cell adhesion signaling pathway interference such as overexpression of unliganded, dominant negative form of integrins, perturbation of actin cytoskeleton and interfering integrin-extracellular interaction by RGD (arginine-glycine-aspartate motif of extracellular matrix proteins, required for integrins binding) peptide. The integrins and their interacting partners, involved in cell signaling, cytoskeleton organization and cell migration have been demonstrated for their role in anoikis regulation. The ligands require specific integrins for survival signaling as surfaces coated with fibronectin and anti-beta2 integrin compared to polylysine protected endothelial cells (Meredith et al., 1993). Similarly, mammary epithelial cells are also protected by cell basement membrane compared to collagen and fibronectin matrices which failed to provide the protection (Boudreau et al., 1995). Other reports also suggested that the failure of integrin mediated protection was a result of 
inappropriate signaling rather than ligand attachment to the integrins (Howlett et al., 1995). The integrin molecules differ in their ability to suppress anoikis in a single cell type and alternatively, tumor cells express aberrant integrin types (Juliano and Varner, 1993). The integrin-mediated survival is the result of activation of signaling pathways which otherwise can be aberrant in tumor cells.

The integrin-extracellular matrix association results in clustering and recruitment of various adaptor proteins which in turn connect with the cytoskeleton, cytoplasmic kinases and transmembrane growth factor receptors. The cytoskeletal proteins talin and paxillin bind to cytoplasmic tail of integrin beta-subunits, resulting in recruitment and autoactivation of focal adhesion kinase (FAK) by phosphorylation at $\mathrm{Tyr}^{397}$. This phosphorylation creates a binding site for src homology domain 2 (SH2) of Src or Fyn. The Src kinases in turn phosphorylate and activate various focal adhesion components such as cytoskeletal protein paxillin, tensin and the docking protein p130CAS, which recruits other adaptor proteins Crk and Nck. FAK is overexpressed in many tumor types (breast, colon, lung and squamous cell carcinomas). Another kinase from focal adhesion complex, ILK (integrin linked kinase) has also been reported for its role in integrin signaling. The role of ILK was attributed to its hyperactivity in cells with aberrantly activated phosphatidyl inositol (PI)-3-kinase or dysfunction of the tumor suppressor PTEN, correlated with ILK activation by phospholipids (Wu et al., 2001). ILK expression also increased in prostatic adenocarcinoma in stage dependent manner (Graff et al., 2001). ILK dependent Akt/PKB activation may be a major mechanism of anoikis suppression. ILK activity increases upon cell adhesion to matrix and decreases upon cellextracellular matrix dissociation (Attwell et al., 2000). Recently, ILK has also been 
demonstrated for preventing anoikis in human mesenchymal stem cells (Benoit et al., 2007).

Integrin signaling can activate FAK and suppress anoikis (Frisch et al., 1996). The activated form of FAK protects cells from anoikis through activation of extracellular signal regulated protein kinases (ERKs) pathway by activating ras as well as promoting enzymatic activity of phosphatidyl-inositol-3'-phosphate kinase (PI3K) by p85 interaction. The ERK pathway in turn inactivates pro-apoptotic Bcl-2 family protein BAD (phosphorylation by ERK target kinase, Rsk), caspase 9 and transcription factors which induce c-fos and cyclin D1 expression (Abbi and Guan, 2002).

FAK is also shown to suppress anoikis by interaction with cytoskeletal protein paxillin and death domain containing kinase receptor interacting protein (RIP1). Paxillin binding to FAK activates the pro-survival mitogen activated protein kinase (MAPK) signaling pathway. A focal adhesion and adheren junction protein vinculin interacts with the leucine-rich repeat region (LD motifs) of paxillin and interfere with the FAK interaction. Vinculin-deficient cells are resistant to anoikis and this defect can be restored by reexpression of the paxillin interacting carboxy-terminal tail of vinculin (Subauste et al., 2004). The vinculin-mediated interference of FAK-paxillin interaction negatively regulates ERK activation and restores caspase 9 activity, suggesting a novel mechanism for the regulation of FAK-mediated cell survival by a cytoskeletal component. Vinculin is downregulated in highly metastatic tumor cells and its re-expression has previously been shown to suppress cancer cell motility and tumor metastasis (Rodriguez Fernandez et al., 1993). The inhibition of FAK phosphorylation (by kinase inhibitor staurosporine) promoted its degradation and RIP1-dependent apoptosis (Kurenova et al., 2004). RIP1, interacting 
partner for death domain proteins FADD and TRADD, is a well characterized protein for its role in death receptor signaling pathway by either activating NF-kB survival pathway or caspase 8 mediated pro-apoptotic pathway (Lin et al., 1999). The dominant negative form (carboxy-terminal domain) of FAK induces apoptosis which is attenuated by loss of RIP1. This study also demonstrated that RIP1-null mouse embryo fibroblast or the transient knockdown of RIP1 resulted in anoikis resistance (Kurenova et al., 2004). In another study, loss of cell-extracellular matrix interaction or expression of dominant negative form of FAK resulted in mitochondrial localization of Bax which was counteracted by expression of PI3K or src overexpression (Gilmore et al., 2009). So, the adherent cells block apoptosis by impairing Bax mitochondrial localization through the combined action of FAK, $\mathrm{PI} 3 \mathrm{~K}$, and Src, which might otherwise be nonfunctional during anoikis (Playford et al., 2008).

The p130Cas family proteins, known to be phosphorylated by Src kinase and interact with the Crk family of adaptor proteins, also interact with FAK to regulate anoikis. The p130Cas and related protein, HEF1 (human enhancer of filamentation-1) interact with FAK PxxP domain. They get inactivated during the $G 2$ to $M$ transition, either via phosphorylation in case of $\mathrm{p} 130 \mathrm{Cas}$ or caspase-mediated cleavage in case of HEF1. The inactivation of these proteins might lead to loss of cell adhesion, required for the onset of mitosis. The p130Cas and HEF1 family proteins are also cleaved by caspases during death receptor-induced apoptosis. HEF1 overexpression or the caspase-cleaved form of p130Cas has been shown to induce apoptosis (Law et al., 2000; Wei et al., 2004). FAK, in part, regulates cell survival through binding to p130Cas and/or HEF1 - either sequestering them or altering their interactions with downstream effector proteins. 
Constitutively hyperphosphorylated forms of p130Cas have been detected in lung adenocarcinoma cells, perhaps permitting these cells to evade anoikis (Wei et al., 2002).

Src phosphorylation of p130cas allows it to interact with the adaptor molecule CRK through the SH2 domain. Interestingly, the related CRKII protein is required for mitochondrial cytochrome $\mathrm{c}$ release and caspase activation in Xenopus extracts (Smith et al., 2002). CRKII was found to interact with the tyrosine kinase WEE1, an inhibitor of cdc2/cyclinB activity, in the nucleus. WEE1 protein was also critical for apoptosis induction in this system, as was the interaction of CRKII with WEE1, but not the kinase activity of WEE1 itself. The second SH3 domain of CRKII interacts with the nuclear export protein CRM1, and mutation of this domain enhances the apoptotic effect of the CRKII protein, presumably by causing its retention in the nucleus. These results indicate that FAK might promote survival, in part, by sequestering CRK in the cytoplasm, thereby preventing its pro-apoptotic, WEE1-dependent function.

Cas and Crk may also play important roles in bypassing anoikis in normal epithelial cells during cell migration processes, such as those involved in wound healing. Factors that promote cell migration, such as EGF and hepatocyte growth factor (HGF), confer resistance to anoikis (Miller et al., 2003). Cos-7 fibroblasts embedded in collagen gels were found to undergo anoikis unless migration stimulated EGF or insulin. However, growth factor independent survival in this system was conferred by cotransfection of both CAS and CRK. Overexpression of CRK or hyperphosphorylation of $\mathrm{p} 130$ cas has been correlated with aggressiveness of lung adenocarcinoma. So CRK, in addition to its proapoptotic behavior described above, might mediate cell survival during cell migration. 
CAS, CRK or other cell migration-related molecules might make useful therapeutic targets for overcoming anoikis resistance or preventing metastasis.

Rac Signaling in survival pathways: Cell motility generally correlates with survival pathway and pathways affecting these involve Rac family of GTPases. Rac is critical for actin polymerization at the leading edge of migrating tumor cells (supports lamellopodia formation for movement) as well as activation of PAKs, which in turn contribute to ERK activation (Burridge and Wennerberg, 2004).

The small GTP-binding GTPases superfamily exist in cells as either the inactive guanosine diphosphate (GDP) bound form or the active GTP-bound form. The GTPbound form in turn is regulated positively by nucleotide exchange factors or negatively by GTPase activating protein. The GTPases such as Rac1, Cdc42, and nucleotide exchange factor Tiam1 have been shown to confer resistance to anoikis. Rac is activated either by integrin signaling following cell-matrix adhesion or by stimulation by motility factors. FAK also contributes to these pathways. Rac activation results in protecting cells through activation of ERK and Akt (Coniglio et al., 2001; Zahir et al., 2003). In a different context, alpha6beta4 intergrin mediated cell survival, Rac protected cells through activation of NFkB pathway (Zahir et al., 2003).

Rac is a known activator for PAK, supporting various pathways of cell survival. PAK1 phosphorylates Bad, promoting the release of Bcl-2 and activation of anti-apoptotic pathways. PAK4 also phosphorylates Bad and has been shown to promote anchorage independent growth (Gnesutta et al., 2001; Qu et al., 2001; Schurmann et al., 2000).

Non-integrin cell adhesion signaling in cell survival: Various non-integrin cell adhesion molecules have been shown to affect anoikis sensitivity and variably expressed 
during tumor progression and metastasis. These molecules include hemophilic cell adhesion molecules such as E-cadherin, PECAM, CEA, and CEACAM, hyaluronan receptor CD44 and neurotrophic factor binding receptor with tyrosine kinase activity, TrkB etc.

E-cadherin: During the early characterization of anoikis in MDCK cells, cell-cell interaction was reported to promote anoikis sensitivity whereas cells in sparse culture had dramatically reduced anoikis sensitivity (Frisch and Francis, 1994). This observation suggested that the cell-cell interactions sensitized cells to anoikis whereas their breakdown might contribute to loss of epithelial polarity and development of anoikis resistance. This observation has been supported by various experimental evidences that either loss of E-cadherin and/or cell polarity can make cells resistant to anoikis and promote tumor progression. E-cadherin is a cell adhesion molecule, which is required for maintenance of epithelial cell integrity by homophilic cell-cell interactions and formation of adherens junctions. E-cadherin controls the formation of catenin complexes to link actin and microtubule cytoskeleton (Perez-Moreno et al., 2003). In cancer, E-cadherin is frequently lost either through genetic or epigenetic mechanisms and promotes epithelialmesenchymal-transition in numerous malignancies. The targeted knockdown of Ecadherin gene in mouse mammary tumor model or the stable knockdown of E-cadherin in mammary epithelial cells promoted metastasis (Derksen et al., 2006; Onder et al., 2008) In later case, loss of E-cadherin results in "classic" EMT phenotype compared to earlier case suggesting the effect of cell type and epi (genetic) constitution. Incidentally, both of these cells were resistant to anoikis, further confirming that the presence of E-cadherin and resulting adherens junction are required for anoikis response in epithelial cells. In 
epithelial MDCK cells, $\mathrm{Ca}^{2+}$ dependent cell aggregation enhanced anoikis and surprisingly, the apoptotic cells in suspension cultures were mainly in single cells (Emoto, 2008). In enterocytes, early loss of E-cadherin in suspended cells from adherens junctions was required for onset of anoikis (Fouquet et al., 2004). The E-cadherin dismantling in enterocytes was a result of EGFR activation (Lugo-Martinez et al., 2009). The universality of anoikis mechanism in enterocytes is yet to be confirmed. The mammary epithelial cells, MDCK, and enterocytes anoikis data suggest that E-cadherin is required for sensitizing epithelial cells for anoikis and during suspension, apoptotic cells get free from E-cadherin mediated cell-cell aggregation. Alternatively, E-cadherin mediated cell aggregates leads to intracellular signaling, which in turn protect cells from anoikis as reported in normal tubular cells and squamous cell carcinoma (Zhang et al., 2004; Zhang et al., 2008). Apart from controlling adherens junction formation, E-cadherin also sequesters beta-catenin in cytoplasm and suppresses pro-oncogenic wnt signaling pathway. Loss of E-cadherin also reprograms cells for EMT by over-expressing EMT genes such as Twist and ZEB1. Interestingly, loss of E-cadherin mediated anoikis resistance can be partially reversed by downregulation of either Beta-catenin or Twist, which also suppressed the lung metastasis (Onder et al., 2008). Re-expression of Ecadherin can restore apoptosis sensitivity in EMT cells by sequestering beta-catenin in cytoplasm and suppressing its pro-tumorigenic transcriptional activity (Stockinger et al., 2001).

CD44: CD44 is an extracellular and cell-surface-associated glycosaminoglycan, which is a receptor for hyaluronan that is overproduced by many tumor types (Knudson, 1996). CD44's cytoplasmic domain is linked to the ezrin-radixin-moesin and ankyrin 
proteins that in turn bridge the actin cytoskeleton to the membrane (Ponta et al., 2003; Toole, 2004). It acts as a co-receptor for c-ErbB1-3, c-Met and other oncogenic receptors. Certain CD44 splice variants are overexpressed in various tumor types such as colorectal carcinoma and mammary carcinoma. The disruption of CD44-mediated cell adhesion to hyaluronan by the use of soluble, truncated CD44 receptors induces apoptosis in mammary carcinoma cells (Yu et al., 1997). Selection of human mammary tumor cells for those with the highest metastatic potential yielded a subpopulation of cells that overexpressed CD44, underscoring the functional importance of this protein in metastasis (Al-Hajj et al., 2003). CD44 variants exert a powerful cell survival effect with respect to various apoptotic stimuli, including DNA damaging agents and anti-integrin antibodies (Bates et al., 2001; Bates et al., 1998). Recently, CD44 has established as cancer stem cell marker and shown to overexpress in highly anoikis resistant cells which have undergone epithelial-mesenchymal-transition (Mani et al., 2008). The exact mechanism of CD44 in promoting cancer stem cells is unknown.

PECAM-1: PECAM-1 is a $130 \mathrm{kDa}$ immunoglobulin-domain protein that mediates homophilic cell adhesion at the surface of leukocytes, platelets and endothelial cells; it is involved in transendothelial migration of leukocytes and angiogenesis (Newman, 1997). Interaction between PECAM-1 on apposing cells has been shown to protect against a diversity of apoptotic stimuli, including serum-deprivation, gamma-irradiation, UV irradiation and staurosporine (Gao et al., 2003). Interestingly, transfected PECAM-1 protected 293 human embryonic kidney cells from apoptosis induced by BAX expression and did not inhibit the translocation of BAX from the cytosol to the mitochondria, but inhibited the cytochrome $\mathrm{c}$ release. Since BAX is important for the induction of apoptosis 
and therefore anoikis, it will be important to learn more about the anti-apoptotic mechanisms of PECAM-1, which have also been shown to involve interaction with the protein tyrosine phosphatase SHP-2 (Gilmore et al., 2000). Cell aggregations have been reported to suppress anoikis and promote survival. The suspended cultures of high cell density cultures promoted PECAM tyrosine phosphorylation. The cell survival depended on a novel PECAM interacting partner Pyk2 (Proline-rich tyrosine kinase 2), a member of FAK family member known for its role in adhesion and role in cytoskeletal structure regulation (Zhang et al.).

CEA and CEACAM: Carcinoembryonic antigen (CEA) and its relative, carcinoembryonic antigen-related cell adhesion molecule-6 (CEACAM-6), are membranebound, GPI-linked, immunoglobulin superfamily proteins that are overexpressed by a number of different carcinoma cell types including colon, breast, and lung etc. Overexpressed CEA has been shown to contribute to the anoikis-resistance of colon carcinoma cells (llantzis et al., 2002). Recently, the siRNA-mediated reduction of CEACAM-6 levels in pancreatic carcinoma cell lines was shown to promote anoikis and to inhibit metastasis of tumor cells in a nude mouse orthotopic xenograft model. The mechanism of anoikis-resistance by overexpressed CEA or CEACAM was considered by the involvement of Src-family kinases, modulation of a5b1 integrin function, or steric interference with the assembly of cell-cell junctional complexes. Recent studies have indicated that CEA interacts and suppresses pro-apoptotic activity of death receptor TRAIL-R1 (DR5) and its caspase-8 activation pathway in colorectal cancer (Samara et al., 2007). Another report using the overexpression of either CEA and/or a chimeric protein consisting of the NCAM extracellular domain attached to the CEA-GPI anchor 
activated PI3K/AKT pathway and inhibited caspase-9 activation, suggesting the involvement of intrinsic pathway (Camacho-Leal and Stanners, 2008). The universality of these studies is yet to be verified.

TrkB: The anti-apoptotic activity of the receptor tyrosine kinase TrkB, which binds the neurotrophin brain-derived neurotrophic factor (BDNF), has been well studied for many years by developmental neuroscientists (Davey and Davies, 1998; Jaboin et al., 2002). TrkB however is expressed by a variety of other cell types and its overexpression has been documented in pancreatic carcinoma, prostate carcinoma, Wilms tumor and neuroblastoma. TrkB was identified as an anoikis suppressor in a screen for genes that conferred epithelial cells with the ability to survive in suspension. TrkB expression did not however suppress serum withdrawal-induced apoptosis, indicating its specific activity in inhibiting apoptosis only under certain conditions. Its overexpression also caused several curious effects, including diminished cell-cell interactions of cells on two-dimensional culture, the formation of cell spheroids in suspended cell populations and Akt activation. The activated Akt has previously been shown to be involved in suppressing anoikis. Overexpressed TrkB also induced metastatic behavior of rat intestinal cells (Douma et al., 2004). The structural and functional characterization of TrkB indicated its kinase domain and not the extracellular adhesion motifs promoted anoikis resistance, oncogenicity and metastasis (Geiger and Peeper, 2007). The overexpression of TrkB in presence of its ligand BDNF, upregulated Twist via MAP Kinase dependent signaling pathway. Twist, in turn upregulated Snail followed by E-cadherin repression resulting in anoikis resistance and metastasis (Smit et al., 2009). Several inhibitors targeting TrkA or TrkB are being developed for cancer therapeutics. Few of the Trk inhibitors reaching clinical trial were 
CEP-751, CEP-701 (Cephalon) and AZ-23 (Astrazeneca). CEP-751 and CEP-701 suppressed tumor progression in xenograft models of prostate, pancreatic, neuroblastoma and medulloblastoma tumors. At present, CEP-701 is in phase 2 studies. There are no activating mutations of TrkB reported for the cause of tumor, so these inhibitors could be used as chemotherapeutic adjuvant in conjunction with standard chemotherapeutic agents (Thiele et al., 2009).

Nuclear Factors: Anoikis is a very immediate process. The translocation of Bax to mitochondria or caspase activation occurs within minutes of cell-extracellular matrix dissociation. Transcription factors may not contribute to the process of anoikis response. It is conceivable that transcription factors can reprogram cells for appropriate response during cell-extracellular matrix detachment. The stable expression of adenovirus E1a reprogrammed various human tumor cells to assume epithelial characteristics and sensitized them for anoikis (Frisch and Francis, 1994). Similarly various EMT inducing genes such as Twist, Snail, Slug, and ZEB1 etc. reprogram cells to lose epithelial characteristics and develop mesenchymal properties. The cells with mesenchymal properties are resistant to anoikis. The response to anoikis sensitivity could be programmed by regulation of epithelial and apoptotic genes.

One of the transcription co-repressor known as carboxy terminal binding protein (CtBP), known to co-operate with various repressors such as Zeb1 and repress various pro-apoptotic and epithelial genes such as PERP (p53-effector related to pmp-22), p21, Bax, and Noxa (Chinnadurai, 2009). The mesenchymal epithelial transition ability of E1a is partially through inactivation of CtBP (Grooteclaes and Frisch, 2000). In mouse embryo fibroblasts or human tumor cells, CtBP inactivation initiates a mesenchymal to epithelial 
transition, which upregulates epithelial specific genes such as E-cadherin. The cell types become dramatically more sensitive to anoikis. Recently, ZEB1 mediated metastasis by inhibiting stemness microRNA-203 and other microRNA (miR-200) family members and affecting expression of stemness gene such as polycomb repressor Bmi1 (Wellner et al., 2009). Targeting EMT genes and inactivating them either genetically or pharmacologically could reprogram cells for epithelial phenotype and can be used as an approach for cancer therapeutics.

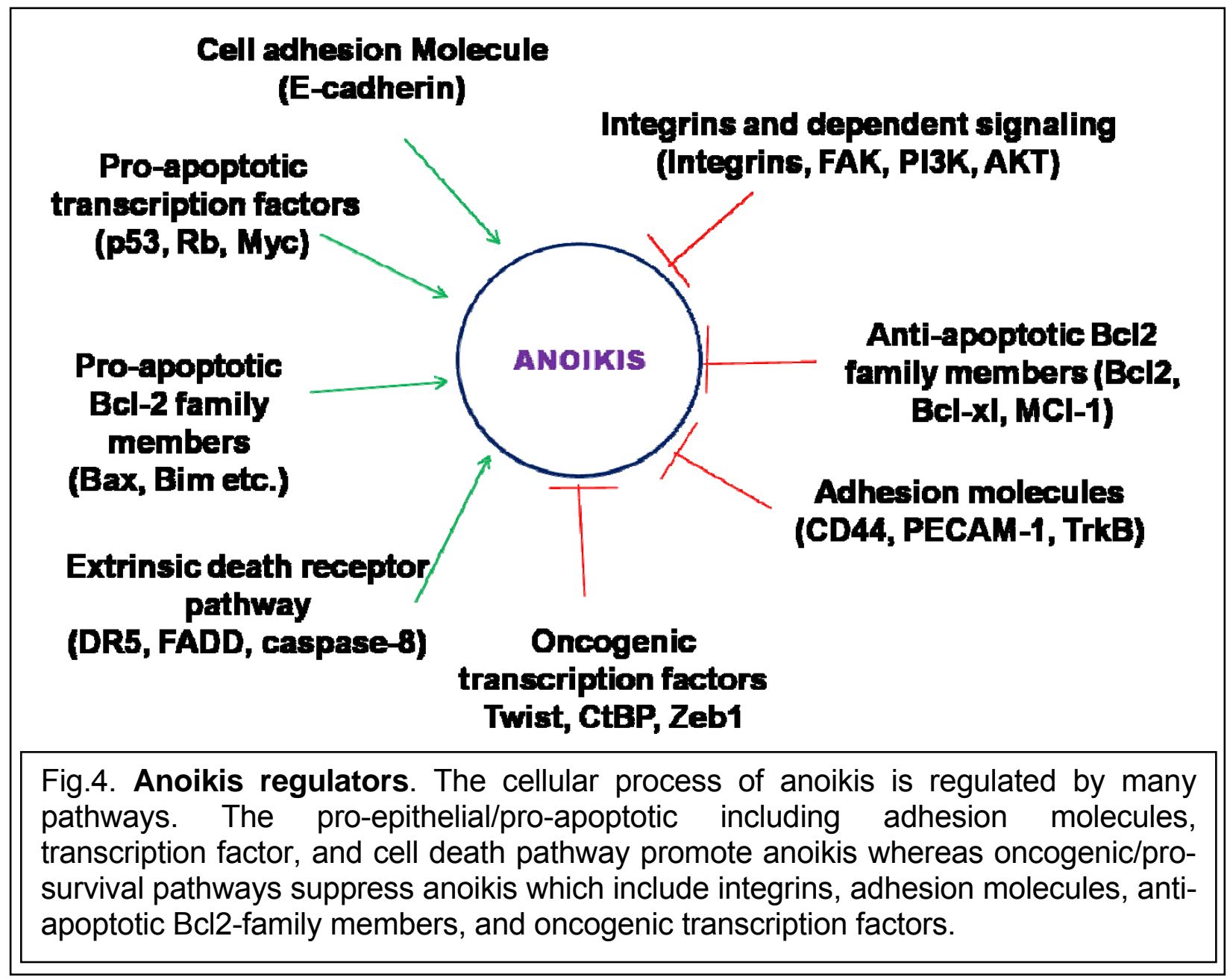

The very general overview has been summarized in fig.4. There are various other important advancements in the field of anoikis which may have remained overlooked in 
this review. Understanding cellular regulations for anoikis and targeting individual pathways could be used as an approach for cancer therapeutics.

\section{ANKYRIN}

Ankyrins are a family of adaptor proteins that link the actin cytoskeleton to membranes through interaction with spectrin and integral membrane proteins. They are ubiquitously expressed intracellular proteins evolved to perform specialized functions in metazoans and multicellular organisms, as they are absent in yeast and plants (Arabidopsis thaliana and Zea mays etc.) though present in Drosophila melanogaster (Dank1 and Dank2) and Caenorhabditis elegans (unc44) with high level of homology with their vertebrate orthologues (Mohler et al., 2002; Rubtsov and Lopina, 2000, Mohler, $2002 \# 164)$.

Ankyrins are encoded by three different genes in vertebrates and each contains multiple splice variants. The ankyrin prototype ankyrin-R named for its restricted expression in erythrocytes but also expressed in a subset of neurons, and striated muscles, is encoded by Ank1 on chromosome 8p11. Ankyrin-B, known for its broad expression, was first characterized in brain. Ankyrin-B is encoded by Ank2 gene on human chromosome $4 q 25-27$. The third ankyrin isoform ankyrin-G, named for its giant size and general expression pattern is encoded by Ank3 on human chromosome 10q21. This is also considered as epithelial ankyrin-G for its role in epithelial cell maintenance (Mohler et al., 2002).

Ankyrin domains: All of the three isoforms of ankyrin have common domain structures. Ankyrins have three major common domains namely membrane binding domain (MBD) which contains ankyrin repeats (AR), spectrin binding domain (SBD), and 
the ZU5-UPA-DD supermodule (fig.5). The diversity in different ankyrin gene products is because of the variation in regulatory regions at the extreme c-terminus. The exact function of the regulatory region is currently unknown. The spectrin binding domain has been characterized as the function of its ZU5 domain and subsequent 55 amino acids (Mohler et al., 2004). Further characterization based on sequence homology between ZU5 and death domain containing proteins suggested the presence of a supermodule containing another ZU5 (ZU5-B), UPA (named for being conserved in unc5, PIDD and ankyrin) and the death domain (Wang et al., 2009).

The membrane binding domain (MBD), known for the presence of various ankyrin repeats (AR), is named for interactions with multiple membrane proteins. The ankyrin MBD contains 24 ankyrin repeats and helps assemble the multiprotein complexes at specific cellular sites. This domain is involved in binding to ion channels/pumps such as anion exchanger isoforms (AE1, AE2, AE3), the $\mathrm{Na}^{+}-\mathrm{K}^{+}$-ATPase, the voltage-dependent $\mathrm{Na}^{+}$-channel and $\mathrm{Na}^{+} / \mathrm{Ca}^{+}$exchanger; IP3 receptor and ryanodine receptor, $\mathrm{Ca}^{2+}$ release channels, cell adhesion molecules (CAMs) including CD44, L1 CAM family (L1/neurofascin/NrCAM/CHL1/NgCAM/LAD-1/neuroglian), cytoplasmic proteins such as tubulin and clathrin, and the guanine nucleotide exchange factor (GEF) Tiam1. These interactions regulate a variety of cell processes including ion homeostasis, adhesion functions, and protein trafficking (Mohler et al., 2002). Each ankyrin repeat (AR) is 33 amino acids tandem repeats, forms protein-protein interaction motifs, and found in a large number of functionally diverse eukaryote proteins (rarely in prokaryotes), and the presence is hypothesized because of horizontal gene transfer. Ankyrin repeats (AR) are structural rather than functional motifs and are found in proteins involved in transcriptional 
regulation such as GABP beta and NF-kappaB inhibitor IkappaB, cell-cycle regulators tankyrase and p53-binding protein (53BP2), cytoskeleton (ankyrins), ion transporters (TRP family of ion channels) and signal transducers. Each of the ankyrin repeats fold into a helix-loop-helix structure with a beta hairpin/loop region projecting out from the helices at a $90^{\circ}$ angle. The repeats stack together to form an L-shaped structure. The crystal structure of ankyrin repeats suggested that they form spiral stacks, and the spectrin binding domain fragment interacts as an extended strand. Based on in vitro binding studies and the structural model, ion transporters interacts with large central cavity formed by spiral ankyrin repeats whereas clathrin and cell adhesion molecules associate through the specific regions outside this cavity (Michaely et al., 2002). The antiparallel alpha helices are predicted to form superhelical spiral stacks, and atomic force microscopic measurement suggested the reversible linear elasticity extended in multiple stretches and exhibited nanospring like properties. The mechanical properties in turn are predicted to respond to mechanical strain and generate flexibility in membrane bilayer plane (Lee et al., 2006).

The central domain of ankyrin consists of spectrin binding domain (SBD) in all of the three ankyrin gene products. Ankyrin was first identified on the basis of spectrin binding in erythrocytes. Mutation in ankyrin-SBD leads to the development of several diseases in human and mice such as hereditary spherocytosis and reduced levels of axon initial segments. The interaction studies using yeast 2-hybrid identified the internal ZU5 domain (named for its presence in ZO1 and Unc5H1) along with 55 amino acids as the minimum spectrin binding domain. The ZU5 domain within spectrin binding domain is first among the two ZU5 domains and so, being referred to as ZU5-A. Site directed 
mutagenesis of DAR976AAA and A1000P within ZU5-A abolished the ankyrin-spectrin interaction (Mohler et al., 2004). This interaction has been verified biochemically and the interaction ratio between ZU5 domain and $14^{\text {th }} / 15^{\text {th }} \beta$-spectrin was found to be 1:1 (Ipsaro et al., 2008). Further structural characterization revealed that the $14^{\text {th }}$ spectrin repeat contains a conserved negatively charged patch which could account for its interaction with the positively charged ZU5-A domain. The ZU5-A domain contains a conserved beta core structure but lacks part of canonical ZU5 consensus sequence, which makes it structurally more appropriate for spectrin binding (Ipsaro et al., 2009).

The recently identified ZU5-UPA-DD supermodule function is currently not well understood. Part of this supermodule contains the death domain (DD) which has previously been characterized in the context of receptor mediated apoptotic pathways. The death domain was first identified in TNF family receptor (i.e. TNF receptor and Fas). The death domains are known to homodimerize and heterodimerize with other death domain proteins. The high affinity death domain proteins such as FADD, TRADD and RIP were identified using the yeast two-hybrid system, whereas low affinity death domain proteins such as nerve growth factor receptor (NGFR) and ankyrin were identified by sequence homology. There are two subtypes of death domains, subtype 1 includes Fas, TNFR1 (TNF Receptor1 or p55-R), FADD, ankyrin, RIP and TRADD whereas the subtype 2 includes DAP Kinase, NGFR, p100 and p105 members of Rel protein family, and myD88. All of the death domains are predicted to have five sequential alpha helices with exceptions of either of one missing in different types (Feinstein et al., 1995). Function of the ankyrin death domain (ankDD) is not well understood. Recently, the death domain of ankyrin was shown to interact with Fas (yeast 2-hybrid and co-immunoprecipitation in 
MDCK cells) and FADD (co-immunoprecipitation in MDCK cells) through their death domains. The site directed mutagenesis of conserved ankyrin-G R1496 abolished this interaction. In a mouse model of renal ischemia-reperfusion injury, the expression as well as interaction between ankyrin and Fas increased markedly. The MDCK cells stably expressing ankyrin-G, responded to Fas ligand induced apoptosis in MDCK cells. This was the first demonstration of ankyrin-G in regulation of cell death (Del Rio et al., 2004). The universality of this interaction and pathway is yet to be confirmed.

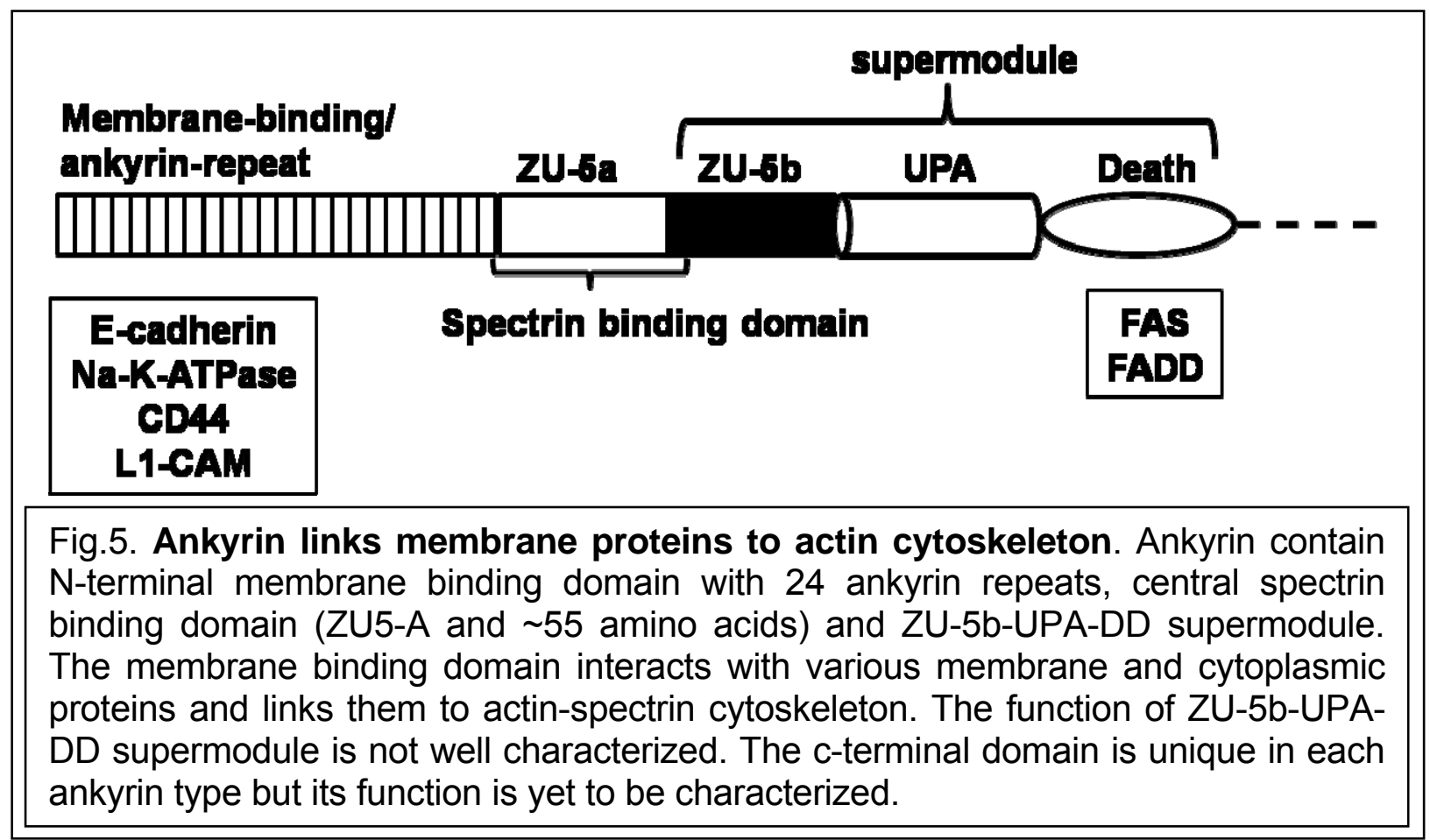

Ankyrin-G: Ankyrin-G was first characterized in brain tissues and exhibited extremely high molecular sizes (480 kDa and $270 \mathrm{kDa})$. Apart from the common ankyrin domain structures (i.e. MBD, SBD and ZU5-UPA-DD supermodule), these variants contain a unique serine and threonine rich domain of more than $200 \mathrm{kDa}$, restricting ankyrin-G at axonal proximal segments (Kordeli et al., 1995). The ankyrin-G null (-/-) 
mouse neurons failed to restrict the voltage-gated sodium channels ( $\mathrm{NaCh}$ ) and neurofascin ( $\mathrm{L} 1$ family member of adhesion molecule) at their axon proximal segments, suggesting the role of ankyrin-G in specialized membrane domain assembly (Zhang and Bennett, 1998). The membrane binding domain of ankyrin-G interacted with neurofascin through conserved amino acids ser56-tyr81, and mutagenesis of FIGQY tyrosine $(Y 81 H / A / E)$ greatly impaired the interaction. The mutation in equivalent tyrosine of $L 1$ has been linked to certain cases of mental retardation, suggesting the similar role of ankyrin interaction modules of neurofascin (Zhang et al., 1998). The cerebellum specific ankyrinG knockout mouse exhibited progressive ataxia at the beginning of postnatal day P16. These mice also lost the Purkinje neurons, highlighting the importance of ankyrin-G in the assembly of normal brain tissues (Zhou et al., 1998). Recently, ankyrin-G has been shown to interact with beta1 subunit of cyclic nucleotide-gated channel (CNG beta1) in the plasma membrane of photosensitive outer segments (OS) of rod photoreceptors. Ankyrin-G was critical for CNG beta1 targeting to the rod outer segments. The ankyrin-G knockdown in neonatal mouse retina markedly reduced CNG channels expression. The ankyrin interaction was attributed to the 28 amino acids at c-terminus of CNG beta1, the deletion previously reported in the retinitis pigmentosa patients. This observation highlights the critical role of ankyrin-G in CNG beta1 membrane targeting and its physiological relevance in photoreceptors function (Kizhatil et al., 2009).

Ankyrin- $G$ has been reported to be critical for epithelial membrane biogenesis. The ankyrin-G knockdown in human bronchial epithelial cells by small interfering RNA resulted in the loss of lateral plasma membrane in interphase cells. The cells also lost the post mitosis membrane biogenesis, although the apical/basal plasma membranes and cell 
polarity remained unaffected. Additionally, the localization of ZO1 in tight junction and Ecadherin/beta catenin in adherence junctions remained unaffected, whereas E-cadherin and $\mathrm{Na}^{+}-\mathrm{K}^{+}-\mathrm{ATPase}$ in lateral membrane were severely affected. The membrane biogenesis could be rescued by the re-expression of rat ankyrin-G (siRNA resistant). However, chimeric ankyrin-G containing either of ankyrin-B domains (MBD or SBD or DD) failed to restore ankyrin-G dependent membrane biogenesis, suggesting the pleiotropic requirement of all ankyrin-G domains in membrane biogenesis (Kizhatil and Bennett, 2004). Ankyrin-G interacting ion pump $\mathrm{Na}^{+}-\mathrm{K}^{+}-\mathrm{ATPase}$ has been shown to lose the cell polarity following ischemic injury. ATP depletion resulted in duration dependent mislocalization of ankyrin-G and $\mathrm{Na}^{+}-\mathrm{K}^{+}-\mathrm{ATPase}$ without affecting their interaction in Madin-Darby canine kidney (MDCK) cells. Further characterization revealed that the ATP depletion affected ankyrin-G-spectrin interaction, which in turn correlated with ankyrin-G hyperphosphorylation (serine/threonine and tyrosine). This observation adds further complexity on ankyrin-G mediated cytoskeleton re-organization, involving posttranslational modification (Woroniecki et al., 2003). Incidentally, ankyrin phosphorylation has previously been reported to affect its interaction with spectrin which depended upon serine/threonine phosphorylation by casein kinase 2 (CK2) (Ghosh and Cox, 2001; Ghosh, 2002). The post-translational modification of ankyrin-G would allow rapid membrane and cytoskeleton reorganization depending on various physiological stimuli.

Ankyrin-G is a known interacting partner of cell adhesion molecule E-cadherin, first shown in MDCK cells (Nelson et al., 1990). Recently, the physiological significance of ankyrin-G and E-cadherin interaction was characterized. Ankyrin-G interacted and accumulated E-cadherin at lateral membrane and early embryos. The beta2 spectrin and 
ankyrin-G were required for E-cadherin lateral membrane targeting in microtubuledependent manner. The multi-protein complex including spectrin-ankyrin-E-cadherin in turn co-ordinated the membrane assembly with extracellular interactions at cell-cell contact site (Kizhatil et al., 2007). E-cadherin is a critical adhesion molecule known for its requirement in epithelial cell maintenance. Loss of E-cadherin is the preliminary step in losing cell polarity and acquiring epithelial-mesenchymal-transition. Based on E-cadherin interaction, ankyrin-G is predicted to regulate various cellular processes during epithelial maintenance (in presence of E-cadherin) and EMT, tumor progression and metastasis (in absence or nonfunctional state of E-cadherin).

Role of Ankyrin-G in Cancer: The role of ankyrin-G is not well understood in cancer. The interactions with hyaluronan receptor (CD44) and nucleotide exchange factor (Tiam1) have highlighted a few of the roles of ankyrin in tumor progression. Especially,the interactions between CD44 and ankyrin have been reported in various cell types including T-cells, endothelial cells, breast, ovary and prostate cancer cells (Bourguignon et al., 1998; Bourguignon et al., 1992; Kalomiris and Bourguignon, 1989; Welsh et al., 1995; Zhu and Bourguignon, 2000). The 15 amino acid cytoplasmic region of CD44 was sufficient for ankyrin interaction. The hyaluronic acid (HA) treatment induced CD44ankyrin interaction and enhanced cell adhesion (Lokeshwar et al., 1994). Variable expression of CD44 among prostate cancer cell lines was compared for invasiveness and tumorigenesis. The CD44 negative LNCaP cells exhibited a less malignant phenotype compared to aggressive tumorigenesis by PPC-1 and ALVA-31 (expressing multiple CD44 variants), which in turn correlated with HA-dependent ankyrin localization and cell adhesion (Welsh et al., 1995). The over-expression of CD44 in LNCaP cells promoted c- 
Src dependent anchorage independent growth, which in turn was dependent on the 15-aa ankyrin binding sequence (Zhu and Bourguignon, 1998). The ankyrin-CD44 interaction has also been reported to promote breast cell tumorogenesis. The highly metastatic breast cancer cells, Met1 (derived for transgenic mice expressing polyoma virus middle T oncogene), expressed CD44v3,8-10 variant with intact ankyrin interaction as reported with other CD44 variant and formed matrix metalloprotease-9 (MMP-9) dependent invadopodia (Bourguignon et al., 1998). Further characterization demonstrated the physical interaction between CD44v3,8-10 and RhoA GTPase and activated Rho Kinase (ROK). The activated ROK phosphorylates the cytoplasmic domain of CD44v3,8-10, enhances its interaction with ankyrin, regulates membrane ruffling and cell projections with increased membrane motility and tumor cell migration (Bourguignon et al., 1999). In metastatic breast tumor cells MDA-MB-231, CD44 interacted with TGF beta receptor. The HA-treatment activated the serine-threonine kinase activity of TGF beta receptor, which in turn phosphorylates CD44 to enhance interaction with ankyrin. The increased interaction correlated with increased cell migration and other tumor related activities (Bourguignon et al., 2002). HA-treatment also enhanced CD44 and nanog interaction in breast tumor cells (MCF-7 cells) and ovarian tumor cells (SK-OV-3 cells). Nanog formed a complex with Stat-3 and activated the transcription of Stat-3 target genes including multidrug transporter, MDR1. The HA-CD44 interaction induced ankyrin interaction with MDR1 to increase the efflux of chemotherapeutic drugs. The report of the breast and ovary cancer cells displaying stem cell properties and novel pathways to develop chemotherapeutic resistance can be targeted for cancer therapeutic pathways (Bourguignon et al., 2008). 
The nucleotide exchange factor Tiam1 interacts with ankyrin in breast tumor cells. The Tiam1-ankyrin interaction in membrane projections activated Rac1, which in turn increased cell invasion and migration (Bourguignon et al., 2000). The CD44 and Tiam1 mediated oncogenic signaling and tumor progression depended partially on ankyrin-G interaction and cytoskeletal re-organization.

Nevertheless, there are evidences which would support ankyrin-G anti-tumorigenic activity. The study using mouse/human comparative translational genomics approach to identify a death-from-cancer signature predicting therapy failure identified an 11-gene signature. Ankyrin-G was one of the 3 down-regulated genes, suggesting its inverse correlation with cancer progression (Glinsky et al., 2005). In another study, TGF-beta mediated human hepatocellular carcinoma model demonstrated downregulation of ankyrin-G (Gotzmann et al., 2006). The tumor suppressor hepatocyte nuclear factor 4 alpha (HNF4 alpha), which is downregulated during renal cell carcinoma, upregulates ankyrin-G (Grigo et al., 2008). In an unrelated study to characterize the gene expression during premature aging disease Hutchinson-Gilford progeria syndrome (HGPS), ankyrin$\mathrm{G}$ was identified as an overexpressed (>100 fold) gene which correlated with increased apoptosis in patient fibroblasts (Wang et al., 2006). All these studies indicate that ankyrin$\mathrm{G}$ as either anti-tumorogenic and/or pro-apoptotic genes (fig.6). The discrepancy between pro-tumorogenic and anti-tumorogenic/pro-apoptotic ankyrin-G function may be result of the cell context, post-translational modification, function of variable splice variants and multiple interaction complexes with different outcomes. For example, an isoform of ankyrin-G that lacks the membrane binding domain (ANK-105) and localizes to 
endosomes/lysosomes, interacts with p85 and plays a role in lysosome mediated PDGF receptor downregulation (Ignatiuk et al., 2006).

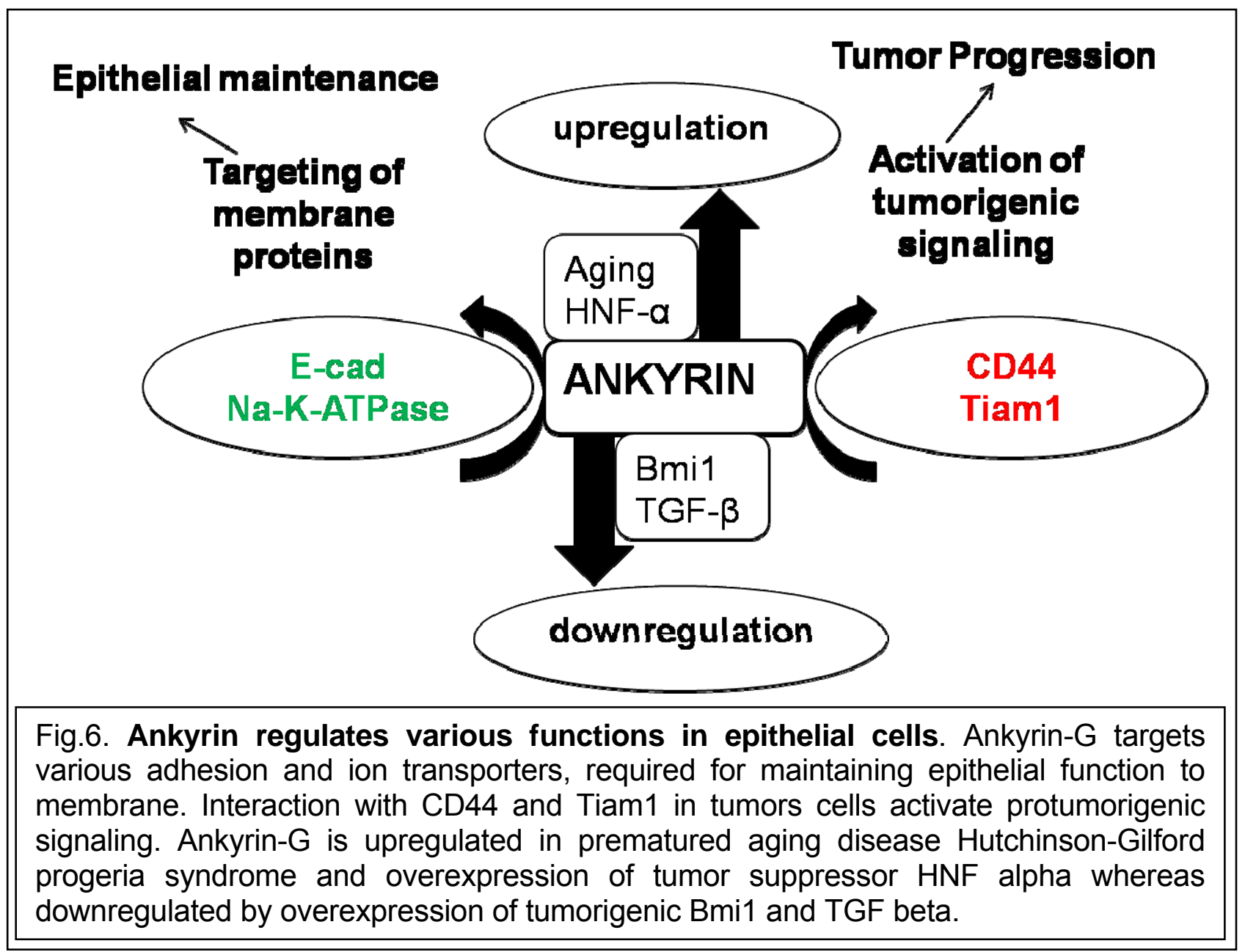

NRAGE

NRAGE (Neurotrophin Receptor-interacting mAGE protein), also known as MAGED1 and Dlxin1 (DIx5 interacting protein), is a member of the MAGE (Melanoma Antigenencoding GEne) family protein, named for its interaction with neurotrophin receptor ( $p 75)$. The MAGE family members are characterized by the presence of melanoma homology domain (MHD). The first reported MAGE family protein was identified as the peptides 
derived from proteins found in complex with melanoma patients major histocompatability complex 1 (MHC1), which could be identified by cytotoxic T lymphocytes in vitro (Pold et al., 1999).

The melanoma antigen (MAGE) family is comprised of two subfamilies, type I, which are expressed only in embryonic tissue and melanomas, and type II, expressed almost ubiquitously in adult tissues. The type I members are highly expressed in melanomas and are a popular focus of immunotherapy researches as potential tumor antigens for developing cancer vaccines. There are more than 50 members identified and share a highly conserved $\sim 190$ amino acid MAGE homology domain (Barker and Salehi, 2002; Chomez et al., 2001). NRAGE belongs to type II MAGE family protein which expresses in a variety of epithelial, neuronal and tumor tissues (Bertrand et al., 2004; Pold et al., 1999) and encodes by locus Xp11.21-p11.23 on chromosome X (Zhang et al., 2001).

NRAGE domain structure: NRAGE, identified for the presence of MHD, also contains few other domains such as $\mathrm{N}$-terminal domain, DNA polymerase homology domain (DNAPIII), WQXP repeat domain, and the C-terminal domain (fig.7). The function of N-terminal domain and DNAPIII domain are not characterized. The WQXP repeat domain contains 25 repeats of hexapeptide WQXXPXX, conserved in all species of MAGE-D1, but there is no homology otherwise in the existing database. The WQXP repeat domain interacts with a homeodomain box containing transcription factors Dlx5/Msx2 (Masuda et al., 2001). The NRAGE's MAGE homology domain interacts with various membrane and cytoplasmic proteins such as p75-NTR, Unc5H1, Ror2, Necdin, and Praja1 (Sasaki et al., 2005). The C-terminal domain of NRAGE interacts with Che-1, 
an apoptosis antagonizing transcription factor (AATF) (Passananti and Fanciulli, 2007). In summary, NRAGE interacts with three groups of proteins such as membrane receptors (p75-NTR, unc5H1, and Ror2), transcription factors (Dlx5/msx2 and Che-1), and cytoplasmic proteins (XIAP and Praja1).

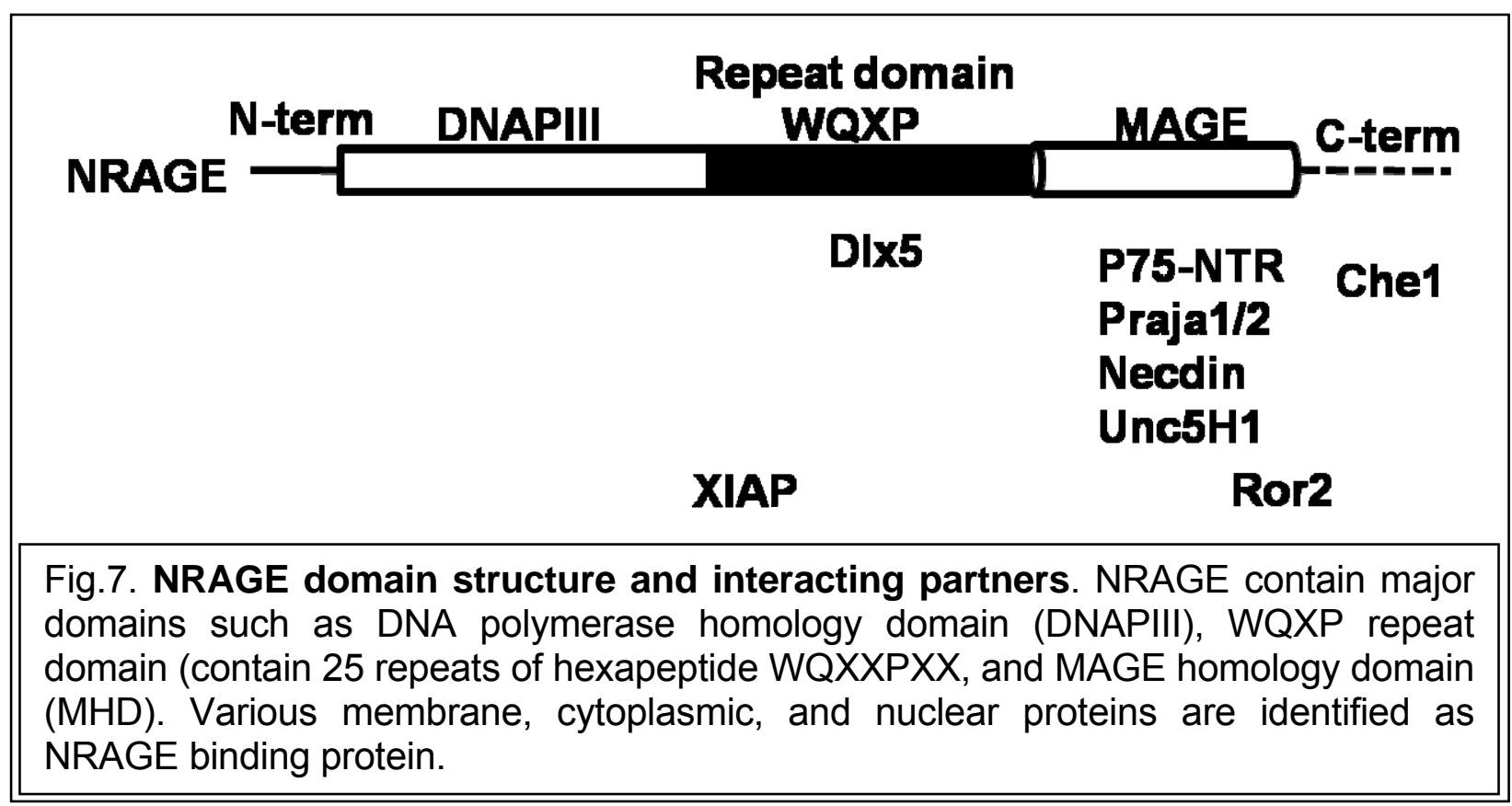

NRAGE interacting partners: NRAGE interacts with membrane, cytoplasmic and nuclear proteins (fig.7). The role of NRAGE in context of individual interactions is briefly summarized.

NRAGE was first identified as a p75-NTR interacting protein. The interaction increased upon NGF treatment, blocked the physical interaction between p75-NTR and trkA and enhanced apoptosis and cell cycle arrest in sympathetic neurons (Salehi et al., 2000). NRAGE was also identified as axon guidance netrin receptor, unc5H1 interacting protein. The interaction enhanced apoptosis in undifferentiated PC12 cells. The differentiated PC12 cells downregulated NRAGE and failed to respond to unc5H1 overexpression. The co-expression of NRAGE and unc5H1 restored the apoptosis 
sensitivity in differentiated PC12 cells (Williams et al., 2003). The NRAGE-mediated apoptosis was result of the recruitment of ubiquitin ligases to IAPs, causing their degradation, and by activating Jun-N-terminal Kinases (JNKs) to activate the mitochondrial dependent caspase pathway (Salehi et al., 2002). Consistent with this proapoptotic function, NRAGE knockout mice show a developmental defect in neuronal apoptosis in the brain (Bertrand et al., 2008).

NRAGE was identified as homeodomain protein Dlx5 interacting protein (so named Dlxin1) and the interaction in turn regulated the Dlx-5 dependent transcription activity (Masuda et al., 2001). NRAGE interaction with another MAGE family protein, Necdin, was shown to release the Msx1/2 (muscle segment homeobox 1/2) mediated transcriptional repression (Kuwajima et al., 2004). The NRAGE function is also regulated by its interaction with E3 ubiquitin ligase Praja1. NRAGE is hypothesized to link E3 ubiquitin ligases to its interacting partners such as Msx2/DIx5 and affecting their stability/transcriptional function (Sasaki et al., 2002). The NRAGE interaction with transcription factors has been characterized but their role in transcription regulation is still an open ongoing area of discussion.

Role of NRAGE in cancer: NRAGE is a type II MAGE family protein, expresses ubiquitously in all tissue types and interacts with various membrane and nuclear proteins. There are various studies performed to understand its role in cancer. The study performed in melanoma and pancreatic cancer demonstrated that the adenovirus mediated overexpression of NRAGE suppressed cellular motility and cancer motility possibility through regulating matrix metalloproteases-2 (MMP-2) activity (Chu et al., 2007). Similar observations were also made during the study in NRAGE overexpressing 
breast cancer cells, attributing the effect on p53, p21, and E-cadherin upregulation and beta catenin downregulation. Though the effect was only observed on one cell line, questioning the universality of this observation (Du et al., 2009). These observations have shown the pro-tumorogenic role of NRAGE in tumorogenicity.

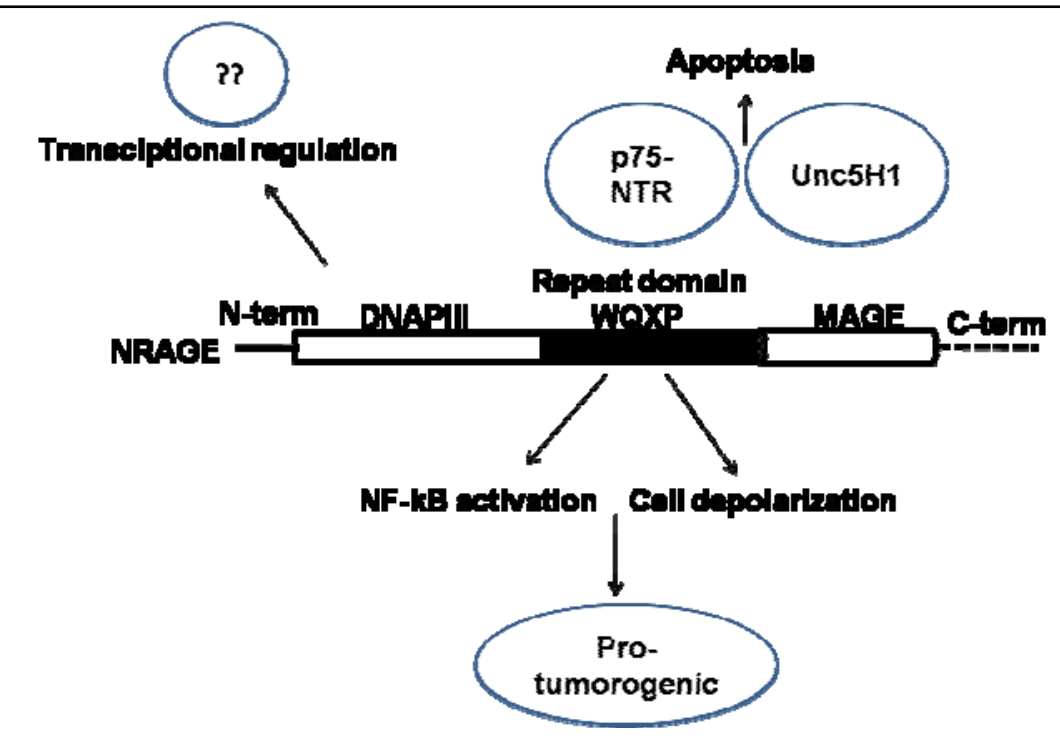

Fig.8. NRAGE shows pro-apoptotic and pro-tumorigenic function. NRAGE has been characterized as pro-apoptotic with reference to $\mathrm{p} 75$ and $\mathrm{Unc} 5 \mathrm{H} 1$ interaction whereas pro-tumorigenic with reference to NF-kB activation and cell depolarization activities. The NRAGE role in transcriptional regulation is not well characterized.

Nevertheless, there are other evidences suggesting the pro-tumorigenic role of NRAGE. The overexpression of NRAGE disrupts colocalization of E-cadherin/beta catenin and translocation of beta catenin to cytoplasm and nucleus in osteosarcoma cells U2OS. The loss of cell polarity/adherence junction and localization of beta catenin have been implicated in the pro-tumorigenic activity (Onder et al., 2008). Recently, NRAGE have been shown to activate NF-kB pathway in non-canonical bone morphogenic protein signaling pathway (Matluk et al.). NF-kB activation is well characterized for proinflammatory and pro-tumorigenic pathways. Few other reports have indicated NRAGE 
over-expression in various cancer patients including lung, kidney and head/neck (Bhattacharjee et al., 2001; Boer et al., 2001; Ginos et al., 2004). The effect on cell polarity, NF-kB activation and overexpression in various cancer types could account for NRAGE's pro-tumorigenic activity (fig.8).

There are no mechanistic details for role of NRAGE role in tumor progression/cancer. The other proteins related to NRAGE can give some insight about its possible role in cancer. One of the related proteins MAGE-A3 supports melanoma cell survival, and overexpression in lung cancer correlates with the poor prognosis (Groeper et al., 2007; Yang et al., 2007). The NRAGE orthologue trophinin/MAGE-D3 is overexpressed in colorectal carcinoma, promoting tumor metastasis (Harada et al., 2007). The function of NRAGE is pro-apoptotic with respect to receptors such as p75-NTR and Unc5H1 but the possible tumorigenic role in anoikis is not yet tested. The phosphotyrosine enriched NRAGE has been identified in immunoaffinity profiling of phosphorylated tyrosine in cancer cell lines, suggesting its possible post-translational regulation during tumor progression (Rush et al., 2005).

TBX2

TBX2 belongs to T-Box (TBX) family of transcription factors, known to regulate various cellular processes such as embryonic development, cell cycle progression, senescence and apoptosis (Abrahams et al.). Various reports have indicated the TBX2 overexpression in variety of cancer types including melanoma, small cell lung carcinoma, breast, pancreatic, liver, and bladder cancers. Recently, TBX2 has been reported to promote anchorage independent growth in p53 negative SW13 adenocarcinoma cell line 
and mouse embryo fibroblast lacking retinoblastoma $(\mathrm{pRb})$ and related pocket proteins (p107 and p130) (Ismail and Bateman, 2009; Vormer et al., 2008).

TBX2 has traditionally been characterized as a transcription repressor. The murine TBX2 study suggested its possible function in transcription activation. The transcription activation domain was identified as part of the $\mathrm{N}$-terminal domain (Paxton et al., 2002). There are various targets of TBX2 including tumor suppressor p14-ARF, p21, and NDRG1 (N-myc downregulated gene 1) (Jacobs et al., 2000; Prince et al., 2004; Redmond et al.). In contrast, TBX2 has been shown to upregulate hyaluronan synthase 2 (HAS2) and TGF beta2 as a result of the Bmp-Smad pathway (Shirai et al., 2009). The universality of these transcription regulations are yet to be tested. Few of the TBX2 targets such as P14-ARF and NDRG1 are briefly described here.

P19-ARF (P14-ARF in human) was identified as TBX2 target in a screen bypassing senescence. TBX2 was shown to attenuate E2F1, Myc or RAS-mediated induction of P19-ARF (Cdkn2a). P14-ARF (alternate reading frame) is encoded by INK4 locus, which also encodes p15 and p16. P14-ARF shares exon2 and exon3 with p16 gene but the reading frame is completely different. P14-ARF is one of the most frequently mutated genes in human carcinoma (Kamb et al., 1994). P14-ARF is involved in various signaling pathways such as p53, c-myc and CtBP and promotes apoptosis. The recently identified TBX2 target is NDRG1. TBX2 represses NDRG1 in an EGR-1 dependent mechanism, to promote proliferation in breast cancer cells (Redmond et al.). NDGR1 is previously reported for its function as metastasis suppressor, negatively correlated with tumor metastasis (Kovacevic and Richardson, 2006). Its role in anoikis and anchorage independence growth is yet to be characterized. 


\section{SUMMARY}

Anoikis is an important epithelial feature to maintain the cell homeostasis and suppress tumor metastasis. The process is supported by various cellular proteins such as transcription factors, extracellular proteins, adhesion molecules, cytoskeletal proteins, death receptors pathway proteins, Bcl2 family members (pro- and anti-apoptotic proteins) and various signaling pathways proteins. During EMT and tumor metastasis, cells develop various mechanisms that could change cell behavior and growth pattern. In this study, we are studying the role of a cytoskeletal protein ankyrin-G, MAGE family protein NRAGE, and a known embryonic and oncogenic transcription repressor TBX2 for their role in anoikis and anchorage independent growth. This is a very novel study implicating new partners for their role in anoikis and their possible regulations during EMT and tumor progression. 


\section{CHAPTER 2}

\section{MATERIALS AND METHODS}

Cell Culture: The Madrin Darby canine kidney (MDCK), MDA-MB-231, 293T and HT1080 cells were maintained in DME medium supplemented with $10 \%$ fetal bovine serum and $1 \mathrm{X}$ penicillin/streptomycin/glutamine. The non-small cell lung carcinoma cell line $\mathrm{H} 1299$ ( $\mathrm{NCl}-\mathrm{H} 1299)$ cells, breast cancer cells Au565 and BT474 were maintained in RPMI medium supplemented with $10 \%$ fetal bovine serum and $1 \mathrm{X}$ penicillin/streptomycin/glutamine. MCF-7 cells were maintained in DME medium supplemented with $10 \%$ fetal bovine serum, $1 \mathrm{X}$ penicillin/streptomycin/glutamine and 10 $\mu \mathrm{g} / \mathrm{ml}$ insulin. MCF10a cells, kindly provided by R. Pauley (Karmanos Cancer Center, Detroit, MI), were maintained in DME/F12 medium supplemented with $5 \%$ horse serum, 1X penicillin/streptomycin/glutamine, $10 \mu \mathrm{g} / \mathrm{ml}$ insulin, $10 \mathrm{ng} / \mathrm{ml}$ EGF, $0.5 \mu \mathrm{g} / \mathrm{ml}$ hydrocortisone and $0.1 \mu \mathrm{g} / \mathrm{ml}$ cholera toxin. HMLE, HMLE+shEcad and HMLE+Twist cells, kindly provided by the R. Weinberg lab (Whitehead Institute, Cambridge, MA) were maintained in the same medium but without cholera toxin (plus 20nM 4-hydroxytamoxifen for the Twist cells). I-HUVEC cells (Freedman and Folkman, 2004), kindly provided by the Folkman lab (Harvard Medical School, Boston, MA) were maintained in EGM-2 medium (Cambrex) plus $5 \%$ fetal bovine serum. Derivatives of MCF10a and HMLE cells were made by infection with recombinant retroviruses that were packaged by co-transfection into the cell line gp2+293 with the vector pAmpho (Clontech). The human NRAGE construct from Origene, containing MAGE-D1 transcript variant 2 (778 amino acids, accession number NM_006986), was used as a template for all NRAGE constructs. NRAGE was PCR-amplified with a forward primer that included a HA-tag sequence and 
Xhol site, and subcloned into the Xhol-Notl site of MSCV-IRES-puro. To construct NRAGE-NLS, the complete NRAGE coding sequence upstream of the stop codon was amplified with the same forward primer as above, and with a reverse primer containing an Nhel site. Separately, the triple NLS sequence from the vector pEYFP-NLS (Promega, Madison, WI) was PCR-amplified to produce an Nhel-Notl fragment. The NRAGE and NLS fragments were three-way ligated into the MSCV-IRES-puro vector. We observed that infection of constructs in this vector produced cell lines of variable anoikis-sensitivity following puromycin selection, even with the empty vector (data not shown). Therefore, the NRAGE and NRAGE-NLS sequences from these constructs were subcloned into the Xhol site of pMIG (Addgene, Cambridge, MA) by PCR. Following infection, cells were flow-sorted for GFP-positive cells, due to the GFP marker present in the retrovirus. Expression of NRAGE was found to be three-fold above the endogenous level by western blotting with NRAGE mAb clone 48 (BD Biosciences, Pharmigen), and uniform expression of NRAGE was verified by immunofluorescence using HA antibody HA.11 (Covance, Emeryville, CA). P19ARF-knockout and control wild-type mouse embryo fibroblasts (MEFs) were kindly provided by Ned Sharpless (University of North Carolina). These were immortalized with SV40 T-antigen using the retroviral producer cell line, ZipTer, and T-antigen expression was verified by western blotting.

Expression constructs: Ankyrin-FL construct was subcloned from pcDNA3-Flagrat-ankG190 (a kind gift from Devarajan Prasad) to pcDNA3.1 (-) His-myc. The ankdelZU5 and ank-deIDD were designed by ligating n-terminal and c-terminal PCR products in pcDNA3.1 (-) myc-His (Invitrogen, Carlsbad, CA, USA). Ankyrin-MBD, ankyrin-DD and ankyrin-ZU5 were made by amplifying individual domains using pcDNA-ankG190 as 
template and sub-cloning to pcDNA3.1 (-) myc-His. GST-ankDD and GST-ankZU5 were subcloned from GFP-ankG270 (rat) (a kind gift from Van Bennett) into pEGB vector. GST-unc5H1ZU5, GST-PIDDZU5 and GST-ZO1ZU5 were subcloned from myc-unc5H1 (a kind gift from Lindsay Hinck), Flag-PIDD (a kind gift from Jurg Tschopp) and ZO1 construct (a kind gift from W. Hunziker) respectively. The pcDNA3.1-HA-NRAGE (mouse) was a kind gift from Lindsay Hinck. The retroviral constructs were engineered either in pMSCV-IRES-Puro or pMSCV-IRES-GFP. The HA epitope tag was introduced at nterminal and NES/NLS at c-terminal of human NRAGE cDNA.

Anoikis assays: For caspase activity assays performed on confluent cells, 150,000 cells were plated per $35 \mathrm{~mm}$ well, and, after overnight attachment, transfected with $0.3 \mathrm{ml}$ of a $1.0 \mathrm{ml}$ mixture containing $15 \mu \mathrm{L}$ of $20 \mu \mathrm{M}$ siRNA and $15 \mu \mathrm{L}$ of lipofectamine RNAimax in a total of $2.3 \mathrm{ml}$ Opti-MEM medium (Invitrogen, Carlsbad, CA). Cells were refed with their normal growth medium 4-6 hours later and grown for 45 hours post-transfection, trypsinized, and resuspended in $1.5 \mathrm{ml}$ of medium per time point. Each time point was plated in one $35 \mathrm{~mm}$ Ultra-low attachment well (Corning), cells were collected by centrifuging for 15 seconds at 8,000 rpm in a microfuge tube, washed in ice-cold PBS and lysed in $0.2 \%$ Triton-X100/PBS+1mM DTT. After clearing lysates at 13,200 rpm for 10 minutes, samples were assayed for caspase activity in $100 \mu \mathrm{L}$ of caspase assay buffer (20 mM Pipes/100 mM NaCl/1 mM EDTA/0.1 \% Chaps/10 \% sucrose, $\mathrm{pH}$ 7.2/10mM DTT) containing $0.15 \mathrm{mM}$ Ac-DEVD-AFC substrate and read in a Molecular Devices Gemini XPS fluorimeter.

For subconfluent cells assayed by DNA fragmentation ELISA: $5 \times 10^{4}$ cells (MCF10a/HMLE) were plated in wells of 6 -well dishes. Two duplicate wells with target 
siRNA and two duplicate wells with control siRNA, using 500 uL of Opti-MEM containing 5 $\mu \mathrm{L}$ of $20 \mu \mathrm{M}$ siRNA and $5 \mu \mathrm{L}$ of lipofectamine RNAi-max, added to a well containing $2.0 \mathrm{ml}$ of Opti-MEM. SiRNAs for NRAGE and TBX2 were "SmartPools" (Dharmacon).

After 4-6 hours, cells were re-fed with regular growth medium, and, 24 hours later, each well was split into one $60 \mathrm{~mm}$ dish. These were further incubated overnight, trypsinized, and resuspended in $1.5 \mathrm{ml}$ of growth medium per time point that was plated in a $35 \mathrm{~mm}$ Ultralow Attachment well. At each time point, cells were spun down at $8,000 \mathrm{rpm}$ for 15 seconds in a microfuge tube, washed in ice-cold D-PBS and lysed in $200 \mathrm{uL}$ of 0.2\% TritonX100/PBS/10mM EDTA or Roche Cell Death ELISA Lysis/Incubation buffer that was supplemented with $0.2 \%$ TritonX100. (Note: without supplementation, this buffer did not lyse aggregated MCF10a cells efficiently). Lysates were cleared at 13,200 rpm for 10 minutes and, in the case of the first lysis buffer listed above, assayed for protein concentration using a Bio-Rad protein assay. Normalized amounts of lysate (usually in the range of 5-15 uL) were then assayed in a total of $100 \mathrm{uL}$ of Roche Cell Death ELISA lysis/Incubation buffer in the Roche system and read in a Molecular Devices plate-reading spectrophotometer. TBX2 knockdown was verified on a western blot using Santa Cruz pAb (clone H137) and NRAGE knockdown was verified using BD Biosciences clone $48 \mathrm{mAb}$.

Apoptosis in transiently transfected cells: The effect of ankyrin over-expression on apoptosis was measured by over-expressing individual constructs in H1299, 293T and cos7 cells and processing the cell lysates for DNA fragmentation ELISA. Briefly, $0.5-1 \mathrm{X}$ 105 cells were plated in 6-well plates. Next day, the cells were transfected using lipofectamine LTX (H1299) (Invitrogen, Carlsbad, CA, USA) or fugene 6 (293T or Cos7) 
(Roche, Indianapolis, IN, USA) with expression constructs (with or without siRNA) using the manufacturer recommendations. The cells were lysed after 40-48 hours of transfection and lysates were assayed for DNA fragmentation ELISA. The expression of each construct was confirmed by immunoblot analysis.

Cell fractionation: The MDCK cells were grown in cell culture inserts (Fisher Scientific, Pittsburgh, PA, USA) and allowed to grow till confluence. Once the cells were of required confluence, they were used either attached or detached (trypsinized). The cells were washed with 1XHBSS (Hanks Balanced Salt Solution, Invitrogen, Carlsband, CA, USA), solution followed by washing with ice cold hypotonic buffer ( $5 \mathrm{mM}$ Tris, $\mathrm{pH} 7.5$, $1 \mathrm{mM}$ EGTA, $5 \mathrm{mM} \mathrm{MgCl} 2$ and $1 \mathrm{mM}$ DTT with complete protease inhibitor) for 3 minutes. The cells were washed again with 1XHBSS followed by 200ul CSK (cytoskeletal) buffer (pH 6.8 PIPES 10mM, 300mM sucrose, 3mM MgCl2, $\mathrm{NaCl} 50 \mathrm{mM}$ and $0.5 \%$ Triton X100 with complete protease inhibitor) treatment for 3 minutes. Supernatant was collected and dissolved into equal amount of 2XSDS loading buffer and pellet was dissolved in 400ul of 1XSDS loading buffer followed by boiling for 5 minutes. The equal volume was used for immunoblot analysis.

Immunofluorescence: For ankyrin (Anti-rabbit 1:200) / e-cadherin (Anti-mouse 1:50) localization in sub-confluent and confluent cells, methanol fixed MDCK cells were used. Briefly cells were plated and grown for the required density, fixed and processed for immuno-fluorescence (IF) using above mentioned primary and compatible secondary antibodies (anti-mouse Alexa 488 and anti-rabbit Alexa 594, Invitrogen, Carlsband, CA, USA) and images were taken on Zeiss fluorescence microscope. The effect of ankyrinFL/DelUPA on NRAGE localization experiment was performed in HT1080 cells. Briefly, 
cells were co-transfected with HA-NRAGE and ankyrin/control constructs followed by $4 \%$ PFA fixation after 36-40 hours. The fixed cells were processed for IF using anti-HA (mouse, 1:500) and anti-ankyrin antibodies.

The effect of ankyrin depletion on NRAGE localization was performed in MCF10a cells. Briefly, $30-40 \%$ confluent cells were transfected using lipofectamine RNAimax (Invitrogen, Carlsband, CA, USA) as mentioned earlier. The cells were treated with 6nM leptomycin B for 30 minutes followed by $4 \%$ PFA fixation (15 minutes), PBS washing (3 times), permeabilization with $0.2 \%$ triton $\mathrm{X}-100$ for 5 minutes, washing with $100 \mathrm{mM}$ Glycine containing PBS-tween20 $(0.1 \%)$ for $3 \times 5$ minutes, primary antibody incubation for 1 hour, PBS-tween20 washing for $3 \times 5$ minutes, secondary antibody treatment for 30 minutes, PBS-tween20 washing for $3 \times 5$ minutes followed by fixing cells with DAPI.

The localization of NRAGE in HMLE, HMLE/shECAD and HMLE/ER-Twist cells were performed as follows. $1 \times 10^{5}$ cells were plated on cover slips and they were allowed to grow for 2-3 days. HMLE/ER-Twist cells were maintained in 4-OH-tamoxifen (after 10 days initial treatment). The cells were treated with 6uM leptomycinB followed by PFA fixation and IF as mentioned earlier. The nuclear and total NRAGE signals for individual images were quantitated using image j software $(\mathrm{NIH})$ and the average signal was compared for stastical significance using free online version of graphPad software (http://www.graphpad.com) and Microsoft excel software.

For the HT1080 co-transfection experiment, HA-mouse-NRAGE (Sasaki et al., 2002) in pcDNA3.1 was cotransfected (Fugene-HD) with 3XFLAG-ankyrin-G (210 kDa isoform) constructs generated in the vector p3XFLAG-CMV-10 (Sigma). Twenty-four 
hours later, coverslips were fixed and doubly stained with rat anti-HA mAb (Covance) and mouse anti-FLAG M2 (Sigma) using procedures outlined above.

Protein interaction methods: For co-immunoprecipitation of NRAGE with TBX2, MCF10a and HMLE cells (two confluent $100 \mathrm{~mm}$ dishes) were washed and lysed in standard IP buffer (25mM Tris pH8, 200mM NaCl, 10\% glycerol, 0.5\% Triton-X100, 1X complete protease inhibitor cocktail, Pierce). Lysates were pre-cleared at 13,200 rpm for 10 minutes and then pre-absorbed for 30 minutes with $65 \mu \mathrm{L}$ of $50 \%$ protein A-sepharose (GE Healthcare) that had been pre-equilibrated in standard IP buffer with $10 \mathrm{mg} / \mathrm{ml}$ BSA $+10 \mathrm{mg} / \mathrm{ml}$ ovalbumin. After clearing, $3 \mu \mathrm{g}$ of NRAGE pAb (Santa Cruz sc-28243) or normal IgG (Covance) was added and samples were incubated 2 hours at 4 degrees with rotation. Protein A-sepharose $(40 \mathrm{uL})$ was then added and incubation was continued for an additional hour. After three washes with standard IP buffer, samples were boiled in $2 X$ SDS sample buffer (containing 10\% $\beta$-mercaptoethanol), loaded onto a pre-cast $4-20 \%$ polyacrylamide gel (Invitrogen, Carlsbad, CA) and analyzed on a western blot for TBX2 (Santa Cruz pAb sc-48780) or NRAGE (BD Biosciences mAb, clone 48); Clean-blot secondary antibody (Pierce) and anti-mouse-HRP (BioRad) were used respectively for the detection of these antibodies, followed by development with West Pico ECL reagent (Pierce).

For detection of ankyrin-NRAGE complexes, the method above was modified as follows. Five minutes prior to lysis, cells were washed and incubated on ice with hypotonic buffer (5mM Tris pH 7.5/1mM EGTA/5mM MgCl2/1mM DTT/1X complete protease inhibitors, Pierce). This was found to result in better ankyrin-G detection, presumably due to the influx of protease inhibitors into the cells prior to lysis. Following 
immunoprecipitation as above, proteins were transferred to PVDF membranes overnight at $30 \mathrm{~V}$ in transfer buffer with the methanol reduced to $5 \%$. Ankyrin-G was detected using a Santa Cruz pAb (sc-28561), a pAb kindly provided by Vann Bennett (Duke University) or pAB kindly provided by Deborah Anderson (Saskatchewan Cancer Institute, Saskatoon, Canada). For ankyrin-G immunoprecipitation, antibody was developed against the C-terminal domain.

For transient co-transfections, 293T cells were plated in $35 \mathrm{~mm}$ collagen-coated wells and co-transfected, using $150 \mu \mathrm{L}$ per well of cells (in $2.0 \mathrm{ml}$ of Opti-MEM) of a solution containing $200 \mu \mathrm{L}$ Opti-MEM, $1 \mu \mathrm{g}$ of each expression construct ( $2 \mu \mathrm{g}$ total DNA) and $6 \mu \mathrm{L}$ of Fugene-HD (Roche). After four hours, cells were returned to normal growth medium (DMEM+10\% fetal bovine serum+1X penicillin-streptomycin-glutamine). Twentyeight to 36 hours later, cells were collected by pipetting each well in $1.0 \mathrm{ml}$ of cold PBS, pelleted in a microfuge at 8,000 rpm for 15 seconds, and lysed in standard IP buffer (600 $\mu \mathrm{L}$ ), followed by preclearing the lysates at $13,200 \mathrm{rpm}$ for 10 minutes. For GST pulldowns or FLAG pulldowns, $40 \mu \mathrm{L}$ of glutathione-sepharose (GE Healthcare) or FLAGagarose (Sigma), pre-equilibrated in standard IP buffer $+10 \mathrm{mg} / \mathrm{ml}$ BSA $+10 \mathrm{mg} / \mathrm{ml}$ ovalbumin, was added, and samples were rotated at 4 degrees for $1.5-2$ hours. Following three washes in standard IP buffer, GST pulldowns were eluted with $40 \mu \mathrm{L}$ of 2 X SDS sample buffer (containing 10\% $\beta$-mercaptoethanol), by boiling. FLAG pulldowns were eluted with $50 \mu \mathrm{L}$ of $0.1 \mathrm{M}$ glycine $\mathrm{pH} 2.8$ at 4 degrees for 10 minutes; after removal of the beads by spinning, $40 \mu \mathrm{L}$ of the supernatant was neutralized by the addition of $8.8 \mu \mathrm{L}$ of 0.5M Tris $\mathrm{pH} 7.5$. An equal volume of $2 \mathrm{X}$ SDS sample buffer (containing $10 \% \beta$ mercaptoethanol) was added and all samples were analyzed on western blots for GST 
(anti-GST mAb, Santa Cruz), FLAG (anti-FLAG M2, Sigma) or HA (HA.11 mAb Ascites, Covance).

Expression constructs used for the interaction domain mapping experiments were as follows: To generate GST-NRAGE fusion expression constructs, NRAGE sequences were amplified (Phusion polymerase, NEB) from the Origene clone, subcloned into the Xhol-Hindlll site of pcDNA3.1mychis-A(-) and then re-subcloned into the Sall-Notl site of the mammalian GST fusion expression vector pEBG2. The full-length mouse TBX23XFLAG expression construct was kindly provided by Colin Goding (Oxford University). For the experiments mapping the domain of NRAGE that interacts with ankyrin, the GSTNRAGE fusion constructs were co-transfected with a clone, kindly provided by Deborah Anderson (Saskatchewan Cancer Institute, Saskatoon, Canada) that expresses the p105 isoform of mouse ankyrin-G in the vector p3XHA. For experiments to map the domain of ankyrin-G that interacts with NRAGE, human ankyrin-G fragments from the Imagenes (Berlin, Germany) clone DKFZp686P17114, containing accession number BX537917, were amplified by PCR as BamHI-Hpal fragments and subcloned into the Bglll-Hpal site of the p3XHA vector.

Soft agar assays: SW13 adrenocortical carcinoma cells (ATCC) were maintained in DME $+10 \%$ fetal bovine serum +1 Xpenicillin-streptomycin-glutamine. SW13 cells overexpressing TBX2 were generated by infection with mouse TBX2-FLAG that had been subcloned into the retroviral vector pMIG followed by flow-sorting for GFP positive cells and western blotting to confirm expression. Stable NRAGE knockdown derivatives were then generated by infection of these TBX2-expressing cells with an NRAGE-shRNA retrovirus in the vector pRS (Origene). The MDA-MBA-435 cells were also generated 
using NRAGE-shRNA retrovirus. $1.0 \mathrm{ml}$ of $0.7 \%$ low melting agarose in complete medium was mixed with 10,000 SW13-TBX2/pRS or SW13-TBX2/shNRAGE cells and plated onto 6 wells each of 24-well ultra low attachment plates (Costar).

The cells were grown for 7-10 days and soft agar media was refed each 4-5 days. 500 cells of SW13-TBX2/pRS and SW13-TBX2/shNRAGE cells were plated onto three wells of tissue culture treated 6-well plates and grown for 7-10 days. Six images from each well of soft agar colonies ( $>8$ cells/colony) were photographed using 10X magnification lens (Zeiss Axiovert 200M) and averaged. The averaged soft agar colonies were normalized against the attached colony numbers. 


\section{CHAPTER 3}

\section{ROLES OF ANKYRIN AND INTERACTING PROTEINS IN ANOIKIS}

Introduction

Ankyrin is a family of cortical cytoskeletal proteins, known to link membrane proteins to the actin cytoskeleton. The epithelial isoform of ankyrin, ankyrin-G required in membrane biogenesis, targeting of various membrane proteins including the adherens junction protein E-cadherin and $\mathrm{Na}^{+}-\mathrm{K}^{+}-\mathrm{ATPase}$, etc. (Kizhatil and Bennett, 2004; Kizhatil et al., 2007). Ankyrin-G is known to have pro-apoptotic domains such as ZU5 and death domain, implicated in regulation of apoptosis (Feinstein et al., 1995; Williams et al., 2003). The sub-membrane localization and presence of pro-apoptotic domains (ZU5 and death domains) indicated that ankyrin-G might be important for regulation during cellextracellular matrix dissociation induced apoptosis (anoikis) (Frisch and Francis, 1994).

The role of ankyrin-G in apoptosis hasn't been characterized well. The evidence from renal ischemia reperfusion studies provided some experimental evidence. The MDCK cells depleted for ATP abolished ankyrin-spectrin association, mislocalization of ankyrin$\mathrm{Na}^{+}-\mathrm{K}^{+}-\mathrm{ATPase}$, and the loss of $\mathrm{Na}^{+}-\mathrm{K}^{+}-\mathrm{ATPase}$ function affecting ion homeostasis (Woroniecki et al., 2003). In cells undergoing ischemia reperfusion, ankyrin-G associated strongly with Fas and FADD and induced the apoptotic response (Del Rio et al., 2004). Ankyrin-G has been found to overexpress in the fibroblast of HGPS (Hutchinson-Gilford Progeria Syndrome) patients, a pre-mature aging disease and correlated with increased apoptosis (Wang et al., 2006). Ankyrin-G has also been shown to downregulate in multiple types of cancer (Glinsky et al., 2005). Few reports have indicated the role of 
ankyrin-G in promoting metastasis by interaction with CD44 and Tiam1 (Bourguignon, 2001). Our study reveals the biological role of ankyrin-G through its interaction partners. Results

Ankyrin-G colocalizes with E-cadherin: It is established that the epithelial adherens junction protein E-cadherin is a molecular partner of ankyrin-G (Kizhatil et al., 2007; Nelson et al., 1990). Ankyrin-G, which is required for lateral membrane biogenesis, helps E-cadherin to localize in lateral membranes (Kizhatil and Bennett, 2004; Kizhatil et al., 2007). The co-immunofluorescence microscopy experiment in MDCK (Madrin-Darby canine kidney) cells demonstrated the co-localization of E-cadherin and ankyrin-G in interacting (confluent) and non-interacting (subconfluent) cells (fig.1a). These two proteins exhibit diffused cytoplasmic localization in non-interacting cells, whereas they shifted to membrane junctions in interacting cells. The MDCK cells were also used for subcellular fractionation experiments and despite the cellular localization, ankyrin-G remained associated with the cytoskeletal fraction (fig.2b). Additionally, a recent publication reported that the loss of E-cadherin leads to anoikis resistance and increased metastasis (Onder et al., 2008). We used human mammary epithelial (HMLE) cells and the cells which have undergone epithelial-mesenchymal-transition such as HMLE-shECAD (stable knockdown of E-cadherin) and HMLE/ER-Twist (4-OH tamoxifen inducible Twist) cells. The expression of ankyrin-G correlated with E-cadherin and downregulated during EMT (fig.1c). These data suggest that E-cadherin is required for the expression/stability of ankyrin-G in an epithelial cell. 


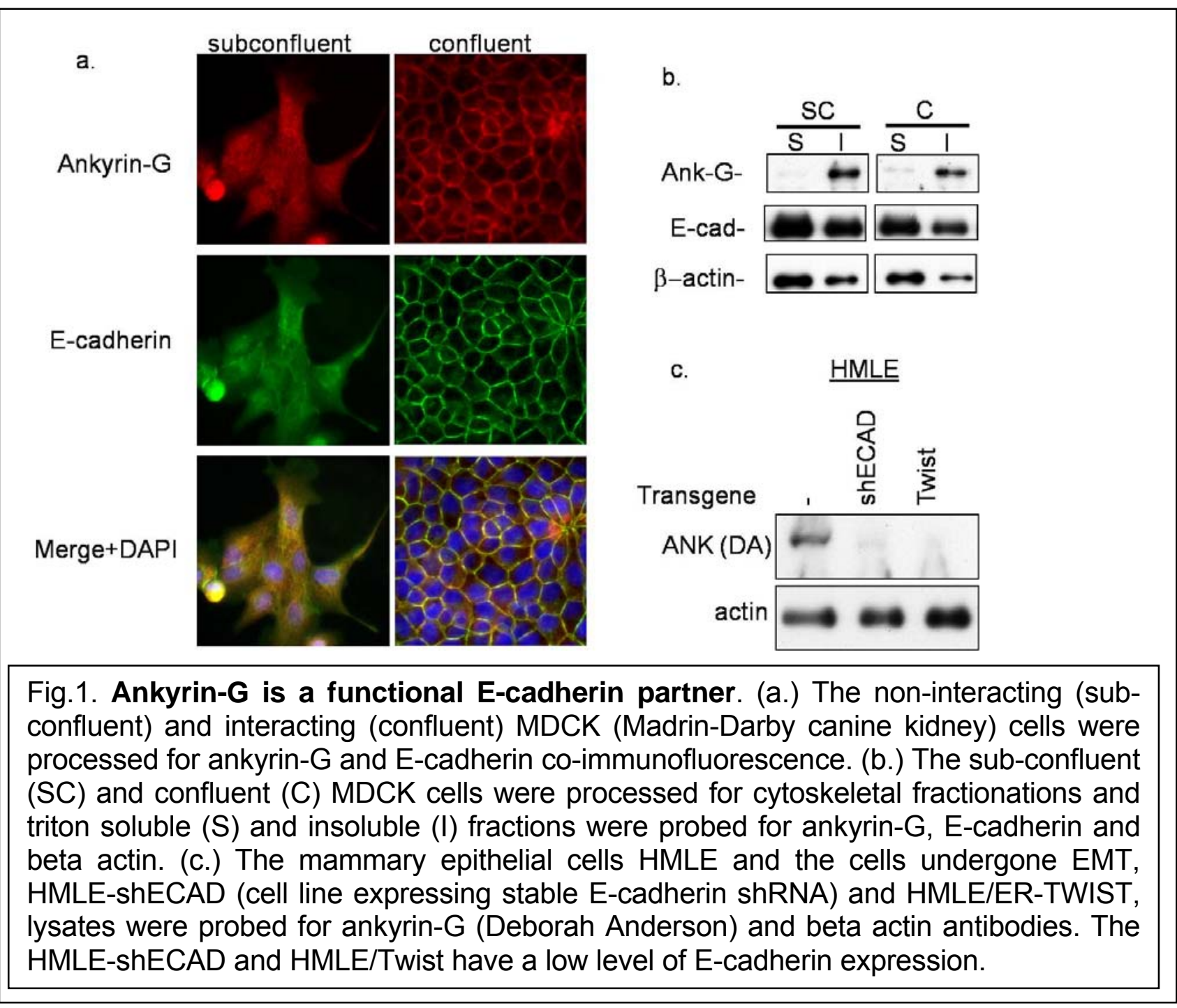

The effect of cell-extracellular matrix dissociation was tested on ankyrin-G in MDCK cells (fig.2). In contrast to cytoskeletal localization in interacting cells, ankyrin-G relocalized to the cytoplasmic pool in the early detached fractionated cell lysates. In these lysates, GAPDH remained cytosolic whereas beta actin pre-dominantly remained cytoskeletal. Spectrin beta2, the cytoskeletal partner of ankyrin-G also re-localized significantly to cytoplasm. We hypothesized that the re-localization of ankyrin-G from cytoskeleton to cytoplasm must be critical for regulation of anoikis in epithelial cells. 


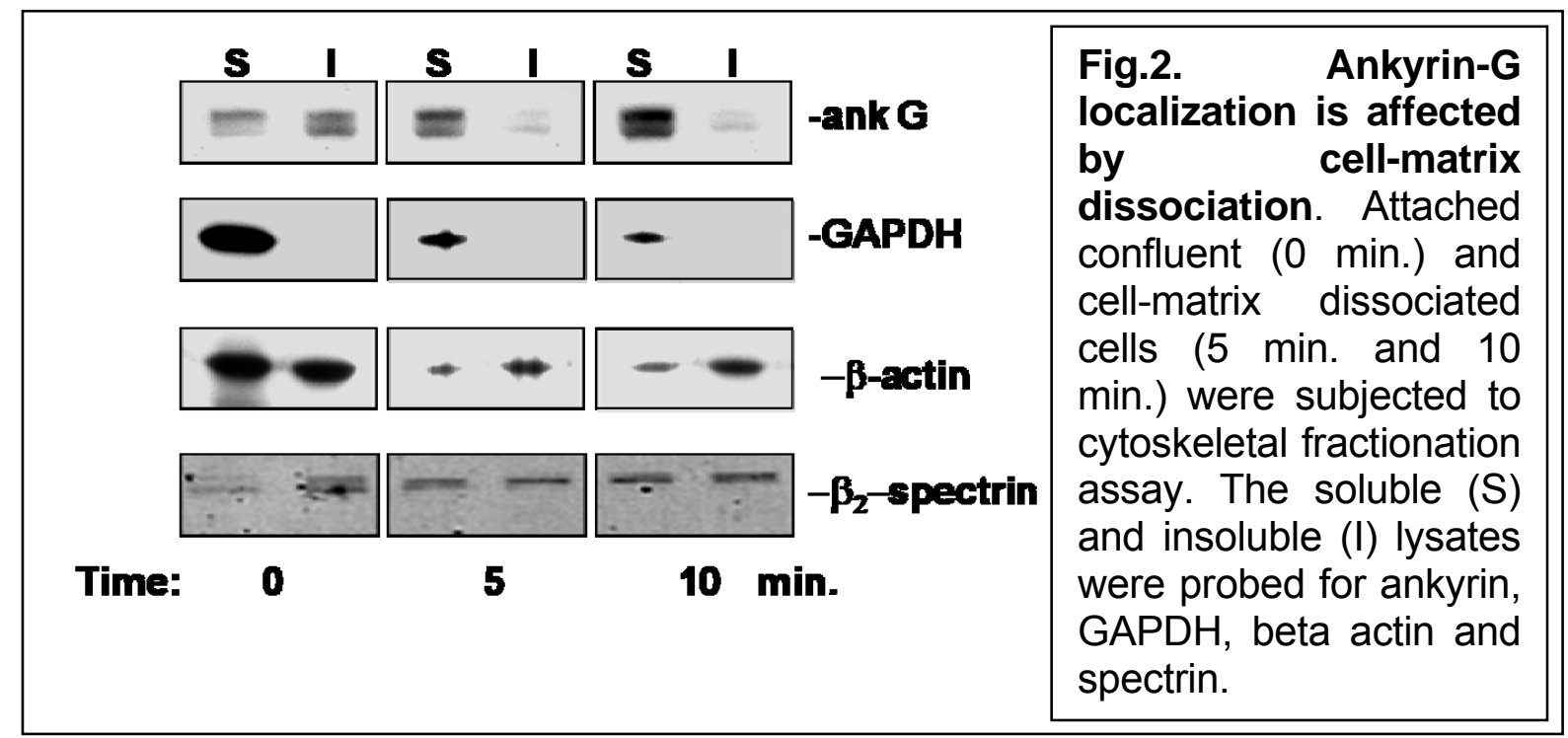

Effect of suspension on ankyrinG-spectrinßII interaction: Loss of cell-extracellular matrix interaction resulted in localization of ankyrin-G from cytoskeleton to cytoplasm. We further tested the effect on cell-extracellular dissociation on ankyrin-G and spectrin-beta2 interaction in MCF10a cells. The lysates from attached and suspended cells were immunoprecipitated with ankyrin-G and blotted either with ankyrin-G or spectrin-beta2. The data suggested that the interaction between ankyrin-G and spectrin-beta2 was lost during early suspension (30 minutes suspension). The enrichment of predominantly cytoplasmic ankyrin-G may result in loss of ankyrin-spectrin interaction. We also tested the effect of suspension on ankyrin-G protein level. The cells were suspended upto 24 hours and lysates were subjected to ankyrin-G immunoblot. The data suggested that ankyrin-G protein level increased during suspension. It is not clear if the increase was due to transcription/translation or result of protein stability. Loss of cell-extracellular matrix interaction promotes apoptosis (anoikis), and an increase in ankyrin-G level correlates as a positive regulator of anoikis. 


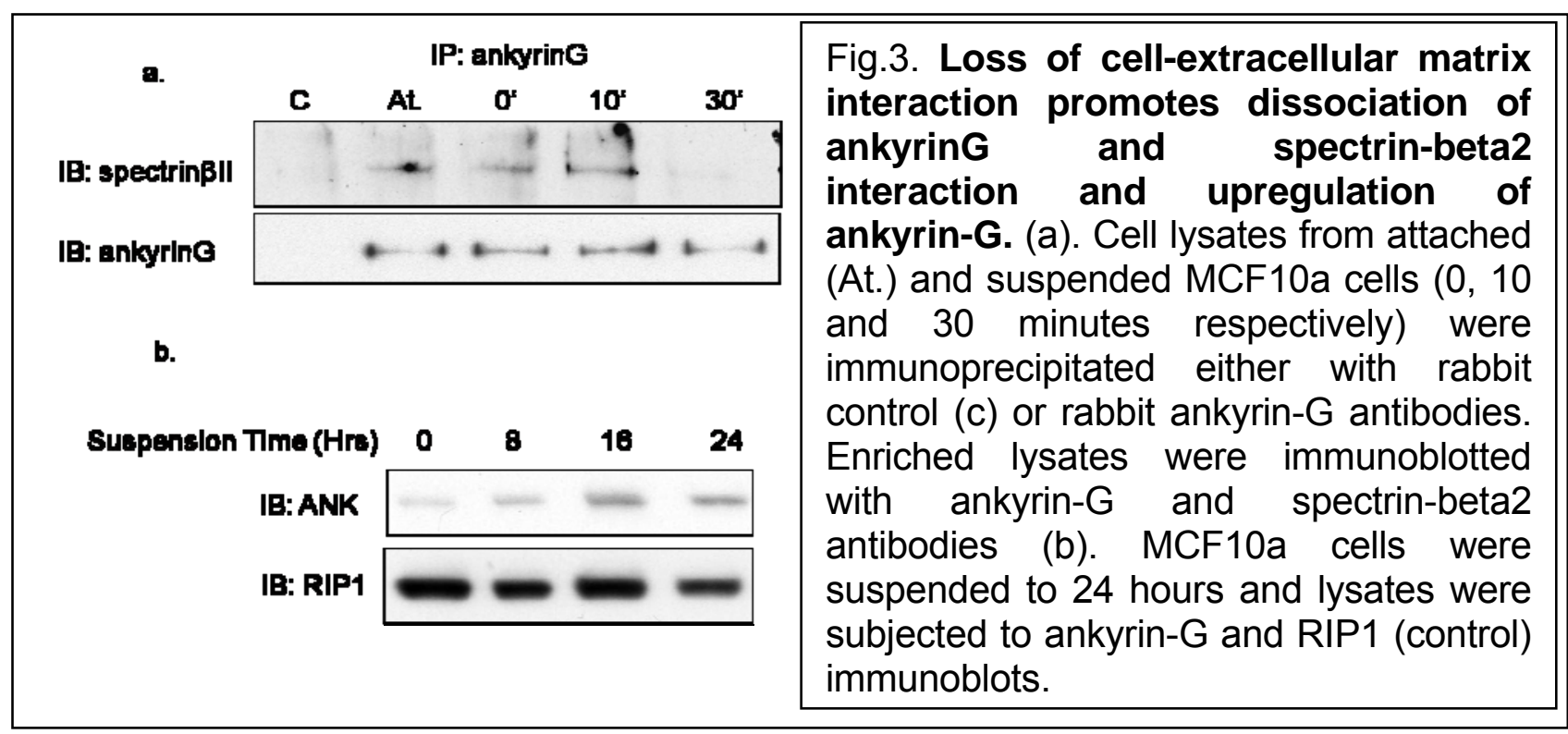

Overexpression of ankyrinG induced apoptosis through ZU5 and death domain:

The cell-extracellular matrix dissociation results in re-localization of ankyrin-G from cytoskeleton to cytoplasm (fig.2). The relocalization resulted in ankyrinG-spectrin beta2 dissociation and an increased ankyrin-G level (fig.3). The cytoplasmic ankyrin-G and its upregulation could be critical in anoikis initiation. To test the effect of ankyrin-G overexpression, we used the transient overexpression strategy in various mammalian cell lines. The overexpression of ankyrin resulted in induction of apoptosis in several cell lines such as 293T, cos7, and H1299. Interestingly, the overexpression of ankyrin-G correlated with increased apoptosis in fibroblasts in HGPS patients (Wang et al., 2006). We further tested the effect of individual domains by either using deletion constructs (delZU5 or delDD or delZU5-DD) or domain specific constructs (ZU5 or DD or MBD). Based on the apoptosis data (DNA fragmentation assay), overexpression of ankyrin-G induced apoptosis was dependent on ZU5 and death domain and the construct lacking ZU5/death domain with only ankyrin repeats (MBD) failed to induce apoptosis (fig.4). In principle, this apoptosis could occur through multiple mechanisms, either through the individual ZU5 
domain or the individual death domain. Consistent with this duality of mechanisms, the deletion of the ZU5 domain or the death domain alone had only a minor effect on apoptosis. The ankyrin repeat/membrane binding domain (MBD), which mediates ankyrin interactions with membrane proteins, did not induce apoptosis, indicating that the deletion of both the ZU-5 and death domains abrogated apoptosis. This result also indicated that the severance of actin cytoskeleton-membrane association was insufficient for apoptosis induction. The ZU5 domian only and delZU5 constructs induced apoptosis equally well whereas ankyrin death domain (ankDD) only and deletion domain (delDD) construct of ankyrin significantly induced apoptosis, suggesting that the apoptotic mechanisms are different. The death domain is the major domain in apoptosis induction whereas ZU5 domain mediated apoptosis might be result of interference with spectrin-ankyrinG interaction.

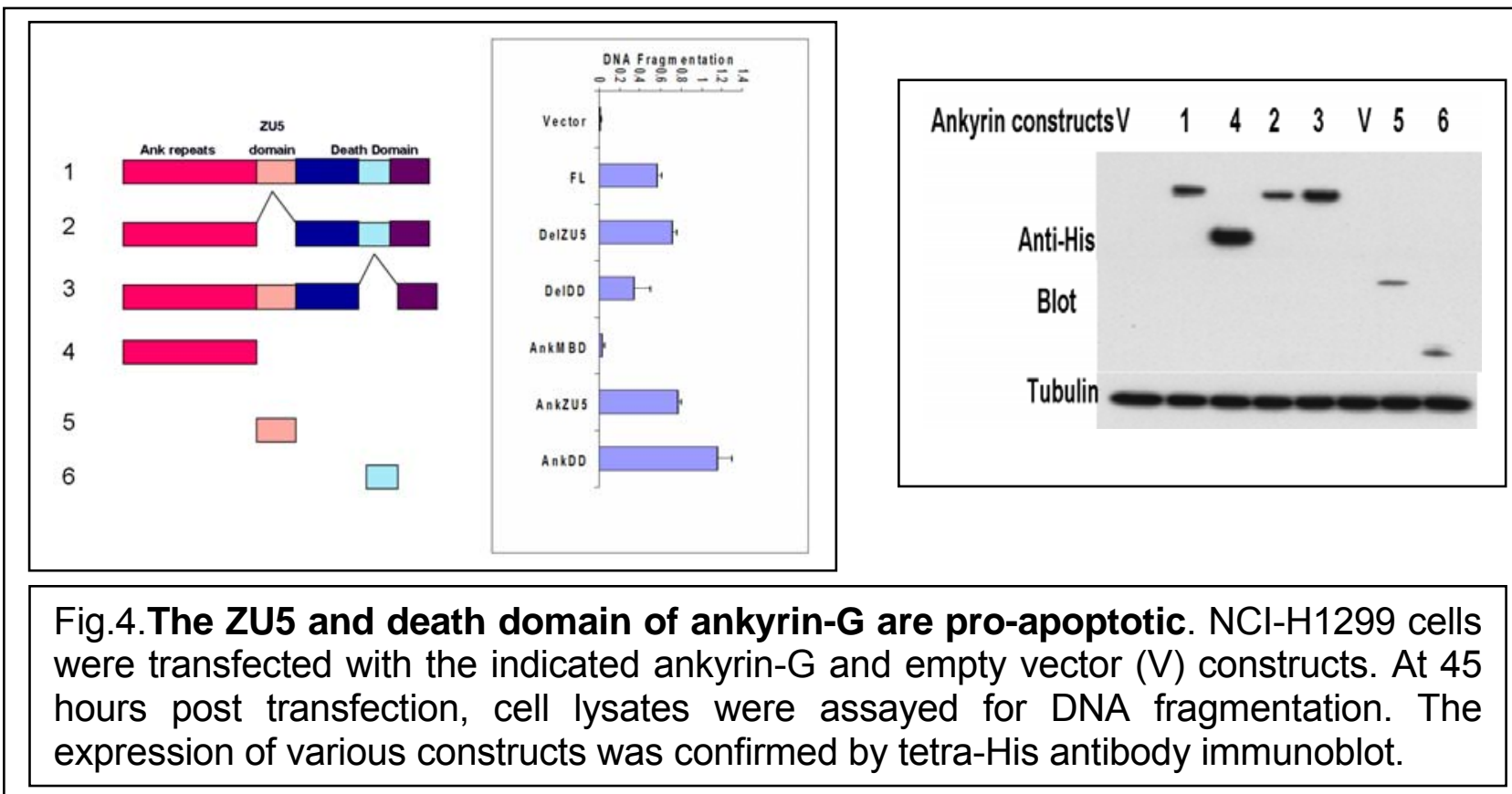


Transient knockdown of ankyrin-G enhances anoikis: Epithelial-mesenchymaltransition (EMT) results in downregulation of ankyrin-G (fig. 1c). EMT correlates with anoikis resistance. Transient overexpression of ankyring induced apoptosis in various epithelial and cancer cells (fig. 3). Based on the preliminary data, we hypothesized that ankyrin-G to be an anoikis promoter. We tested the effect of ankyrin-G knockdown on anoikis in mammary epithelial cells (MCF10a). Contrary to our hypothesis, knockdown of ankyrin-G increased the anoikis response, suggesting its role as a possible pro-survival protein.

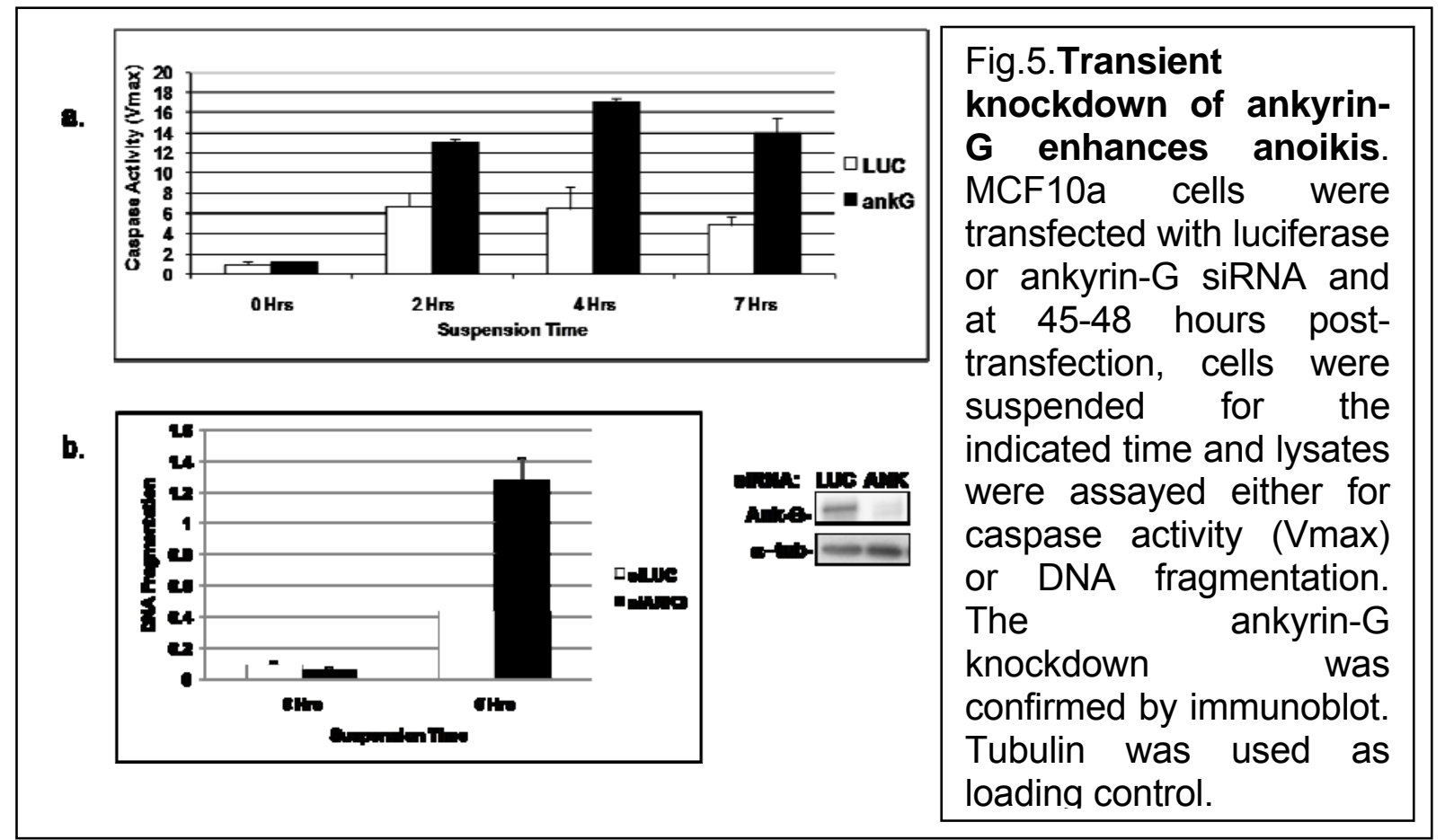

Ankyrin-G interacts with various other membrane and cytoplasmic proteins involved in adhesion, cytoskeletal assembly and apoptotic processes. It can affect apoptosis/anoikis by multiple protein interactions and cellular functions. Ankyrin is downregulated by various tumor promoting agents in many tumor types. The effect of ankyrin-G depletion on anoikis is unexpected but it can be explained by various 
interactions such as CD44 and $\mathrm{Na}^{+}-\mathrm{K}^{+}$-ATPase and related pro-survival function. It is reported that inhibition of $\mathrm{Na}^{+}-\mathrm{K}^{+}$-ATPase by its inhibitor ouabain results in enhanced anoikis in cancer cells and prevents distant tumor formation (Simpson et al., 2009). The absence of ankyrin-G from the cytoskeleton has been reported to affect $\mathrm{Na}^{+}-\mathrm{K}^{+}$-ATPase localization and activity (Woroniecki et al., 2003) during ischemia reperfusion and ATP depletion. It is also conceivable that certain splice variants and/or post-translational modifications of ankyrin-G may have alternate cellular functions.

Effect of dominant negative form of ankyrin (ANK-105) overexpression on anoikis:

\begin{tabular}{|l|l|l|}
\hline & \\
\hline
\end{tabular}

Functional ankyrin-G contains the membrane binding domain (MBD), which contains 24 ankyrin repeats (AR), spectrin binding domain (ZU5-A), ZU5-UPA-DD supermodule and the C-terminal domain. There are splice variants of ankyrin-G which do not contain the membrane binding domain (MBD) and they have been named according to their sizes i.e. ANK-105 and ANK-120, respectively (Ignatiuk et al., 2006). These variants can interact with spectrin but can't interact with membrane proteins such as E-cadherin, CD44, and $\mathrm{Na}^{+}-\mathrm{K}^{+}$-ATPase. We tested the effect of overexpression of the dominant negative form of ankyrin-G on anoikis in MCF10a cells. Over-expression of ANK-105 resulted in anoikis suppression in MCF10a cells. Considering that this splice variant doesn't link membrane 
proteins to the actin cytoskeleton and acts as a dominant negative, a functional cytoskeletal ankyrin-G might act as positive regulator of anoikis.

Loss of ankyrin-G destabilizes EGFR: Transient knockdown of ankyrin-G enhanced anoikis. This was contrary to the observation that ankyrin-G is downregulated during EMT. Anoikis and EMT data in turn suggest that ankyrin-G may have a proapoptotic as well as pro-survival function depending upon the localization, interaction partners and cellular contexts. We hypothesized that ankyrin-G might be supporting prosurvival function of membrane proteins and so its loss would enhance anoikis. To understand this phenomenon, we knocked down ankyrin-G and looked at signaling proteins in attached and cell-extracellular matrix dissociated MCF10a cells. The loss of ankyrin-G resulted in destabilization of EGFR at 6 hours of cell suspension (Fig. 7a). In another experiment performed in HMLE cells, knockdown of E-cadherin and ankyrin resulted into destabilization of full length EGFR $(\sim 180 \mathrm{kDa})$ and increased the $\sim 42 \mathrm{kDa}$ cleavage product (Fig. 7b). Expression of EGFR is associated with cell survival and proliferation and is known to be overactive in many carcinoma types. EGFR is shown to activate various pro-survival activities such as activating MAP Kinase pathways, suppression of pro-apoptotic proteins such as Bim and stabilization of the sodium/glucose cotransporter 1 (SGLT1) (Collins et al., 2005; Reginato et al., 2003; Weihua et al., 2008). Ankyrin-G mediated stabilization of EGFR is a novel finding, suggesting its possible role in EGFR membrane localization and stability. 


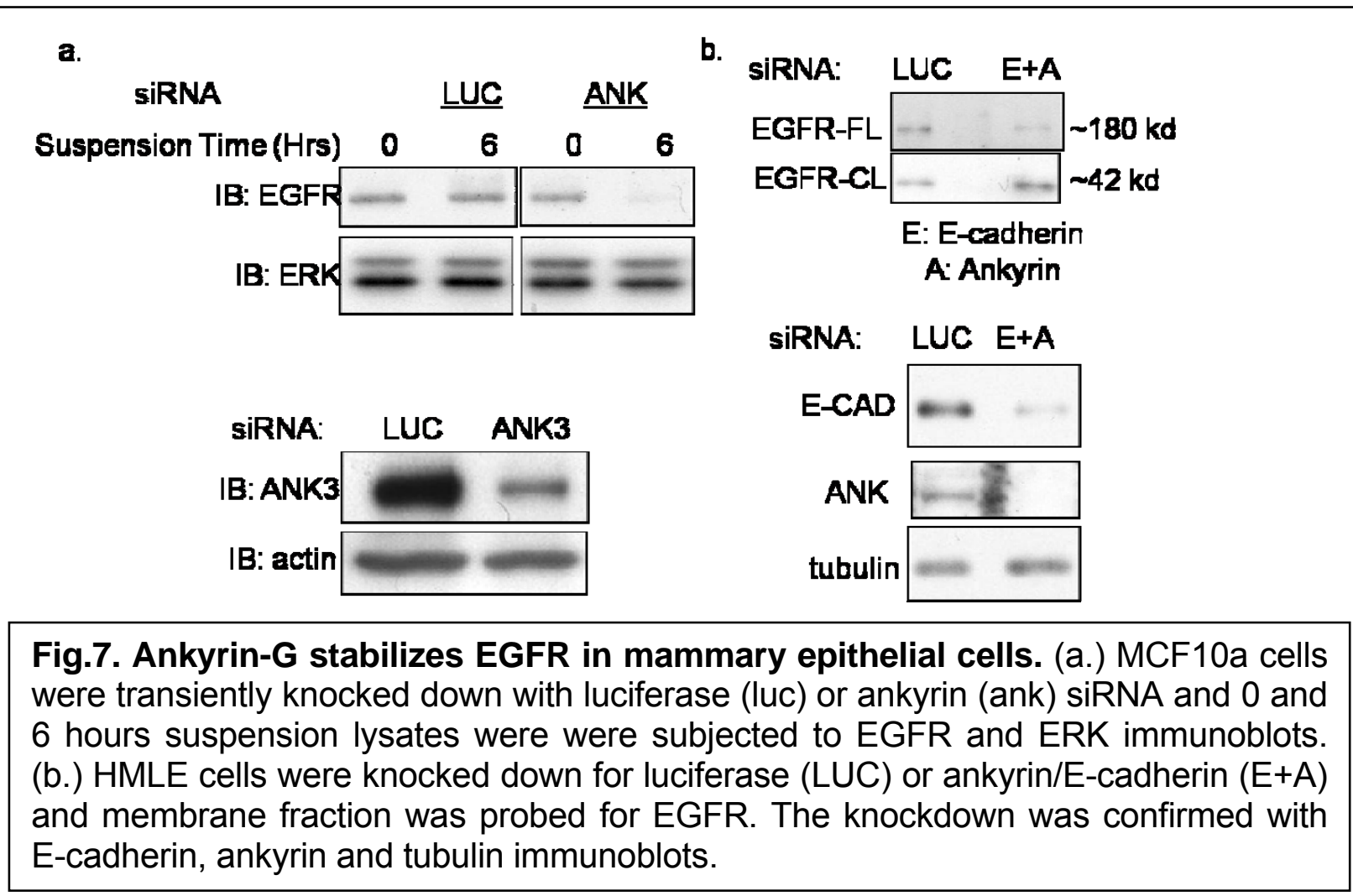

Ankyrin-G interacts with death domain proteins RIP1 and TRADD: Ankyrin-G contains a death domain, known for forming homo- and hetero-dimers. To monitor the ankyrin death domain interaction partners, we screened other death domain proteins for interaction. The interaction screen was performed through cotransfection of GST-ankDD and other death domain proteins in 293T cells, and lysates were enriched with glutathione sepharose beads. The ankyrin death domain (ankDD) interacting partners were members of the TNF signaling pathway (i) RIP1 (Receptor interacting protein with death domain), and (ii) TRADD (TNF receptor interacting adaptor containing death domain). RIP1 interaction was further confirmed in MCF10a, HMLE and MDCK cells. RIP1 has previously been demonstrated for anoikis regulation (Kamarajan et al., ; Kurenova et al., 2004). 


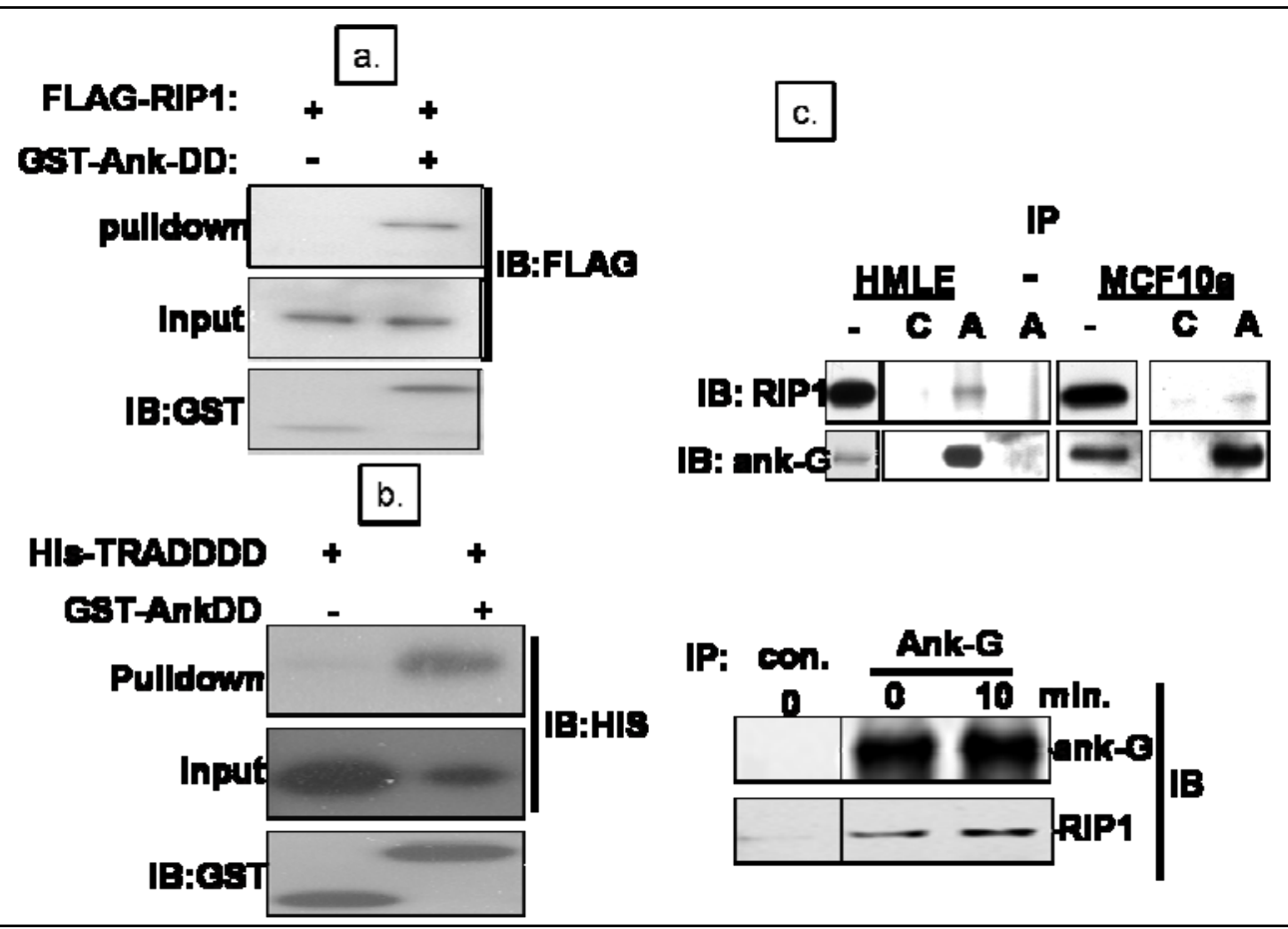

Fig.8. Ankyrin-G interacts with death domain proteins RIP1 and TRADD. (a.) FLAGRIP1 or (b.) His-tagged death domain of TRADD (His-TRADDDD) construct was cotransfected with GST or GST-ankyrinDD and lysates were enriched with glutathione sepharose beads. The pulldown samples were blotted for GST and FLAG (for RIP1) or His (TRADD-DD). (c.) The lysates of MCF10a and HMLE cells (upper panel) or attached (0 min.) and detached (10 min.) MDCK cells were immunoprecipitated with rabbit control lgG (C) or Ank-G (A) and samples were immunoblotted with ankyrin-G and RIP1.

Ankyrin induced apoptosis is RIP1 dependent: The ankyrin-G interacting proteins RIP1 and TRADD are well characterized for their roles in TNF mediated anti-apoptotic and proapoptotic pathways. RIP1 functions through a switch mechanism and its cleavage by caspase-8 promotes the pro-apoptotic function. We tested the role of RIP1 in ankyrin-G induced apoptosis and RIP1 was depleted by transient knockdown. Interestingly, ankyrinG induced apoptosis was partially rescued by the RIP1 depletion in $\mathrm{NCl}-\mathrm{H} 1299$ cells (Fig.8). 

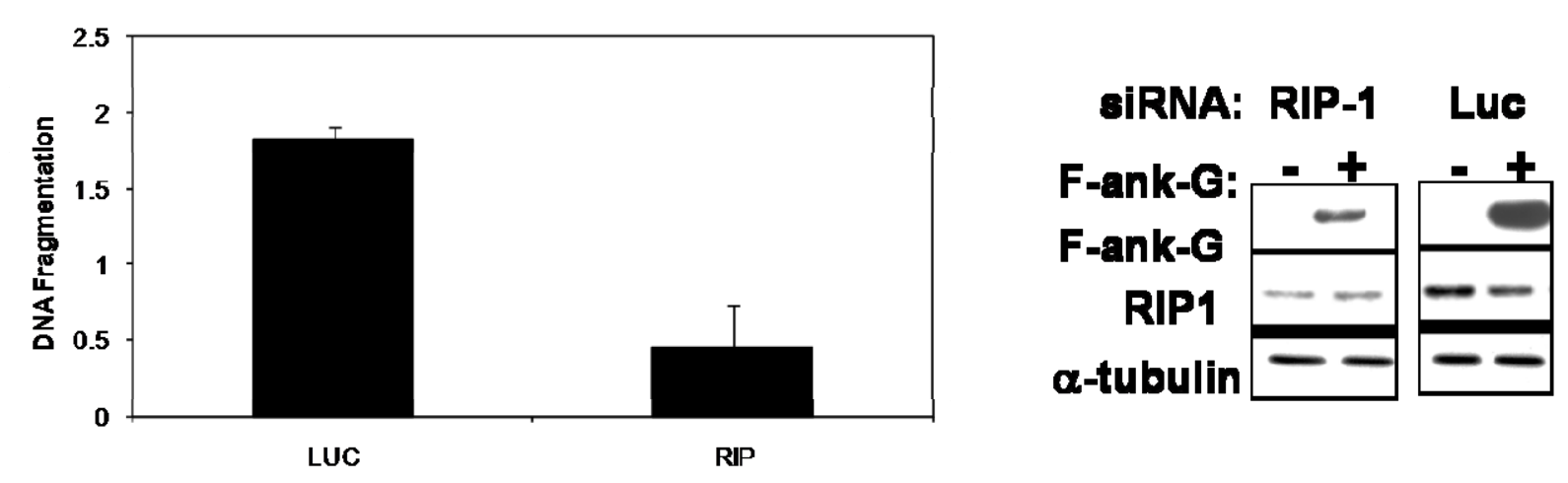

Fig.9. RIP1 promotes ankyrin-G dependent apoptosis. H1299 cells were cotransfected either with vector/ankyrin-G expression constructs and luciferase (Luc)/RIP1 siRNA and lysates were processed for DNA Fragmentation assay. Expression of ankyrin-G and depletion of RIP1 was confirmed by anti-His and RIP1 immunoblots. Alpha tubulin immunoblot was performed as loading control.

RIP1 is a known regulator of anoikis (Kurenova et al., 2004). RIP1 counteracts FAK pro-survival function by interacting with its $\mathrm{N}$-terminal domain. This observation has recently been extended by confirming this interaction and RIP1 shuttling between death domain receptor mediated pro-apoptotic pathway and FAK mediated pro-survival signaling (Kamarajan et al.).

Modulation in TRADD expression enhances anoikis: TRADD (TNFR1-associated death domain protein) is an adaptor protein known to link TNF receptor with Caspase-8 and RIP1 and modulate pathway by activating a pro-survival pathway activating NF-kB or pro-apoptotic pathway activating caspase-3. Our interaction screen identified TRADD as an ank-DD interaction partner. We tested the role of TRADD in anoikis either by retroviral mediated stable expression or transient knockdown in mammary epithelial cells MCF10a. Surprisingly, either over-expression or transient knockdown of TRADD enhanced anoikis compared to control cells. These surprising data suggest a novel function of TRADD in anoikis, which may possibly modulate TNF receptor pathway for NF-kB/caspase-3 
activation, which is yet to be characterized. It will also be interesting to understand how this function of TRADD is regulated by ankyrin-G.

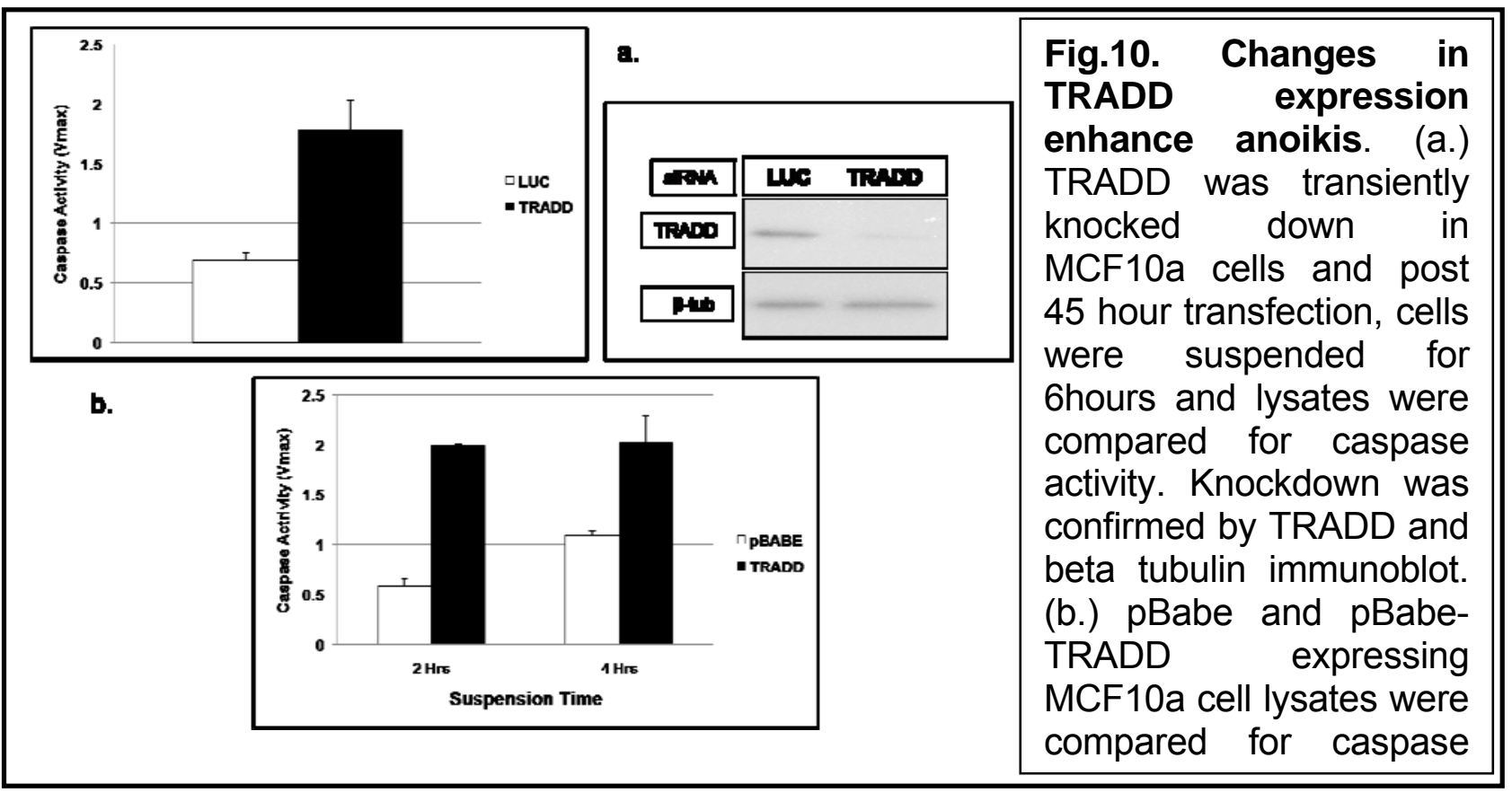

Discussion

Ankyrin-G is required for targeting interacting membrane proteins to the cytoskeketon (Ango et al., 2004; Mohler and Bennett, 2005). In our study, we reiterated the functional relationship between E-cadherin and ankyrin-G. Apart from co-localization in non-interacting and interacting cells, we found that ankyrin-G was downregulated in epithelial cells with E-cadherin stable knockdown (HMLE+shECAD), probably by activating an unknown transcriptional pathway (Onder et al., 2008). The cell fractionation experiments showed the cytoskeletal association of ankyrin-G in non-interacting and interacting cells. Surprisingly, ankyrin-G dissociated from the cytoskeleton and moved to detergent soluble cytoplasmic fraction in early detached cells, with loss of spectrin interaction and increase in ankyrin-G protein level. Incidentally, this observation correlates 
with events during ATP depletion/renal ischemia reperfusion which also resulted in loss of ankyrin-spectrin association in a time dependent manner (Woroniecki et al., 2003). The cells lacking extracellular matrix association are known to have metabolic defects (low ATP level) (Schafer et al., 2009). The data suggest that change in ankyrin-G localization in early detached cells may be involved in initiating anoikis as the cells lacking ankyrin (HMLE+shECAD) are resistant to anoikis. The overexpression of ankyrin-G in Cos7 and H1299 (express very low endogenous ankyrin-G, unpublished observation) cells, induced apoptosis. The apoptosis was dependent on presence of ZU5 and the death domain. These data correlate with Fas ligand induced apoptosis in ankyrin-G expressing MDCK cells which required death domain (Del Rio et al., 2004). The ZU5 effect on apoptosis correlates with a previous report suggesting that the expression of spectrin binding domain of ankyrin-R made HeLa cells detach from culture within few hours of transfection, probably as a dominant negative function (Kolondra et al., 2008).

We tested the effect of ankyrin-G knockdown on anoikis in MCF10a cells. Surprisingly, the knockdown of ankyrin-G enhanced anoikis sensitivity, suggesting its prosurvival function. Ankyrin-G pro-survival function can be explained by its interaction with $\mathrm{Na}^{+}-\mathrm{K}^{+}-\mathrm{ATPase}$ and $\mathrm{CD} 44$ interactions. Cell treatment with $\mathrm{Na}^{+}-\mathrm{K}^{+}$-ATPase inhibitor ouabain enhances anoikis response in cancer cells (Simpson et al., 2009). Ankyrin-G is required for $\mathrm{Na}^{+}-\mathrm{K}^{+}-\mathrm{ATPase}$ localization and function. In absence of ankyrin-G, $\mathrm{Na}^{+}-\mathrm{K}^{+}-$ ATPase failed to localize to the lateral membrane and the mislocalized enzyme loses its activity (Kizhatil and Bennett, 2004; Woroniecki et al., 2003). Ankyrin-G promotes CD44 mediated ovarian, breast and prostate carcinoma (Bourguignon, 2001; Bourguignon et al., 2008). The knockdown of CD44 enhances anoikis sensitivity in MCF10a cells (Ben 
Cieply, unpublished data). The ankyrin-G knockdown in CD44 independent cells such as HMLE may exhibit a different response. Preliminary data suggest that ankyrin-G knockdown doesn't affect anoikis in HMLE cells which needs to be verified. There are possibilities that different splice variants, post-translational modification and proteolytic cleavage products affect ankyrin-G function. We have observed that the ankyrin-G undergoes proteolytic cleavage in MCF10a cells, whereas cells dissociated from extracellular matrix lose it. In tumor tissue lysates, we have observed various large forms of ankyrin-G compared to mostly proteolytic cleaved forms of ankyrin-G in normal tissue lysates (personal observation). The phospho-tyrosine enriched ankyrin- $G$ was identified in the immunoaffinity profiling of phosphorylated tyrosine in cancer cell lines, suggesting its possible post-translational regulation during tumor progression (Rush et al., 2005). Our data also indicate that ankyrin-G stabilizes EGFR in attached (HMLE) and detached (MCF10a) cells.

In cotransfection experiments, we found RIP1 and TRADD as ankyrin death domain interacting partners. Ankyrin-G interaction was confirmed in mammalian cells (HMLE and MCF10a cells). RIP1 co-operated with ankyrin-G induced apoptosis. The TRADD and ankyrin-G interaction couldn't be confirmed in co-immunoprecipitation experiments. Either overexpression or downregulation of TRADD enhance anoikis sensitivity, possibly by affecting the TNF signaling response. The TRADD overexpressing cells have dramatically low level of ankyrin-G, suggesting possible effect on protein stability, due to increased apoptotic events. Ankyrin-G seems to be a very complex protein, regulating various cellular processes. 


\section{CHAPTER 4}

NRAGE, AN ANKYRIN-G INTERACTING PROTEIN SUPPRESSES ANOIKIS Introduction

NRAGE (Neurotrophin Receptor-interacting mAGE protein) is a melanoma antigen (MAGE) family protein, identified through interaction with neutrotrophin receptor p75-NTR. It is also known as MAGE-D1 and Dlxin1 (DIx5 interacting protein). It interacts with the ZU5 domain containing protein, Unc5H1 (netrin receptor) and induces apoptosis in PC12 cells (Williams et al., 2003). Consistent with its role in neuronal apoptosis, the NRAGE knockout mouse showed defects in developmental apoptosis, quite similar to the p75 knockout mouse.

NRAGE also interacts with transcription factors such as Dlx5, Msx1/2, and Che1 to modulate their function. Interacting proteins at the cell membrane as well as in the nucleus require a tight regulation in nucleocytoplasmic transport. NGF treatment accumulated NRAGE at the cell membrane, whereas co-transfection with Msx2 promoted nuclear localization (Matsuda et al., 2003; Salehi et al., 2000).

There are reports that implicate the role of NRAGE in tumorigenesis but the exact mechanisms are not reported (Chu et al., 2007; Du et al., 2009). In this study, we have identified a novel cytoskeletal (ankyrin-G) and a transcription repressor (TBX2) as NRAGE interaction partners. These interactions have been characterized for their roles in regulation of nucleocytoplasmic transport, anoikis and anchorage independent growth in epithelial cells and the cells which have undergone epithelial-mesenchymal-transition (EMT). NRAGE functions differently in the nucleus and cytoplasm affecting transcription regulation or adherens junction kinetics. 
Results

Ankyrin-G interacts with MAGE family protein NRAGE: NRAGE has previously been shown to interact with the netrin receptor Unc5H1, a protein known to contain the ZU5-UPA-Death domain supermodule (Wang et al., 2009). This supermodule is conserved in other proteins such as PIDD (p53 induced death domain protein) and ankyrins (ankyrin-R, ankyrin-B, and ankyrin-G). Based on the Unc5 and NRAGE interaction, we tested the interaction between ankyrin-G and NRAGE. Experiments performed with mammary epithelial cell (MCF10a and HMLE) lysates suggested that ankyrin-G and NRAGE form a complex in cells (Fig.1).

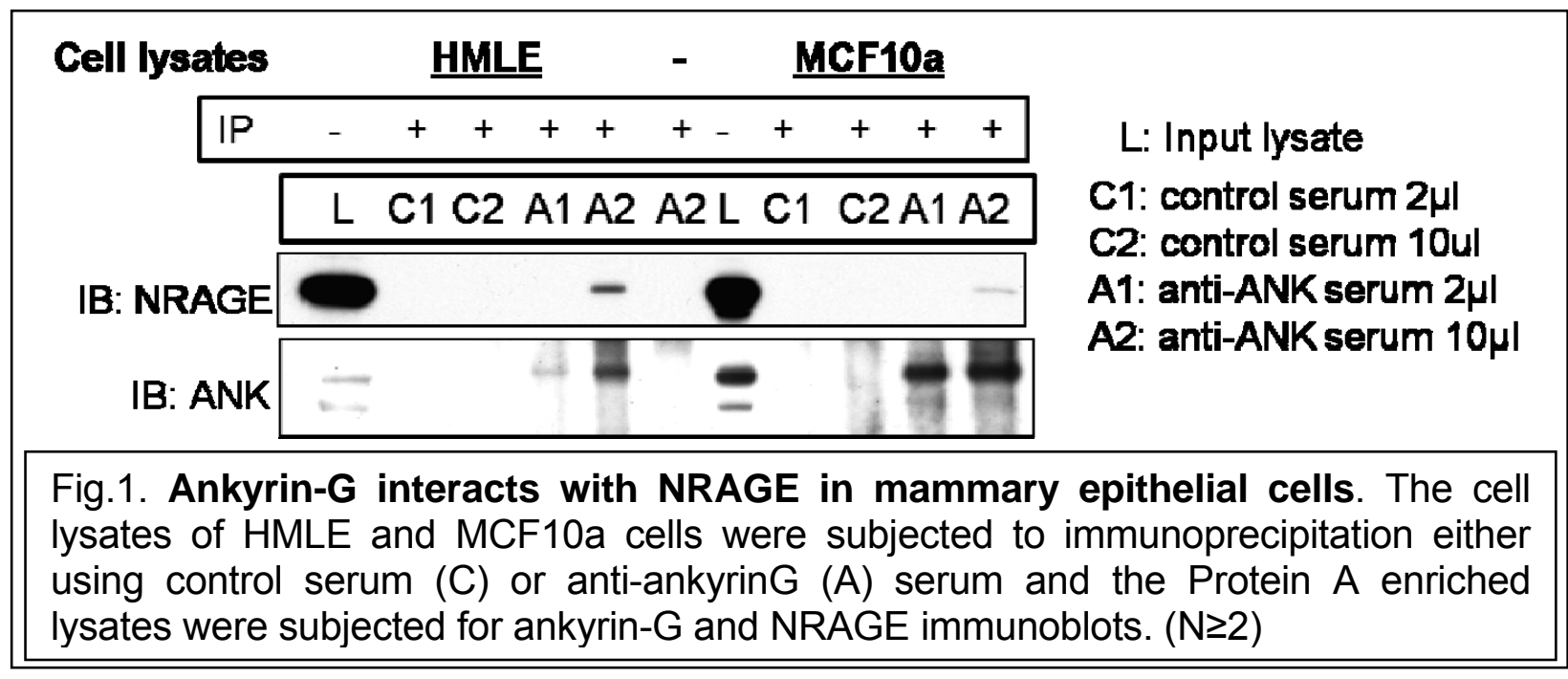

The specificity of ankyrinG-NRAGE interaction was tested using transient knockdown approach. We knocked down NRAGE or ankyrin-G in HMLE and MCF10a cells and the cell lysates were used for ankyrin-G immunoprecipitation. The NRAGE knockdown abolished the ankyrinG-NRAGE interaction whereas ankyrin-G knockdown substantially affected immunoprecipitation efficiency (fig.2). This observation confirmed that the interaction between NRAGE and ankyrin-G is specific. 


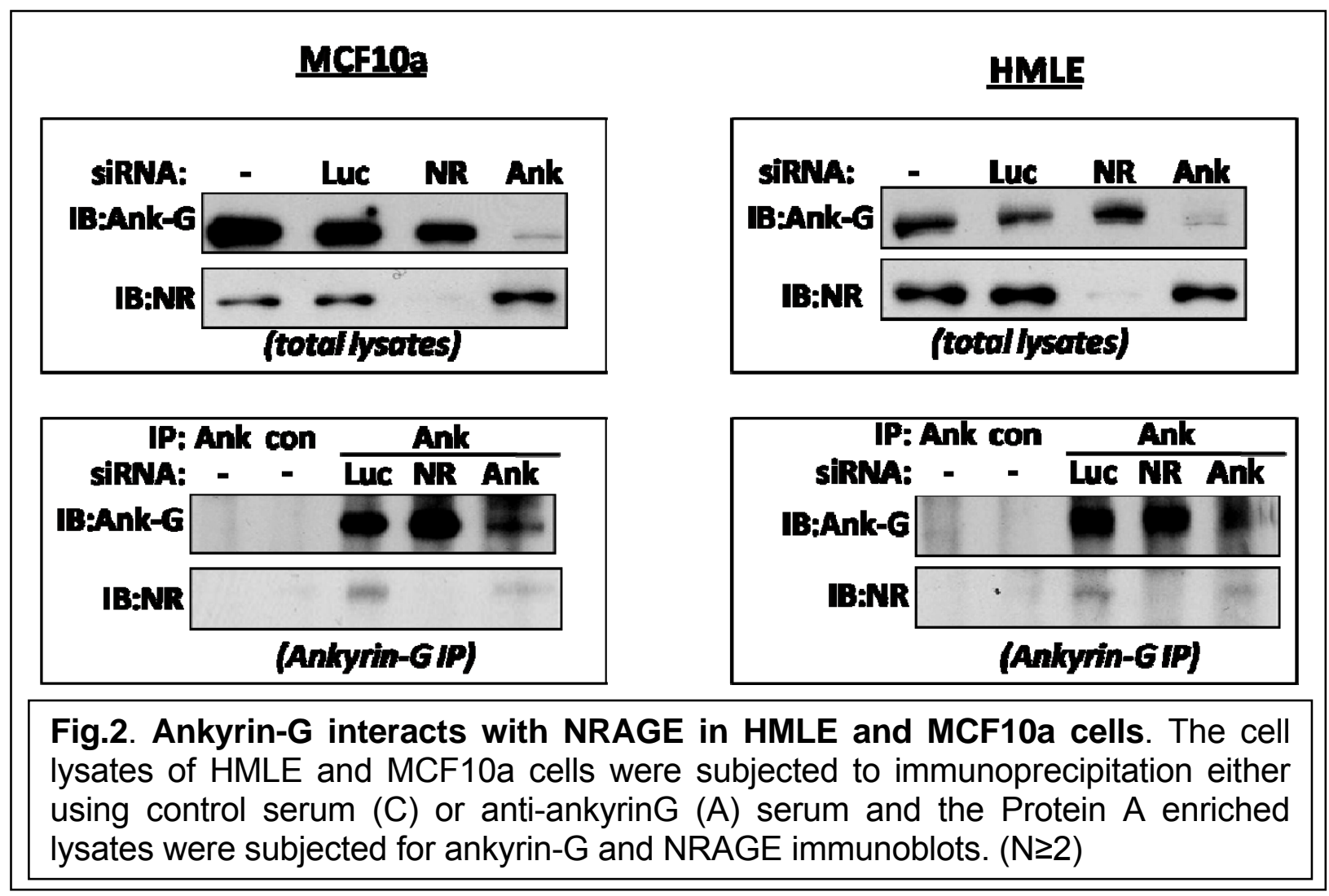

We also tried to co-immunoprecipitate NRAGE and blot for ankyrin-G but few existing ankyrin-G antibodies recognized non-specific bands which interfered with the real ankyrin-G band on immunoblots. To overcome this limitation, we stably expressed retroviral ANK-105 variant (containing HA-tag) in MCF10a cells and the cell lysates were used for NRAGE co-immunoprecipitation. The experimental data suggested that NRAGE could immunoprecipitate the exogenous ANK-105 (fig.3).

\begin{tabular}{|c|c|c|c|c|}
\hline \multirow{2}{*}{\multicolumn{2}{|c|}{ MCF10a cells }} & \multicolumn{2}{|c|}{ MCF10a/ANK-106 lysate } & \multirow{5}{*}{$\begin{array}{l}\text { Fig.3. NRAGE } \\
\text { immunoprecipitates } \\
\text { expressed exogenous ANK-105 in } \\
\text { MCF10a cells. Left panel showing the } \\
\text { immunoblot of the cell lysates } \\
\text { expressing HA-ANK105. The cell } \\
\text { lysates from MCF10a/ANK105 cells } \\
\text { were immunoprecipitated with NRAGE } \\
\text { or IgG control and blotted for NRAGE } \\
\text { and anti-HA antibodies. }\end{array}$} \\
\hline & & & $+\quad+\quad-$ & \\
\hline ANK-105 & + & IP: & NR IgG NR & \\
\hline IB: HA & $=$ & IB: HA & - & \\
\hline IB: NR & & IB: NR & - & \\
\hline
\end{tabular}


Characterization of domains requirement for NRAGE and ankyrin-G interaction: Ankyrin-G and NRAGE interacted in co-immunoprecipitation experiments (MCF10a and HMLE cell lysates). We further wanted to know the domain/s required for these interactions. Previously reported interaction indicated that the ZU5-UPA-DD supermodule of Unc5H1 was required for NRAGE interaction. Two of the transcript variants of ankyrinG, ANK-105 and ANK-120, contain these domains which otherwise lack the membrane binding domain (consist of 24 ankyrin repeats, present in membrane localized large ankyrin-G isoforms). Exogenous ANK-105 expressed in MCF10a cells could be immunoprecipitated by NRAGE (fig.3). The other ankyrin isoform is ANK-120, which differs with ANK-105 for the presence of a highly acidic insert within the regulatory Cterminal domain. We compared the splice variants of ankyrin (ANK-105 and ANK-120) for NRAGE interaction in co-transfection experiments. The mammalian expression construct for GST or GST-NRAGE was co-transfected with HA-ANK105 or HA-ANK120 in 293T cells, lysates were subjected to glutathione sepharose pulldown and enriched lysates were processed for HA and GST immunoblots. Both of the ankyrin construct products were pulled down with NRAGE whereas GST alone failed to do so (fig.4). The pulldown data suggested that the ANK-105 variant binding affinity with NRAGE was higher compared to ANK-120, indicating the possible role of C-insert in interfering with the ankyrinG-NRAGE interaction. Based on this information, we used ANK-105 constructs for screening the NRAGE domain required for interaction. 


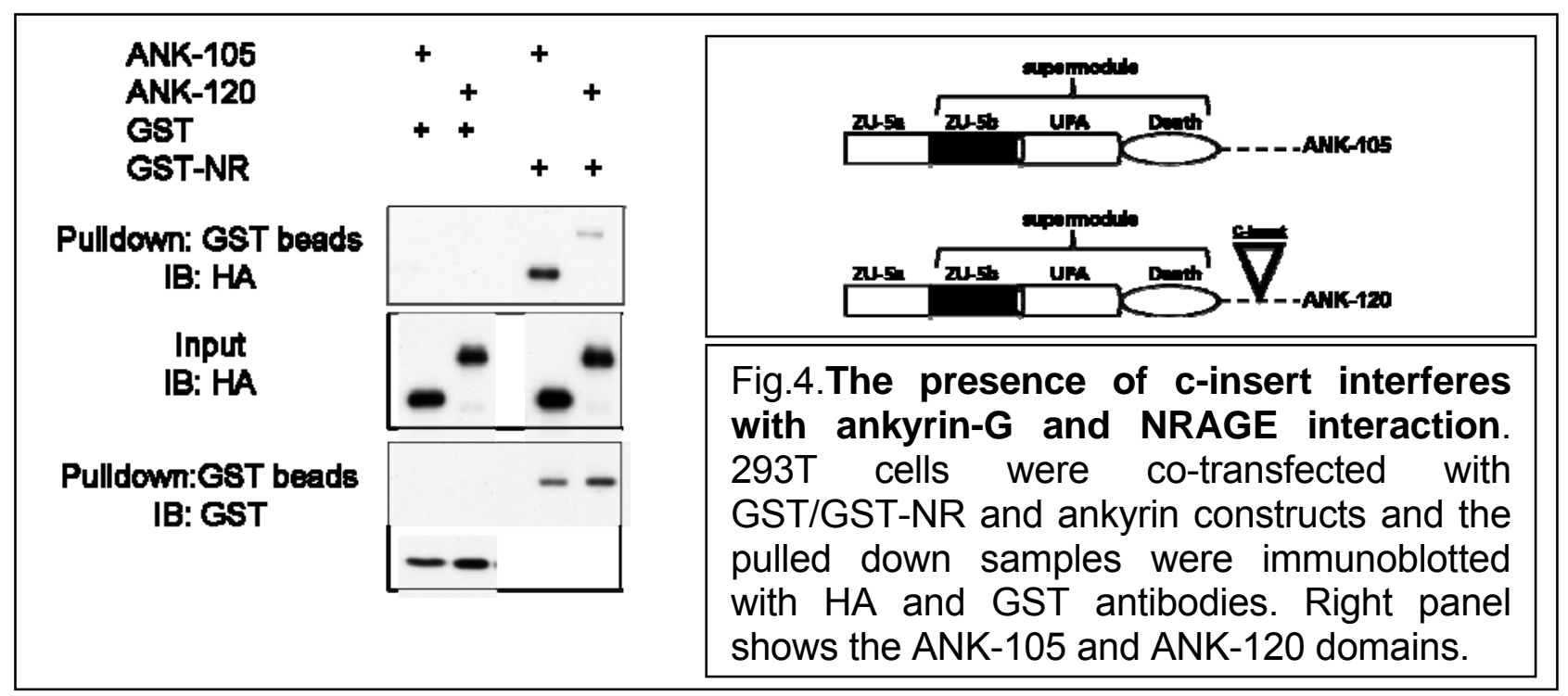

To find out the NRAGE domain required for ankyrin-G interaction, we designed various domain deleted constructs of NRAGE as a fusion product of GST. The various NRAGE constructs were co-transfected with HA-ANK105, and lysates were subjected to glutathione sepharose pulldown. The enriched lysates were subjected to anti-HA and anti-GST blots. The data suggested that the MAGE domain and C-terminal domains of NRAGE were required for ankyrin-G interaction (fig.5). Deletion of the MAGE domain and C-terminal domain of NRAGE dramatically reduced the ankyrin-G interaction.

To investigate the requirement of ankyrin-G domain/s for NRAGE interaction, the human ankyrin-G construct was used. Considering that ankyrin- $G$ is a large molecular weight protein ( $210 \mathrm{kd})$ and doesn't require its membrane binding domain for NRAGE interaction (fig. 3, 4, and 5), we designed an ankyrin-G construct that lacked the membrane binding domain (MBD) and first tested if ZU5 domains (ZU5-A and ZU5-B) were required for NRAGE interaction. 


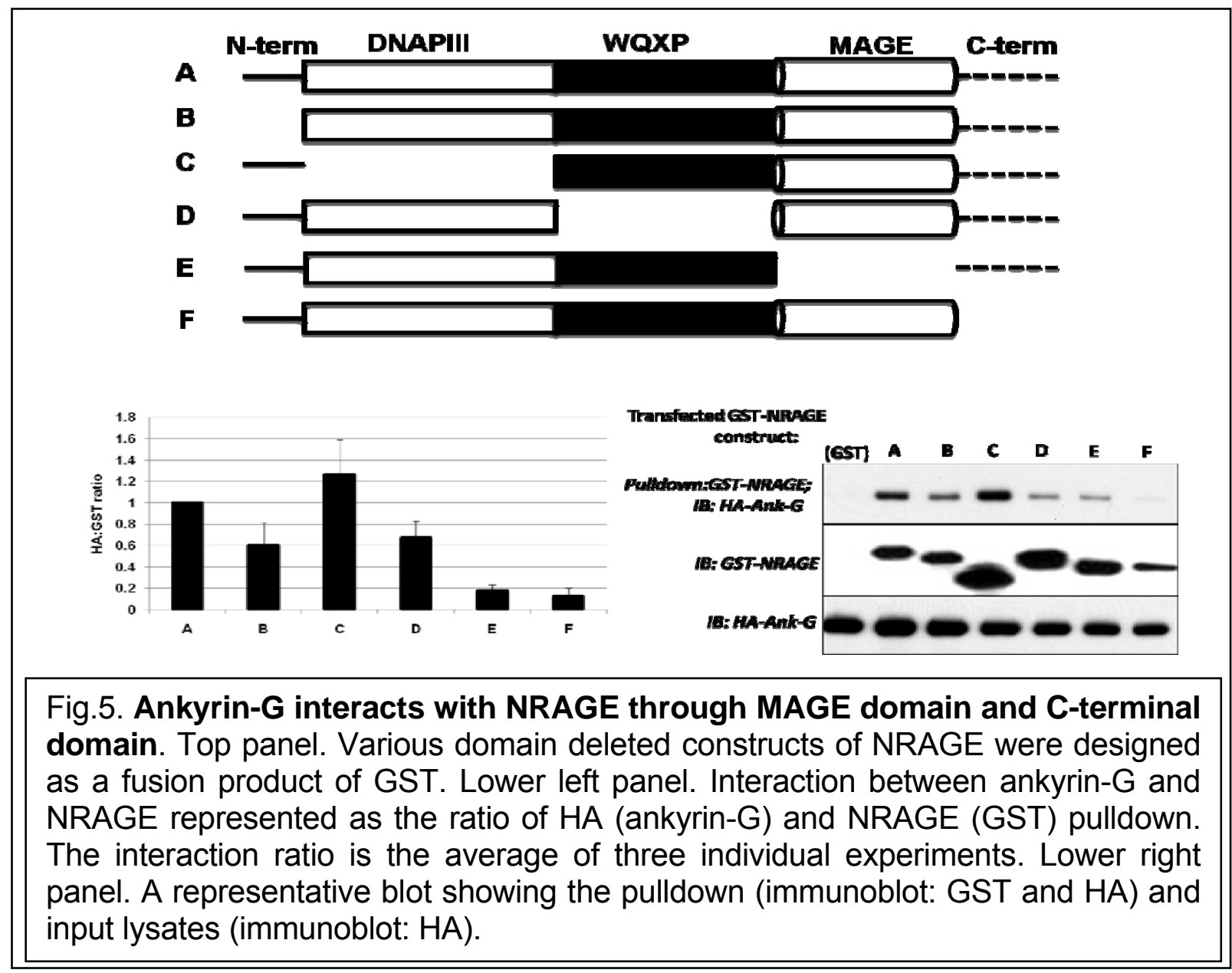

The constructs used for this experiment lacked MBD and either of ZU5-A or ZU5-B domains. These constructs were co-transfected in 293T cells either with GST or GSTNRAGE, and cell lysates were enriched using glutathione sepharose beads. The immunoblot data suggested that deletion of either of the ZU5 domains didn't affect NRAGE interaction (fig.6). Surprisingly, deletion of the ZU5-A domain enhanced expression and interaction with NRAGE. In subsequent experiments, we used ankyrin constructs lacking both the membrane binding domain as well as ZU5-A domain. The other set of constructs designed either lacked the UPA domain or had only the UPA domain and was tested for NRAGE interaction. 


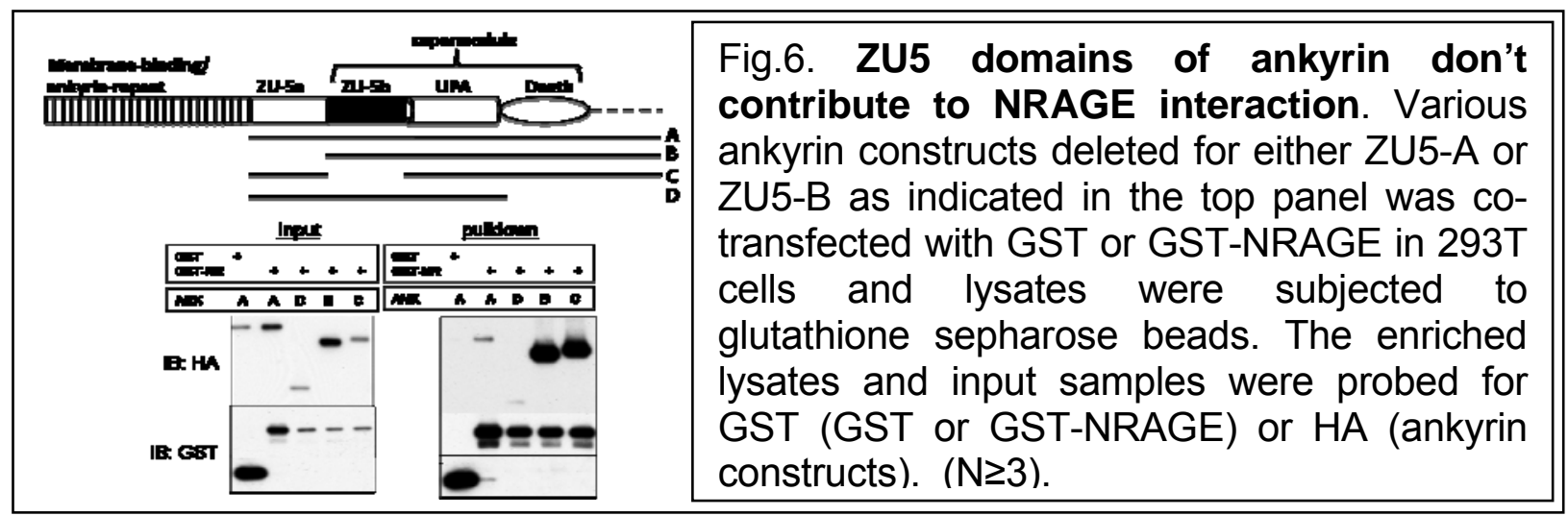

The co-transfection experiments followed by glutathione sepharose pulldown suggested that the loss of the UPA domain dramatically reduced the interaction. The UPA domain alone was sufficient for NRAGE interaction (fig.7). The UPA domain is part of the supermodule containing ZU5b-UPA-DD in ankyrin-G, unc5H1 and PIDD proteins. Interaction with NRAGE is the first reported function of any UPA domain. It is yet to be verified if other UPA domains (specially the previously reported unc5 $\mathrm{H} 1$ interaction with NRAGE) interact with NRAGE. Its biological significance is yet to be determined. Based on these data, we hypothesize that the UPA domain might sequester NRAGE in ankyrinG based cytoskeleton.

NRAGE affects Ankyrin-G function and epithelial polarity: NRAGE interacts with ankyrin-G and might affect ankyrin-G cellular function. We tested the role of NRAGE on ankyrin-G membrane localization. MDCK cells were transfected either with ankyrin-G, NRAGE or ankyrin-G/NRAGE. Cells were grown to confluency and coimmunofluorescence (using HA for NRAGE and ankyrin-G) was performed. 

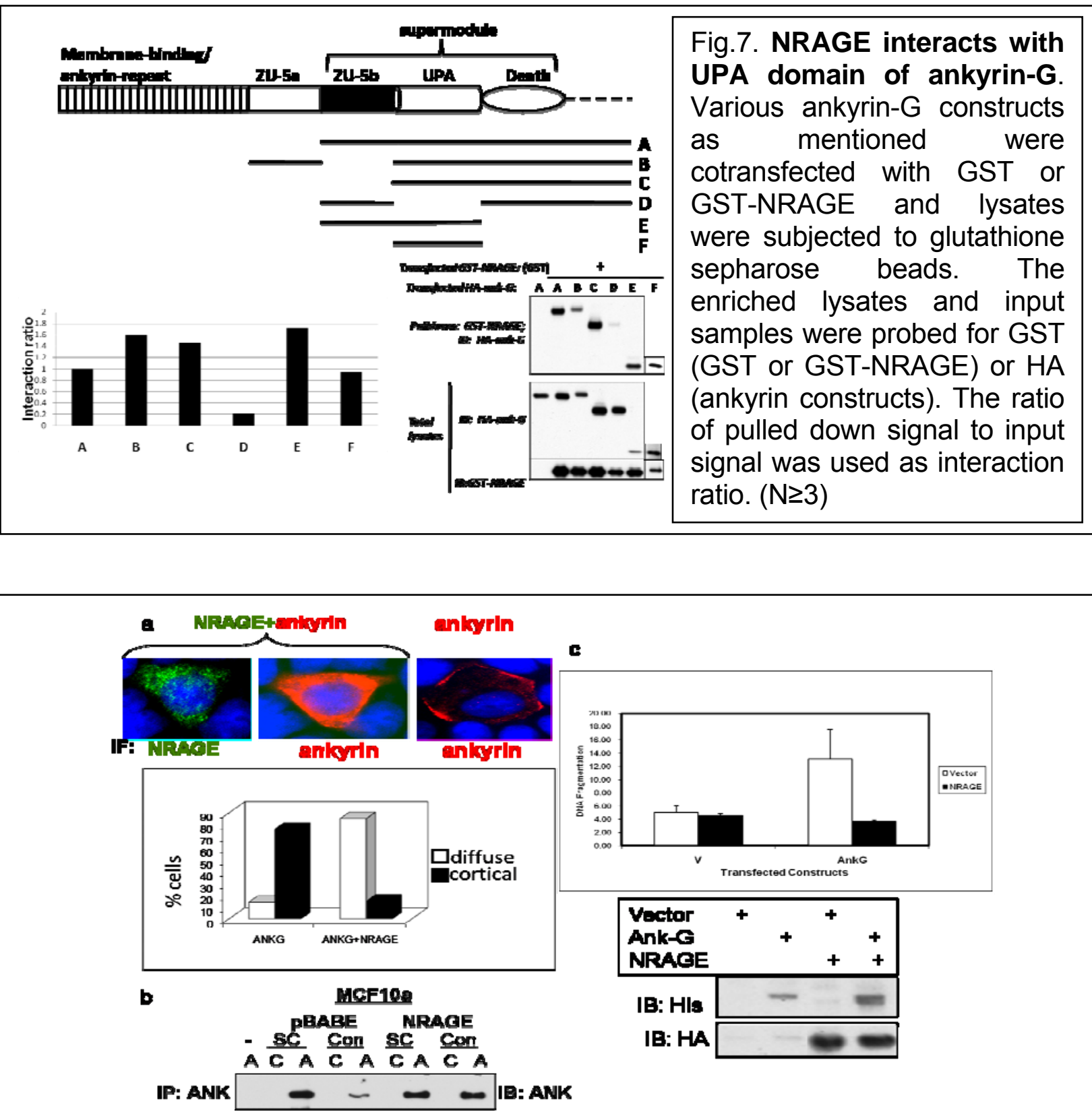

Fig.8. NRAGE affects cellular and biochemical characteristics of ankyrin-G. (a.) NRAGE affects ankyrin-G localization. The rat ankyrin-G constructs were transfected with vector or NRAGE (MDCK). The fixed cells were stained for ankyrin$G$ and HA. $(N \geq 3)$ (b.) NRAGE affects ankyrin-G solubility in confluent cells. Subconfluent (SC) and confluent (C) MCF10a (-/+NRAGE) cell lysates were immunoprecipitated either with control (C) or ankyrin-G (A) antibodies and blotted for ankyrin-G. (c) The H1299 cells were transfected with vector, NRAGE, ankyrin-G or NRAGE/ankyrin-G and lysates were subjected for immunoblots (anti-HA and anti-His) and DNA fragmentation ELISA. The DNA fragmentation values were normalized with relative protein amount. 
The data suggested that ankyrin-G localized to the membrane whereas NRAGE co-transfection dramatically shifted ankyrin-G to cytoplasm (fig. 8a). Ankyrin-G is resistant to detergent solubility in association with cytoskeleton in confluent cells. In subconfluent cells, ankyrin-G was more soluble to detergent compared to confluent cells, possibly because of difference in cell-cell contact. The subconfluent and confluent cell culture (MCF10a-/+ NRAGE) lysates were immunoprecipitated and immunoblotted with ankyrinG antibody.

The immunoprecipitation data suggested that ankyrin-G couldn't be solubilized effectively in confluent cells compared to subconfluent cells in control cells. The cells expressing NRAGE showed no obvious difference in detergent solubility, in subconfluent and confluent cells (fig. 8b). These data suggest that NRAGE expression results in increased detergent solubility of ankyrin-G, probably by affecting membrane localization which is also supported by the immunofluorescence data (fig. 8a). This observation suggests that NRAGE might affect membrane properties of ankyrin-G and is otherwise required for membrane biogenesis and other related membrane functions (Kizhatil and Bennett, 2004). We have previously observed that the overexpression of ankyrin-G induces apoptosis. We tested the effect of NRAGE on ankyrin-G induced apoptosis. The co-transfection experiment performed in $\mathrm{H} 1299$ and cos7 cells suggested that NRAGE suppresses ankyrin-G induced apoptosis (fig.8c.). 


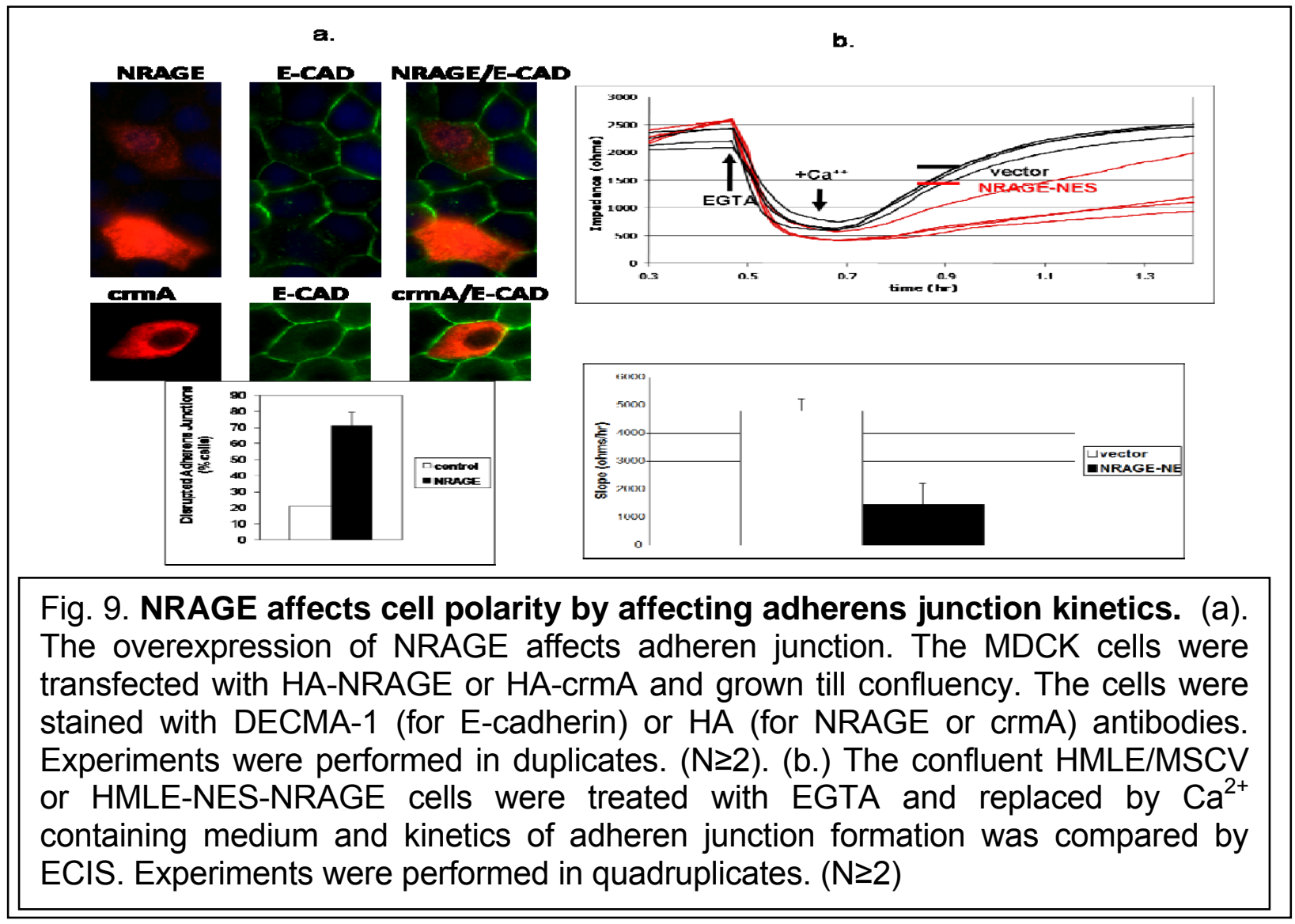

Ankyrin-G is part of the E-cadherin complex, controls membrane biogenesis and regulates E-cadherin membrane localization. Based on NRAGE's role on ankyrin-G functions, we hypothesized that NRAGE might affect E-cadherin localization at the adherens junction. To test E-cadherin localization, MDCK cells were transfected either with vector or NRAGE and grown to confluency. Fixed cells were stained for E-cadherin and HA (NRAGE). The E-cadherin staining patterns (either intact or diffuse) were scored. NRAGE overexpression resulted in diffuse E-cadherin localization (fig.9a). The overexpression assays are an artificial system and we wanted to test E-cadherin functions in a more physiological context using ECIS (electric cell impedence sensing) for testing adherens junction kinetics. Cells were treated with EGTA (a known chelator for $\mathrm{Ca}^{2+}$, which destabilizes E-cadherin mediated adherens junction), and then the cells were 
replaced subsequently with $\mathrm{Ca}^{2+}$ containing medium, resulting in the formation of adherens junction. Adherens junction kinetics (cell impedence as readout) was compared between control and NRAGE expressing HMLE cells. The data suggested that NRAGE overexpression ( 2-3 folds) significantly affected the adherens junction kinetics and showed impaired ability to form cell junctions (fig.9b). These data suggest that NRAGE affects adherens junction kinetics and hence epithelial cell polarity.

NRAGE is an anoikis suppressor: NRAGE suppressed ankyrin-G induced apoptosis and affected the adherens junction kinetics. As an apoptosis suppressor, we hypothesized that NRAGE might play a role in anoikis (apoptosis suppressed by cellextracellular matrix interaction), an apoptosis known to suppress metastasis (Frisch and Screaton, 2001). To test the hypothesis, we used the transient knockdown experiments in mammary epithelial cells (MCF10a) and endothelial cells (iHUVEC). Transient knockdown of NRAGE enhanced anoikis (caspase activity assay as an apoptosis readout) in both cell types, MCF10a and iHUVEC cells, suggesting NRAGE as a possible anoikis suppressor.

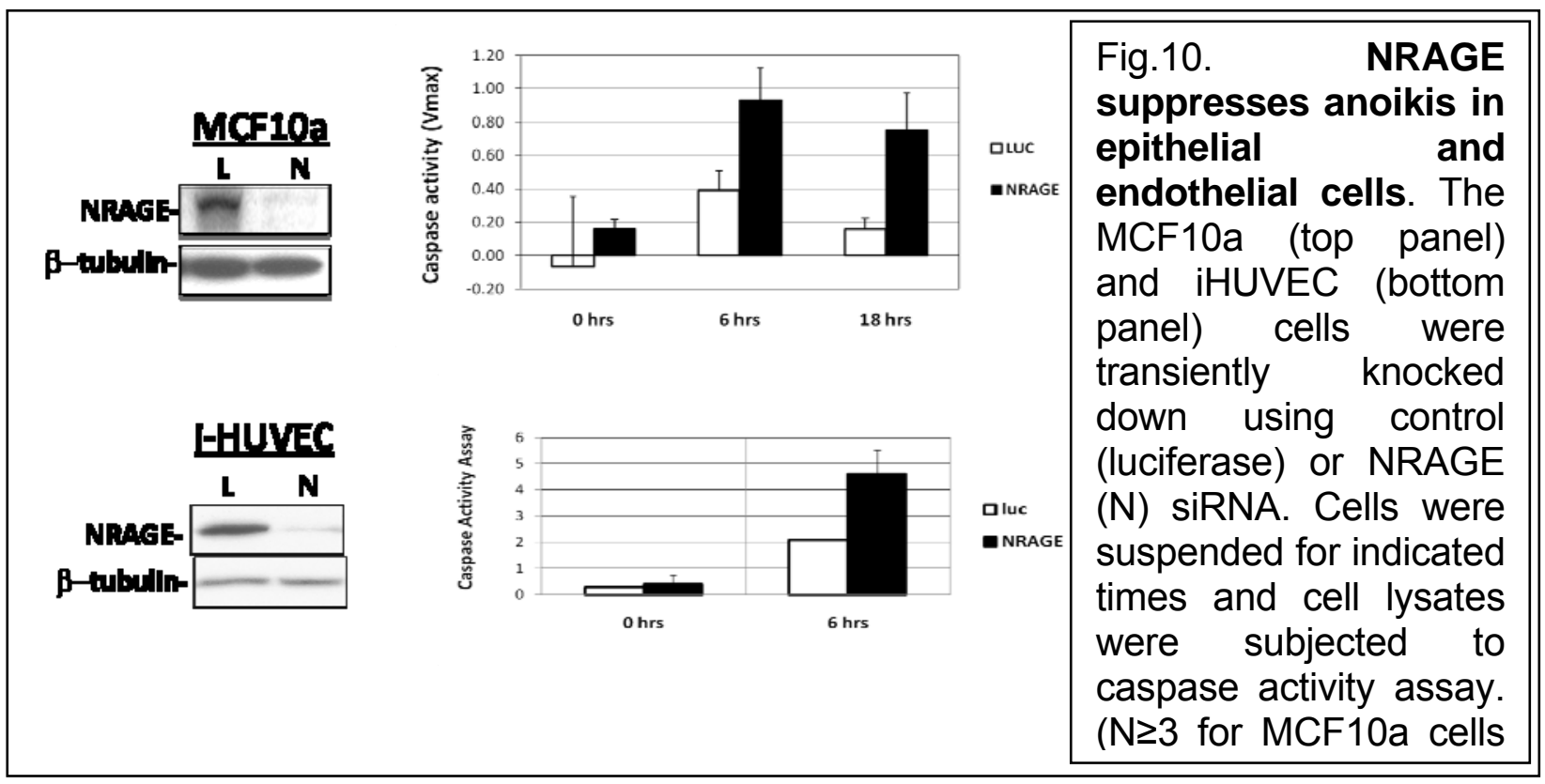


We also explored other well characterized epithelial cell lines such as MDCK and HMLE. We stably expressed human NRAGE using retroviral infection followed by antibiotic selection. The NRAGE expressing cells were confirmed for NRAGE overexpression ( 2-3 fold expression compared to MSCV control). We further compared control and NRAGE expressing cells for anoikis. NRAGE expression suppressed anoikis in MDCK cells (data not shown). In HMLE cells we grew cells to confluency and an equal amount of cells were suspended in rotating tubes to avoid aggregation and lysates collected at different suspension time and assayed for caspase activity (fig.11a). The data suggested that NRAGE expressing cells were partially resistant to anoikis. To further test if the anoikis sensitivity in HMLE cells were a result of NRAGE overexpression, we knocked down NRAGE in cells with exogenous NRAGE. The knockdown of NRAGE restored anoikis sensitivity in partially resistant HMLE+NRAGE cells (fig.11b). These data suggest that over-expression of NRAGE makes cells resistant to anoikis whereas transient knockdown reverses the anoikis sensitivity in partially anoikis resistant cells.

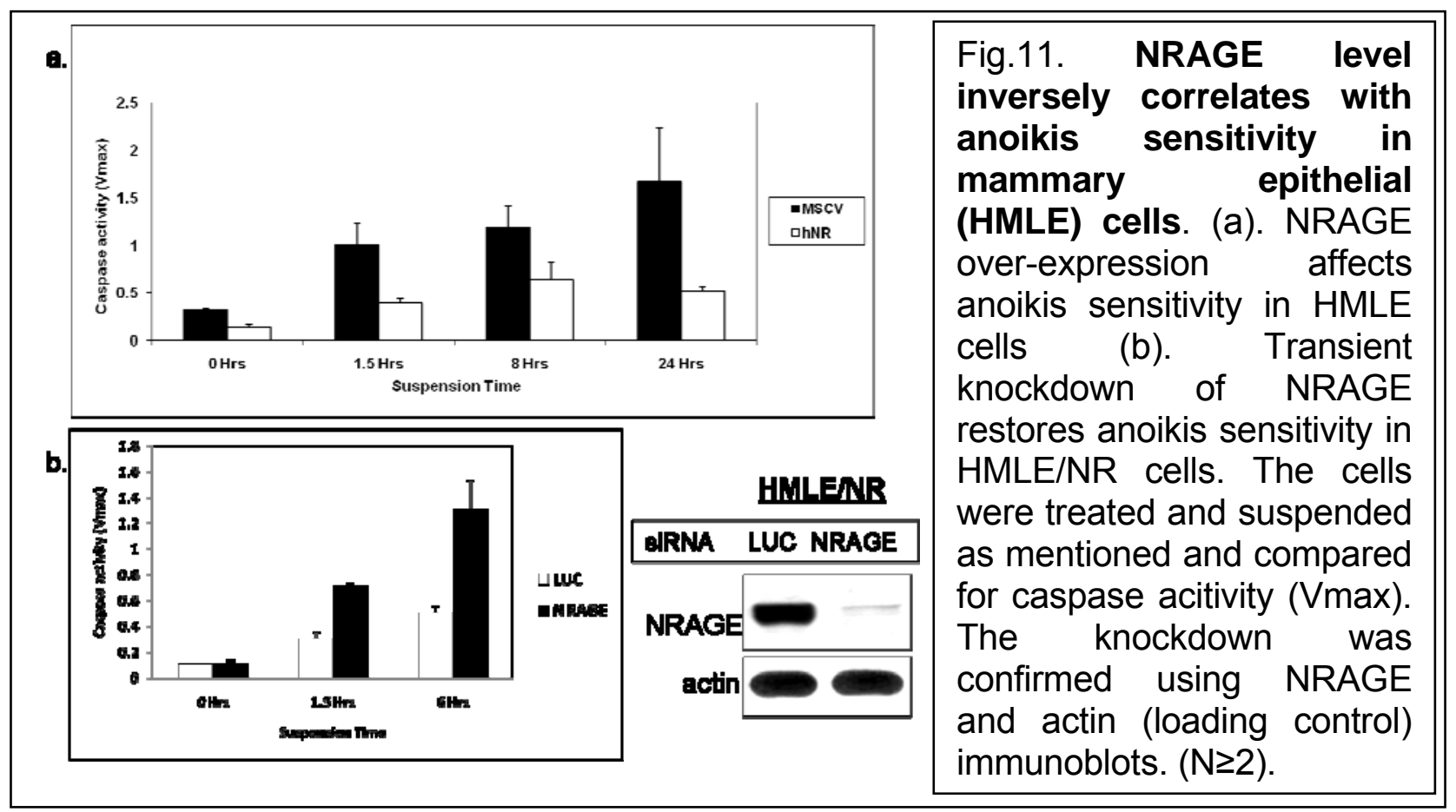


We also tested the effect of transient knockdown or overexpression of NRAGE on anoikis using DNA fragmentation as apoptosis readout. As observed with caspase activity assay, NRAGE knockdown or overexpression affected DNA fragmentation in a similar fashion. The overall data suggest that NRAGE is an anoikis suppressor. Anoikis is a safeguard mechanism against tumor metastasis and its suppression can result in increased tumor metastasis and carcinoma. Tumor progression affects anoikis sensitivity and we tested if there was any correlation between NRAGE expression with cell type and cancer stage.

NRAGE is overexpressed in Breast cancer cell lines and cells undergone EMT: NRAGE was characterized as an anoikis suppressor and predicted to be involved with tumor progression. We tested the expression level of NRAGE in various tissue lysates (comparing normal and tumor samples), breast cancer cells and cells which have undergone EMT (epithelial-mesenchymal-transition). We first compared the protein level of NRAGE in various normal and tumor tissue samples (blot obtained from G-Biosciences Inc). The immunoblot data suggested that NRAGE was over-expressed in tumor samples of uterus, breast, ovary and Head/Neck tissues (fig.12a). We also compared a few breast cancer cells with the normal mammary epithelial cell line MCF10a for NRAGE expression. NRAGE was upregulated in all the breast cancer cells (MCF7, MDA-MB-231, Au565 and BT474) tested (fig.12b). 


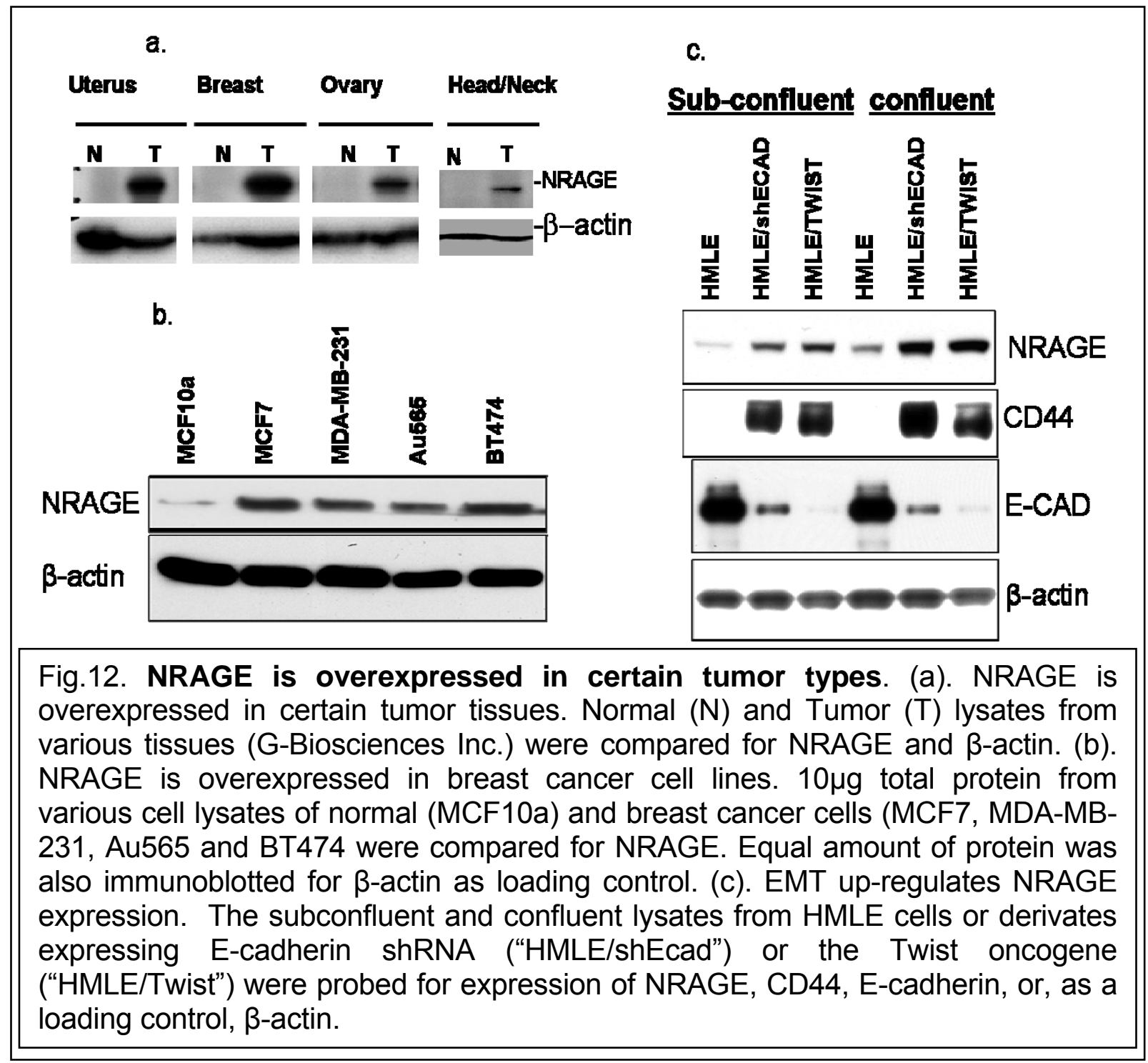

All tumor types have been shown to undergo epithelial mesenchymal transition (EMT). We wanted to test if cells which have undergone EMT might affect NRAGE expression. We utilized a well characterized mammary epithelial cell line, HMLE and its EMT counterpart HMLE + shECAD (stable knockdown of E-cadherin) and HMLE + Twist (stable expression of Twist oncogene). These cells have previously been characterized for anoikis resistance (Onder et al., 2008). The induction of EMT in this system by either method induced NRAGE level, which correlated with the induction of CD44 and repression of E-cadherin (fig.12c). Preliminary observations comparing the protein level in 
tumor samples, breast cancer cells and during EMT induction suggests that NRAGE is upregulated during EMT and tumor progression. The NRAGE upregulation in EMT cells was confirmed through quantitative PCR, suggesting the involvement of transcriptional regulation (Frisch SM, unpublished observation). This study was continued with testing the expression level of NRAGE in breast cancer and lung cancer patient samples (48 samples each). NRAGE transcript was found to be upregulated in all stages (dramatic at stage I and III) of breast cancer. Among lung cancer patients, NRAGE upregulation was found to be stage dependent (Frisch SM, unpublished observation).

We extended our study by comparing the NRAGE protein level in various carcinoma samples which included colon carcinoma, melanoma and prostate cancer samples using Automated Quantitative Analysis (AQUA), a quantitative immunofluorescence technology using two color fluorescence imaging (Camp et al., 2002). NRAGE was found to be upregulated in colon carcinoma, metastatic melanoma, and prostate cancer samples (unpublished data in collaboration with Elizabeth Killiam and David Rimm at Yale University, CT).

NRAGE upregulation during EMT and variety of cancer cells as well as its function as anoikis suppressor suggest that NRAGE is a pro-tumorogenic protein and might support the tumor progression primarily by suppressing anoikis.

NRAGE suppresses anoikis and promotes anchorage independent growth in EMT cells: EMT cells acquire resistance to anoikis and therefore promote tumor metastasis. The EMT cell models of mammary epithelial HMLE (by stable knockdown of E-cadherin or Twist overexpression) cells were resistant to anoikis (Onder et al., 2008). Re- 
expression of mouse E-cadherin in HMLE + shECad cells restores sensitivity to anoikis resistance (Frisch SM, unpublished observation).

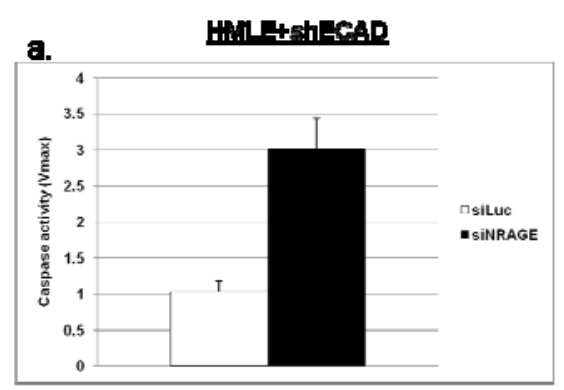

b.
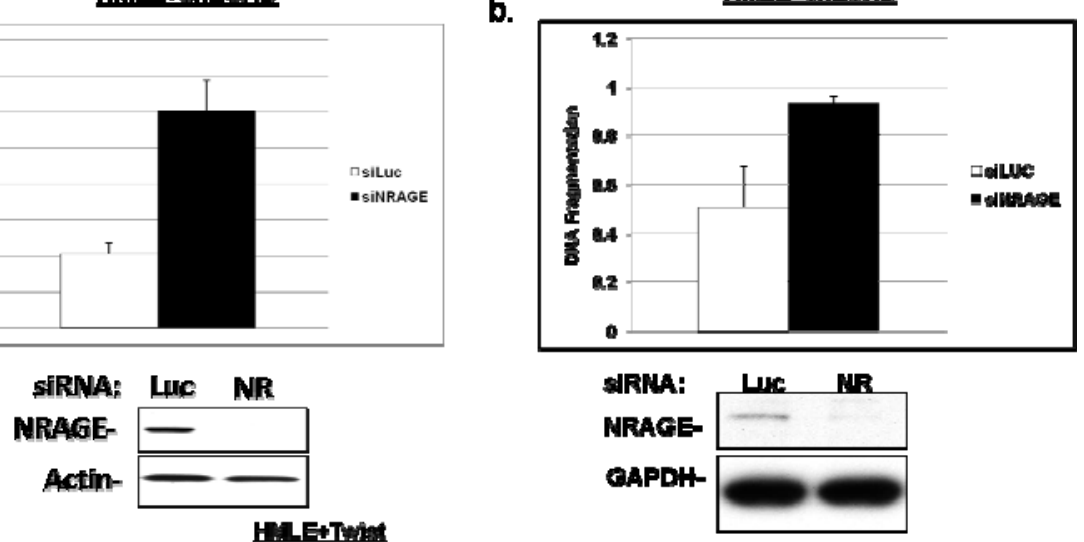

c.
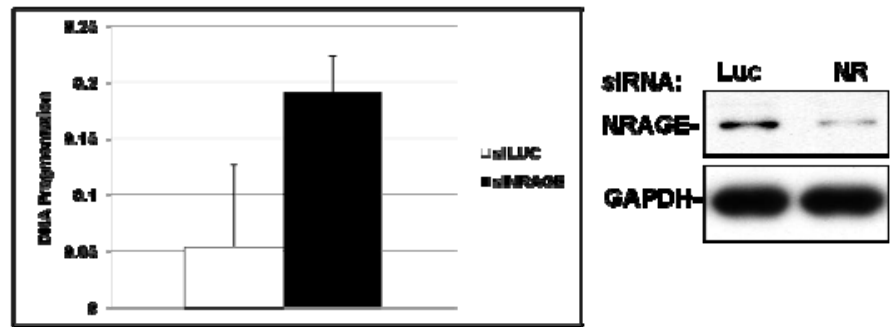

Fig.13. Transient knockdown of NRAGE promotes anoikis in EMT cells. HMLE + shECAD cells (a) and (b) and HMLE + Twist cells (c) were transfected with luciferase (Luc) or NRAGE siRNA and suspended cell lysates were processed for (a) caspase activity (Vmax) or (b) and (c) DNA fragmentation ELISA. The transient knockdown was confirmed by NRAGE (NR) immunoblot using actin or GAPDH as loading control. $(\mathrm{N} \geq 2)$

The EMT cells upregulated NRAGE compared to epithelial cells. We tested if NRAGE level correlates with anoikis resistance in EMT cells. To test the effect of NRAGE in EMT cells, we transiently knocked down NRAGE in HMLE+shEcad or HMLE+Twist cells, and cell lysates were assayed for DNA fragmentation or caspase activity (Vmax). The NRAGE knockdown enhanced the anoikis in EMT cells, with increased caspase activity (fig.13a) and fragmented DNA (fig.13b and 13c) as apoptosis readout. These knockdowns didn't affect the EMT, as far as morphology and E-cadherin expression 
pattern were tested (unpublished observation). These data suggest that the knockdown of NRAGE can partially restore anoikis sensitivity in EMT cell lines.

Anoikis suppression correlates with anchorage-independent growth in appropriate cellular context, when cell cycle regulations are bypassed. We compared the ability for HMLE, HMLE+shECAD and HMLE+Twist for anchorage independent growth. The cell types which have undergone EMT demonstrated anchorage independent growth in soft agar assay far more efficiently than parental HMLE cells. We transiently knocked down NRAGE in HMLE+shECAD and HMLE+Twist cells, followed by anchorage-independent growth assays on soft agar. The knockdown of NRAGE partially suppressed the anchorage independent growth of EMT cells (fig.14a). We also tested the effect of stable NRAGE knockdown in tumor cell line MDA-MB-435 for anchorage independent growth. Similar to previous observation in EMT cells, NRAGE knockdown suppressed anchorage independent growth in MDA-MB-435 cells (fig.14b). Our data indicate that NRAGE level correlates with anoikis suppression and anchorage-independent growth in EMT and tumor cells, suggesting its possible over-expression effect in tumor cells. NRAGE might promote anoikis suppression and anchorage independence growth in certain cellular contexts, perhaps by interaction with pro-tumorigenic genes. 
a
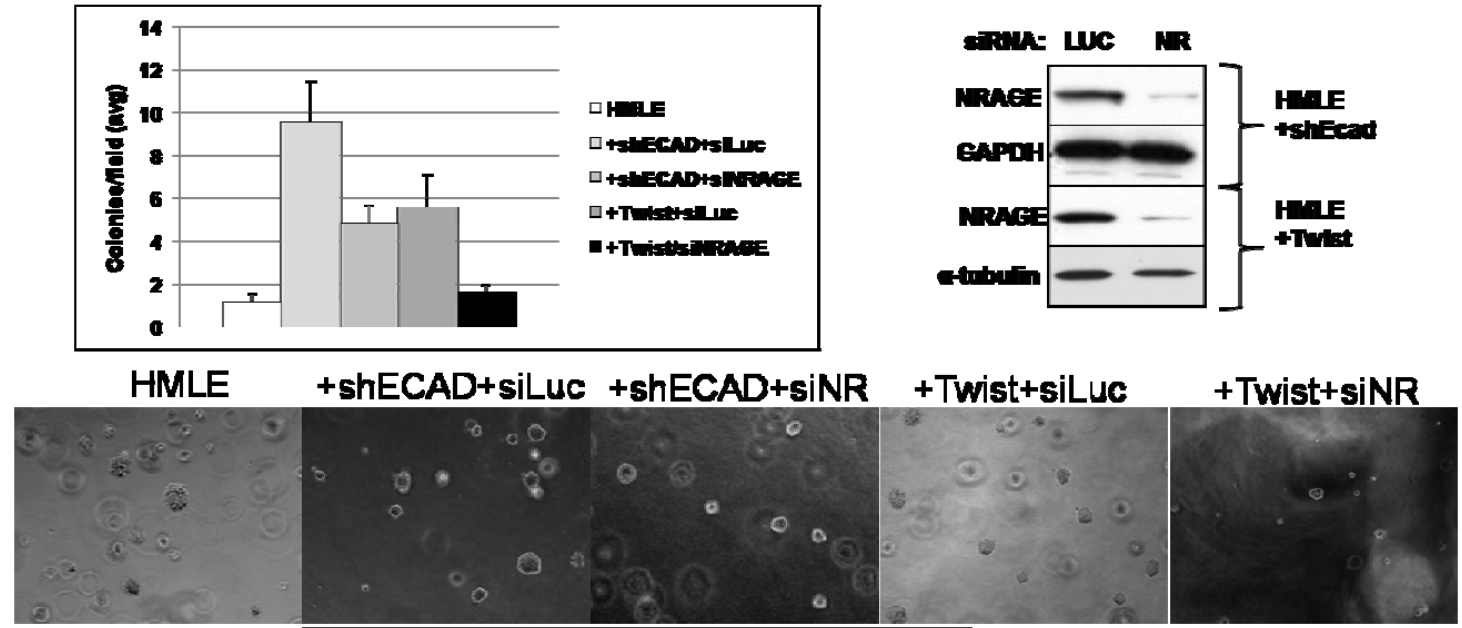

b

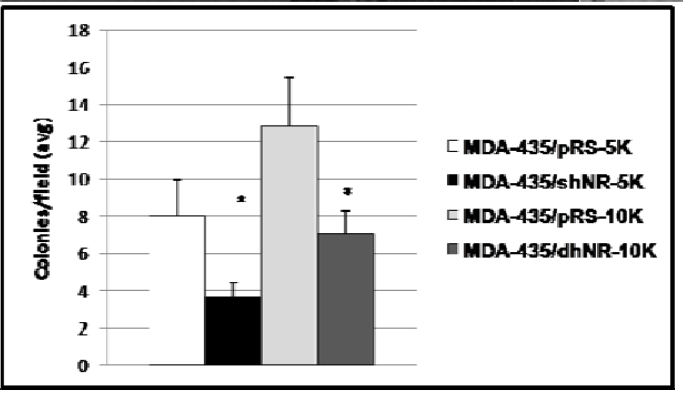

Fig.14. NRAGE promotes anchorage independent growth in EMT and tumor cells. (a) The HMLE+shECAD and HMLE+Twist cells were transiently transfected with luciferase or NRAGE siRNA and plated along with parental HMLE cells for soft agar colony assay. The cells were grown for 7-10 days and colonies from 6 wells were compared. The average colonies were normalized with colonies in normal plates. $(\mathrm{N}=2)$. (b) MDA-MB-435 cells were stably selected for control pRS or NRAGE shRNA expressing retroviral vectors and knockdown of NRAGE was confirmed. Either 5,000 $(5 \mathrm{~K})$ or $10,000(10 \mathrm{~K})$ cells were plated onto $0.7 \%$ low melting agarose containing DME medium (in 6 replicates) and colonies were compared after 7-10 days. Average colony numbers were normalized with colonies in normal wells. $(\mathrm{N}=2)$

NRAGE interacts with oncogenic transcription factor TBX2: There is various interaction partners reported to interact with NRAGE. But the effect on anoikis and anchorage independent growth can't be explained by any of these interactions. To identify the interacting partner/s which could possibly explain the NRAGE role in anoikis and anchorage independence growth, a yeast two-hybrid interaction was performed. The screen identified several candidate genes such as TBX2, KIAA0676, DAZAP2, DOCK6, 
and a previously reported ubiquitin ligase, Praja2 (Sasaki et al., 2002). TBX2 has previously been reported for promoting anchorage independent growth and protumorogenic activity. We decided to pursue this interaction in mammalian cells using coimmunoprecipitation experiments. The NRAGE-TBX2 interaction was confirmed in MCF10a and HMLE cells (fig.15). TBX2 antibody immunoprecipitation efficiency was very poor, so immunoprecipitation in the other direction couldn't be performed. These interactions have also been confirmed in HMLE+shECAD and HMLE+Twist cells (Frisch SM, unpublished).

\section{MCF10a}

Immunoprecipitation

\section{HMLE}

Immunoprecipitation

Cell Lysate

NR NR IgG TBX2 TBX2

IB: TBX2

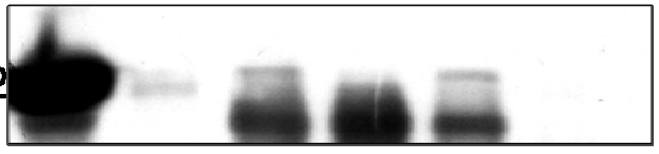

IB: NRAGE

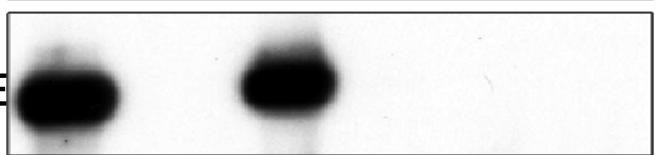

$\underline{N R} \quad \underline{\lg G} \quad \underline{N R}$

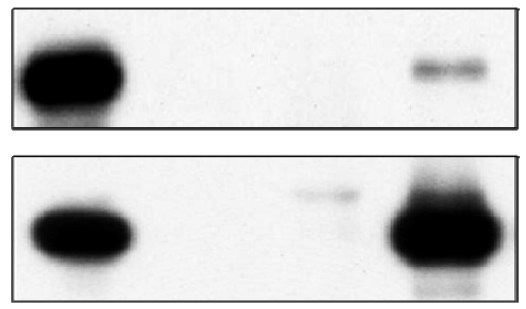

Fig.15. NRAGE interacts with TBX2 in MCF10a and HMLE cells. MCF10a (left panel) and HMLE (right panel) cell lysates were immunoprecipitated either with control rabbit IgG or rabbit NRAGE antibody. The immunoprecipitated samples were probed with TBX2 or NRAGE antibody. TBX2 antibody immunoprecipitation efficiency is very poor, though immunoprecipitate showed same band as NRAGE antibody (left panel).

To identify the NRAGE domain required for TBX2 interaction, NRAGE domain deletion constructs (GST tagged) were co-transfected with FLAG-TBX2 in 293T cells and the lysates were pulled down either using FLAG beads or glutathione sepharose followed by FLAG or GST/NRAGE immunoblots. The interaction data suggested that TBX2 interacts with c-terminal domain of NRAGE (fig.16). The c-terminal domain contains about 
120 amino acids with unknown function, though quite conserved among species (Chomez et al., 2001).

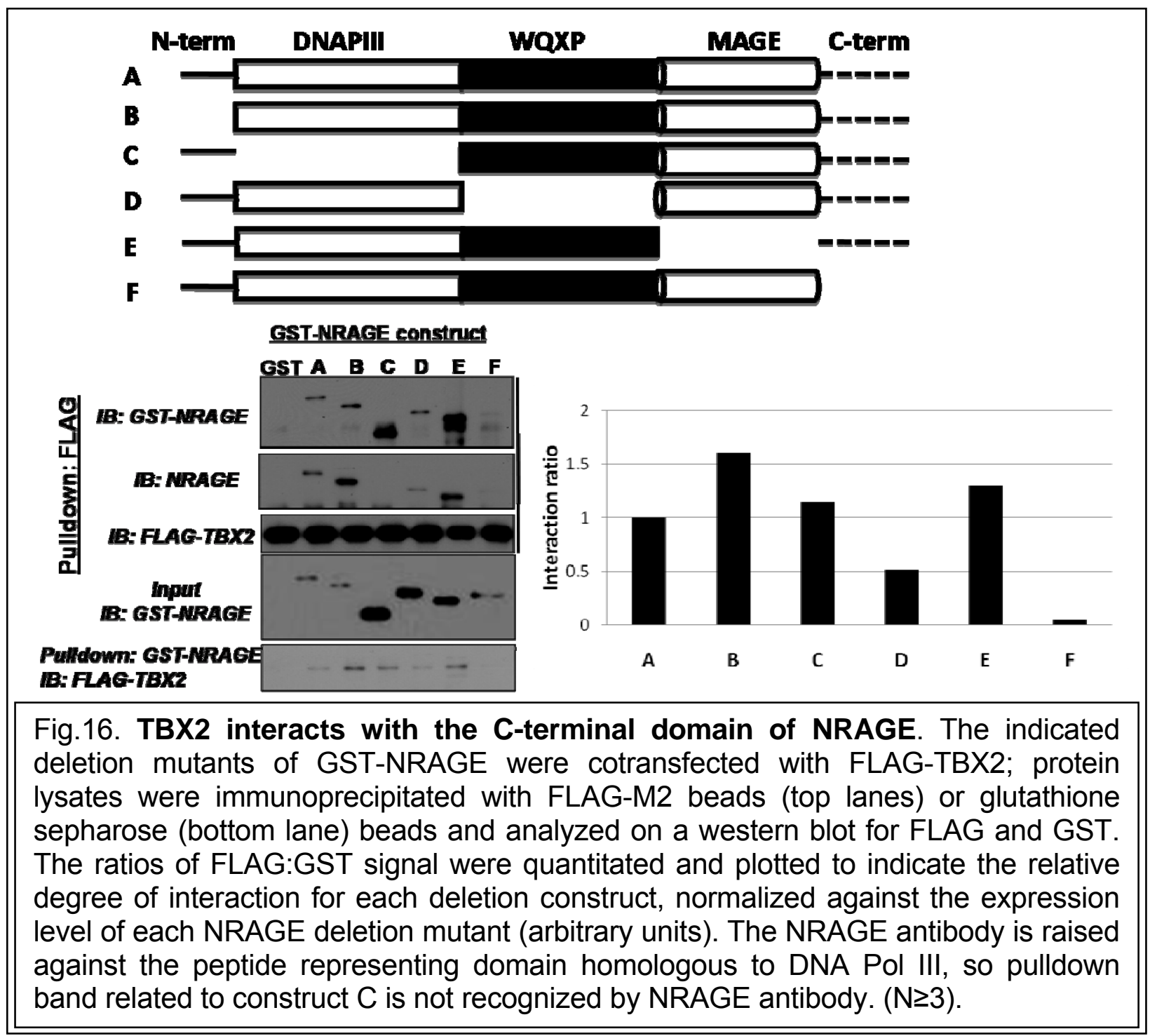

In the yeast 2-hybrid screen, there were many TBX2 clones identified as NRAGE interaction partners. The shortest cDNA containing clone found to have part of the repression domain $(\mathrm{RD})$ near the c-terminal domain. In pulldown experiments, the repression domain of TBX2 was sufficient to pulldown NRAGE whereas expression construct lacking repression domain failed, suggesting that TBX2 repression domain as 
NRAGE interaction domain. NRAGE interaction with the repression domain suggests a possible regulation in TBX2 dependent gene repression function.

TBX2 functionally co-operates with NRAGE: TBX2 is reported to have antiapoptotic as well as pro-tumorigenic activities such as counteracting cisplatin (DNA damaging agent) induced apoptosis (Davis et al., 2008). The TBX2 also promoted anchorage-independent growth in SW13 adenocarcinoma cells and mouse embryo fibroblast lacking retinoblastoma and its pocket proteins (Ismail and Bateman, 2009; Vormer et al., 2008).
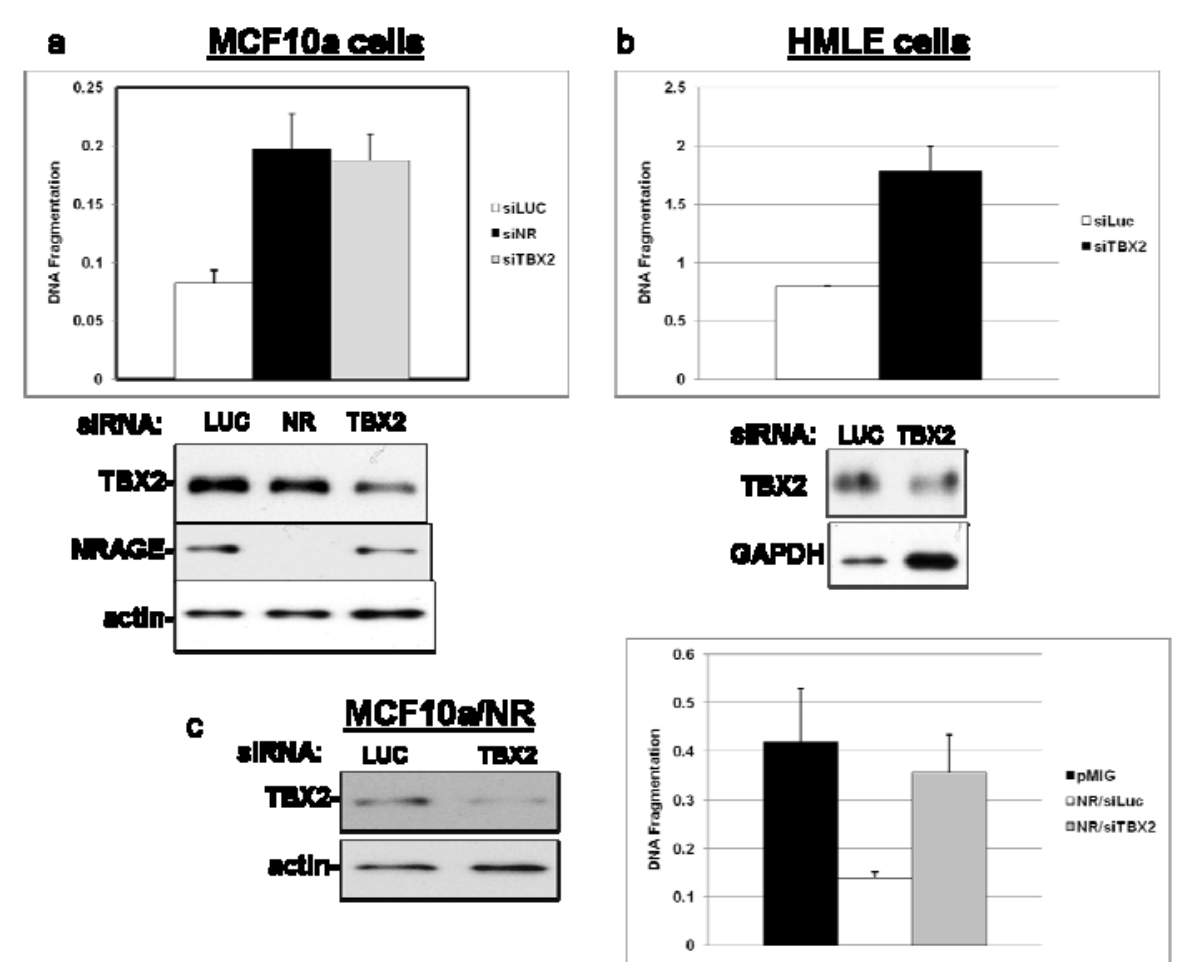

Fig.17. TBX2 and NRAGE interactions are functionally significant. TBX2 is an anoikis suppressor. (a) MCF10a cells or (b) HMLE cells were transfected with siRNA duplexes specific for luciferase ("siluc"), NRAGE ("siNR") or TBX2 ("siTBX2") and assayed for anoikis by DNA fragmentation ( $y$-axis represents optical density units). Both cell lines were assayed for anoikis at about 42 hours post-transfection; suspension time: 6 hours (MCF10a); 8 hours (HMLE). The knockdown was confirmed by immunoblot analysis. (c) TBX2 knockdown restores anoikis sensitivity in MCF10a+NRAGE cells. MCF10a+NRAGE cells were transfected with siRNA for luciferase ("siLuc") or TBX2 ("siTBX2") and assayed for anoikis by DNA fragmentation along with MCF10a+pMIG cells. The knockdown was confirmed by immunoblots. 
With NRAGE interaction, we were curious to see whether TBX2 regulates anoikis as well. The transient knockdown of TBX2 enhanced anoikis in MCF10a (fig.17a) and HMLE cells (fig.17b), suggesting its role similar to NRAGE as anoikis suppressor.We tested the effect of TBX2 on MCF10a + NRAGE; a partially anoikis resistant cells. The transient knockdown of TBX2 restored anoikis sensitivity in NRAGE-expressing cells (fig.17c). The data suggested that NRAGE effect on anoikis resistance required TBX2, confirming their co-operation for suppressing anoikis.

SW13 adrenocortical carcinoma cells have previously been shown to become anchorage-independent in presence of TBX2 over-expression (Ismail and Bateman, 2009). We tested whether NRAGE was required for promoting TBX2-dependent anchorage independent growth. SW13 cells were stably selected for TBX2 expression, followed by stable downregulation of NRAGE using retroviral shRNA. The anchorage independent growth was compared with control cells expressing pRS vector control. The knockdown of NRAGE suppressed anchorage-independent growth without affecting normal growth under attached conditions (fig.18). These data confirm the co-operation between NRAGE and TBX2 in promoting anchorage independent growth.

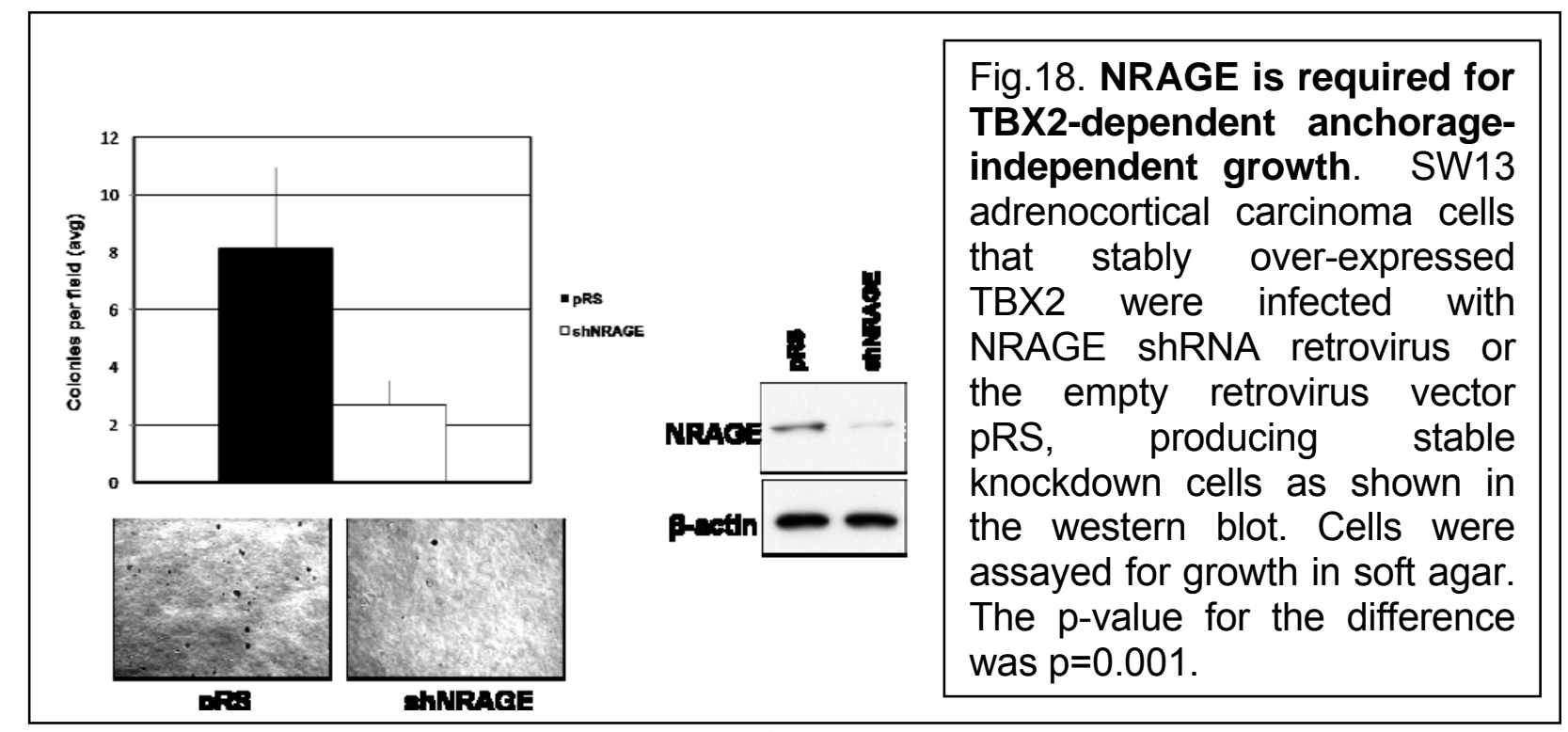


TBX2 has also been shown to promote anoikis resistance in EMT cells (HMLE+shECAD and HMLE+Twist cells) (Frisch SM, unpublished). The overall data suggest that the NRAGE and TBX2 co-operate for anoikis resistance and anchorage independent growth and so, hypothesized to promote tumor progression and metastatic growth.

Ankyrin-G regulates NRAGE nucleocytoplasmic localization: NRAGE interacts with proteins such as transcription factors, membrane receptors or proteins with cytoplasmic function and so can localize to nucleus, membrane or cytoplasm. In neuronal PC12 cells, NRAGE localized to membrane in response to NGF treatment (Salehi et al., 2000). In another context, co-expression of NRAGE and homeodomain protein Msx2 promoted NRAGE nuclear localization (Matsuda et al., 2003). The localization patterns of NRAGE in epithelial and EMT cells are not well characterized. We hypothesized that interacting partners such as ankyrin-G might regulate the nucleocytoplasmic transport of NRAGE.

To test the hypothesis that ankyrin-G might regulate NRAGE nucleocytoplasmic transport, we used the fibrosarcoma cell line HT1080, expresses very low level of endogenous ankyrin-G (unpublished observation). The cells were transfected either with HA-NRAGE and vector control, ankyrin-G or dominant negative ankyrin-G lacking UPA domain ( $\triangle \mathrm{UPA}$ ). NRAGE on its own localized to nucleus whereas co-expression of ankyrin-G sequestered NRAGE in cytoplasm. Expression of ankyrin-G lacking UPA failed to sequester NRAGE in nucleus (fig.19a). The data suggested that ankyrin-G sequesters NRAGE in cytoplasm which otherwise localizes to nucleus. Transient overexpression of NRAGE and ankyrin-G is an artificial system, so we tested the effect on ankyrin-G on NRAGE localization in more physiological context. 

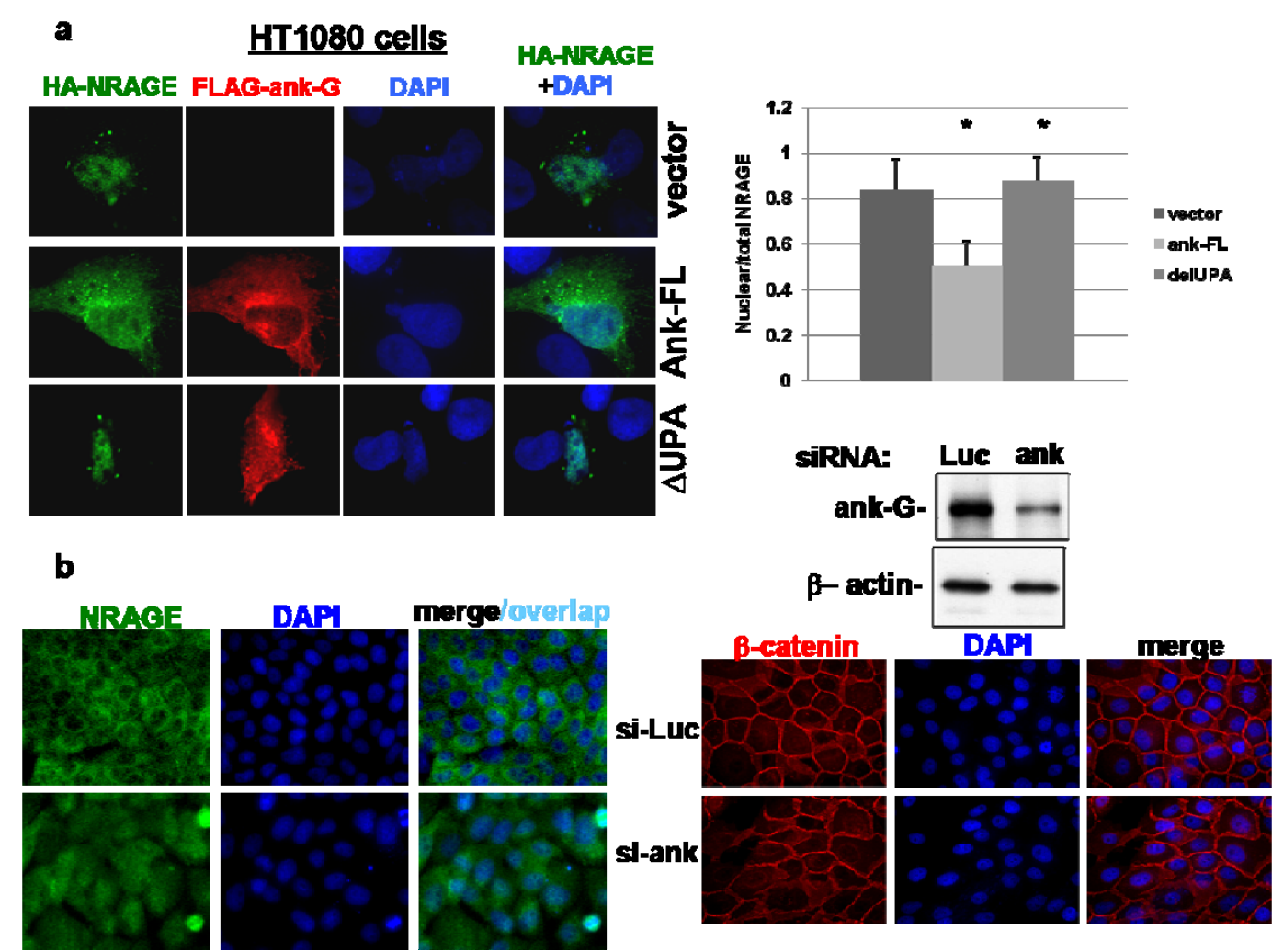

Fig.19. Ankyrin-G controls NRAGE localization. (a) Over-expressed ankyrin-G sequesters NRAGE in the cytoplasm. HT1080 cells were cotransfected with expression constructs encoding FLAG-tagged human ankyrin-G (210 kDa isoform), an NRAGE-non-binding ankyrin-G deletion mutant, $\triangle U P A$, or empty vector together with HA-NRAGE. Cells were then fixed and stained for FLAG-ankyrin-G, HA-NRAGE or chromatin (DAPI). The histogram represents the fraction of HA-NRAGE that was nuclear; $N=30, p=0.001$. (b) Depletion of ankyrin- $G$ facilitates the accumulation of NRAGE in the nucleus. MCF10a cells were transfected with siRNA for ankyrin-G ("siAnk") or luciferase ("si-Luc"). Cells were then treated with leptomycin B for 30 minutes prior to fixation and stained for NRAGE (green, left panel) or chromatin (DAPI, blue) or B-catenin (red, right panel)). For the merged images, color intensities were adjusted to provide optimal color balance. ( $\mathrm{N} \geq 2)$

We used the MCF10a cells and knocked down ankyrin-G through transient transfection. Before fixing cells, leptomycin B (inhibitor of Crm1 dependent nuclear export) was treated for 30 minutes. Transient knockdown of ankyrin-G accumulated NRAGE protein in nucleus compared to luciferase siRNA-treated control cells (fig.19b). As a control, beta catenin staining for luciferase and ankyrin-G knocked down cells was 
performed and no noticeable difference was observed. To rule out the NRAGE antibody artifact, we performed similar experiment in MCF10a+NRAGE cells and HA staining was performed. As observed previously, transient knockdown of ankyrin-G accumulated exogenous NRAGE in nucleus (fig.20). These results indicated that ankyrin-G regulated the nucleocytoplasmic localization of NRAGE by direct interaction.

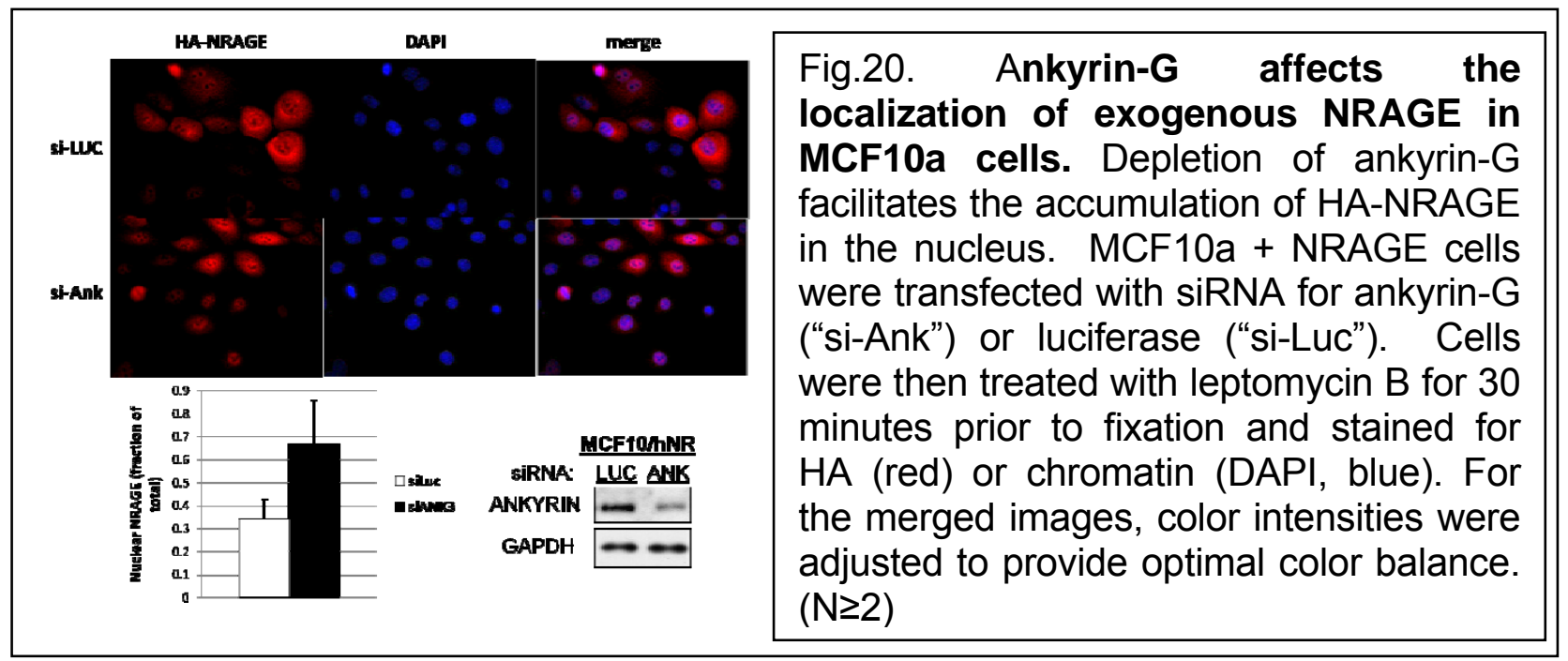

EMT promotes nuclear localization of NRAGE: Induction of EMT either by stable knockdown of E-cadherin or Twist overexpression in HMLE suppressed ankyrin-G expression when compared with parental HMLE cells ( 210 kDa, fig. 1C, chapter 3). We tested if these cells have different NRAGE localization pattern in comparison to parental HMLE cells and whether they correlate with the observations made in MCF10a-/+ankyrinG cells (fig.19 and 20). The NRAGE localization data suggested that the cells undergone EMT (HMLE+shECAD and HMLE+Twist) accumulate NRAGE in the nucleus significantly compared to the control HMLE cells (fig.21a). We also made an interesting observation that NRAGE protein fused to multiple SV40 T-antigen nuclear localization signals (NLS) were selected stably in HMLE cells, displayed a cytoplasmic localization pattern 
compared to predominantly nuclear localization in HMLE+Twist cells. These observations suggest that the epithelial cells show strong selection for cytoplasmic NRAGE; probably ankyrin-G sequesters NRAGE in cytoplasm (fig.21b). These observations indicate that the ankyrin-G downregulation in the cells which have undergone EMT as well as in the variety of human tumors, promote the nuclear localization of NRAGE.
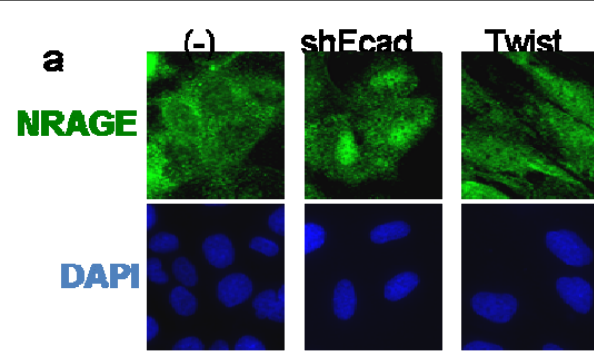

b
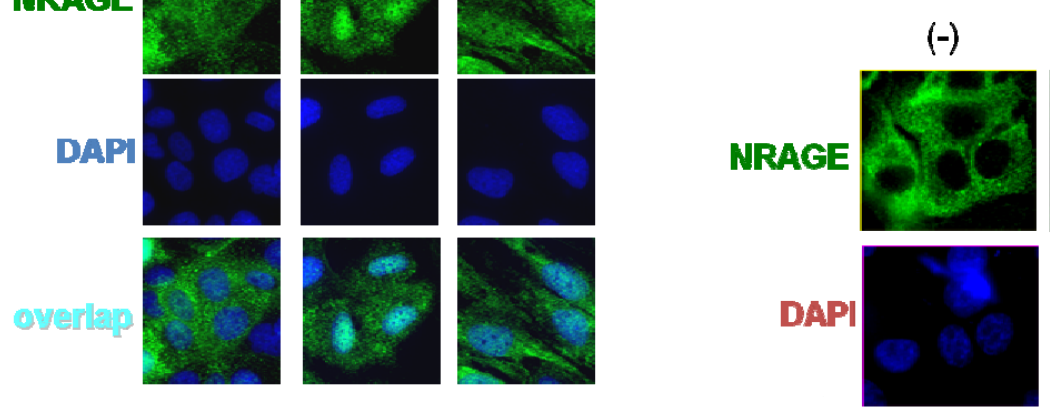

Twist
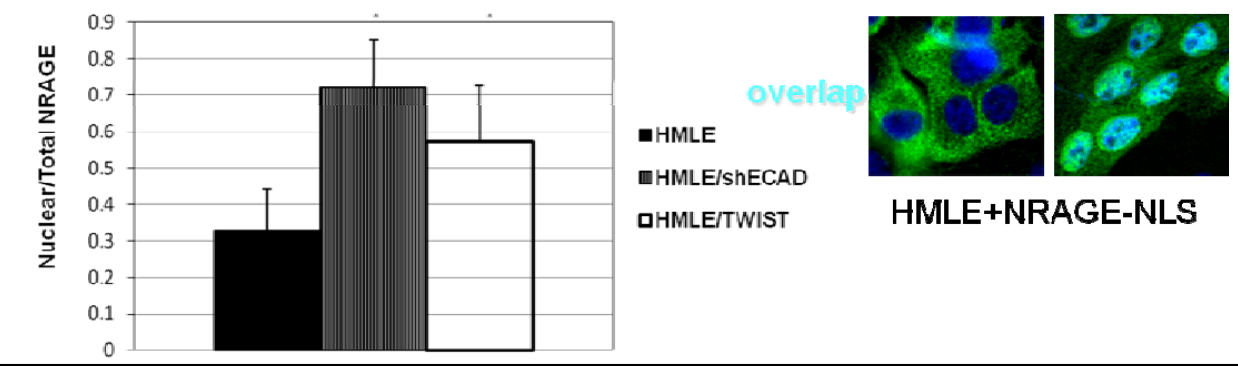

HMLE+NRAGE-NLS
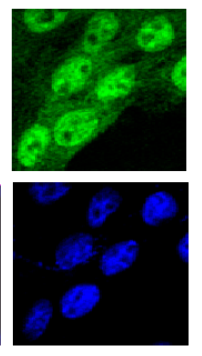

HIMLE+NRAGE-NLS

Fig.21. EMT promotes nuclear accumulation of NRAGE. (a) The HMLE, HMLE+shEcad or HMLE+Twist cells were treated with leptomycin $B$ for thirty minutes prior to fixation and staining for NRAGE (green) or chromatin (DAPI, blue); $p=0.001$. $(\mathrm{N} \geq 3)$ (b) NRAGE-NLS accumulates in the nucleus of cells over-expressing Twist. Normal HMLE cells or HMLE+Twist cells were stably transduced with an HA-NRAGENLS-expressing retrovirus. Cells were fixed and stained for NRAGE (green) and chromatin (DAPI, blue) without leptomycin B treatment. For the merged images, color intensities were adjusted to provide optimal color balance. ( $\mathrm{N} \geq 2)$

To test if NLS-NRAGE localization in HMLE cells were mainly due to the presence of ankyrin-G, we transiently knocked down ankyrin-G from HMLE + NLS-NRAGE cells. The loss of ankyrin-G showed significantly higher nuclear NRAGE compared to luciferase 
siRNA transfected control cells (fig.22). Considering that the NLS-NRAGE is overexpressed and all the cells were not positive for siRNA transfection, relocalization data confirmed our hypothesis and previous observations that ankyrin-G regulated NRAGE nucleocytoplasmic transport.

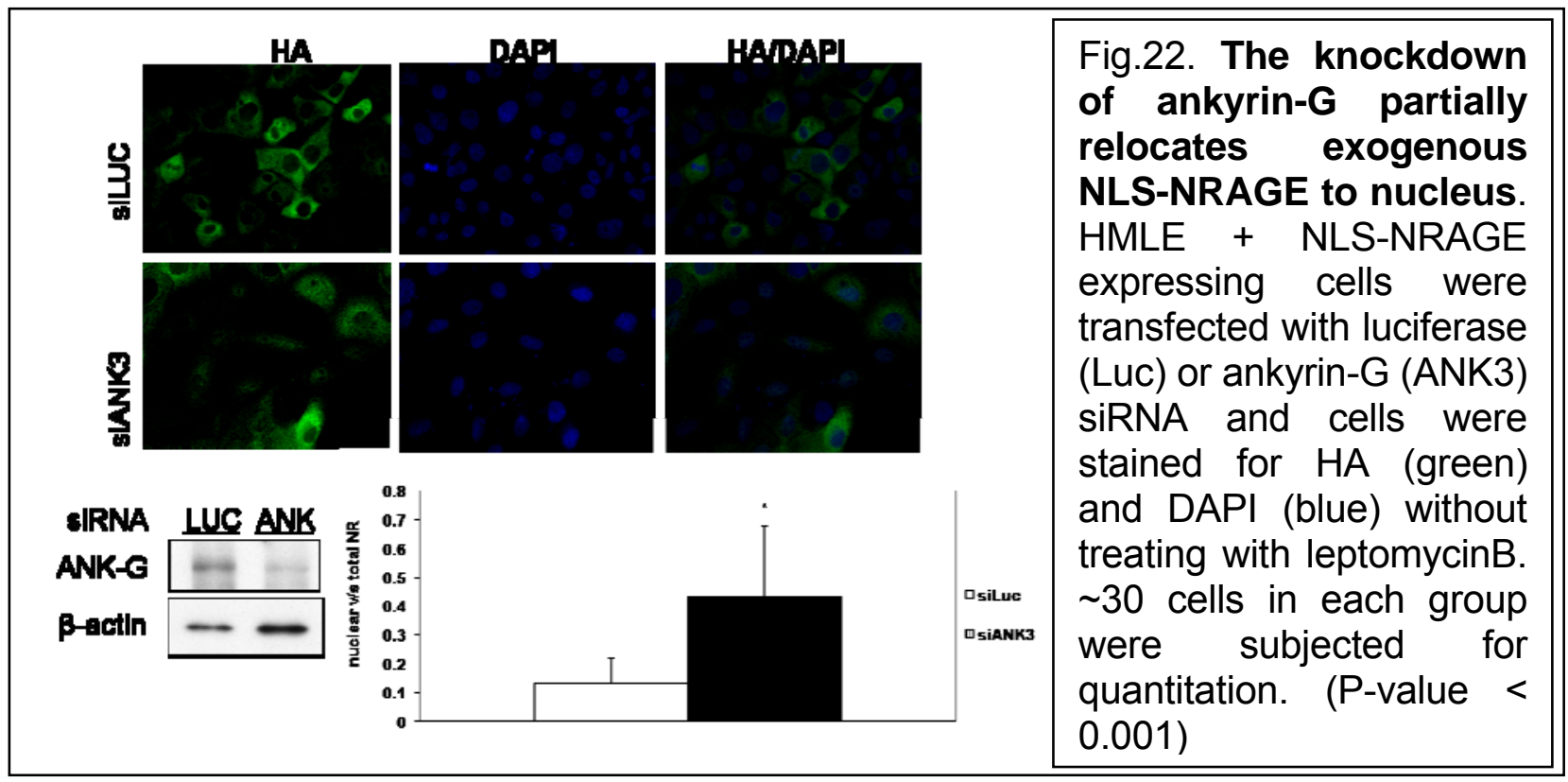

Ankyrin counteracts NRAGE-mediated transcription repressor function: NRAGE interacts with transcription factors such as DLX5, Msx2, and Tbx2 etc. Our data indicated that NRAGE sub-cellular localization depends on the level of ankyrin-G. NRAGE has previously been reported to regulate Dlx5-dependent transcription activity using luciferase assay based mammalian 2-hybrid assay (Masuda et al., 2001; Sasaki et al., 2002). We used similar strategy and used the c-terminal deletion construct of DIx5 fused to Gal4 DNA binding domain (pBind-Dlx5 $\Delta \mathrm{C}$ ) and transfected with Gal4 activation domain containing construct (pG5-luc) fused with luciferase gene. Co-transfection of pBindDlx5 $\Delta \mathrm{C}$ and pG5-luc activated transcription compared to pBind vector control. We then 
tested the effect of NRAGE knockdown in DIx5 dependent transcription activity. NRAGE knockdown enhanced Dlx5 mediated transcriptional activity, suggesting its role as transcription repressor (fig.23). We further tested the role of ankyrin DIx5 transcriptional activity. The over-expression of ankyrin-G enhanced DIx5 dependent luciferase activity, whereas transient knockdown of ankyrin-G repressed Dlx5 mediated transcription activity (Fig.24). The effect of ankyrin-G expression on Dlx5 transcription activity matched with our hypothesis based on NRAGE-mediated transcriptional repression and ankyrin effect on NRAGE localization. This is the first ever observation that a cytoskeletal protein ankyrin-G regulates transcription, possibly by counteracting NRAGE dependent transcriptional repression. The physiological relevance of ankyrin-G/NRAGE regulation of Dlx5 mediated transcription activity is presently unknown.

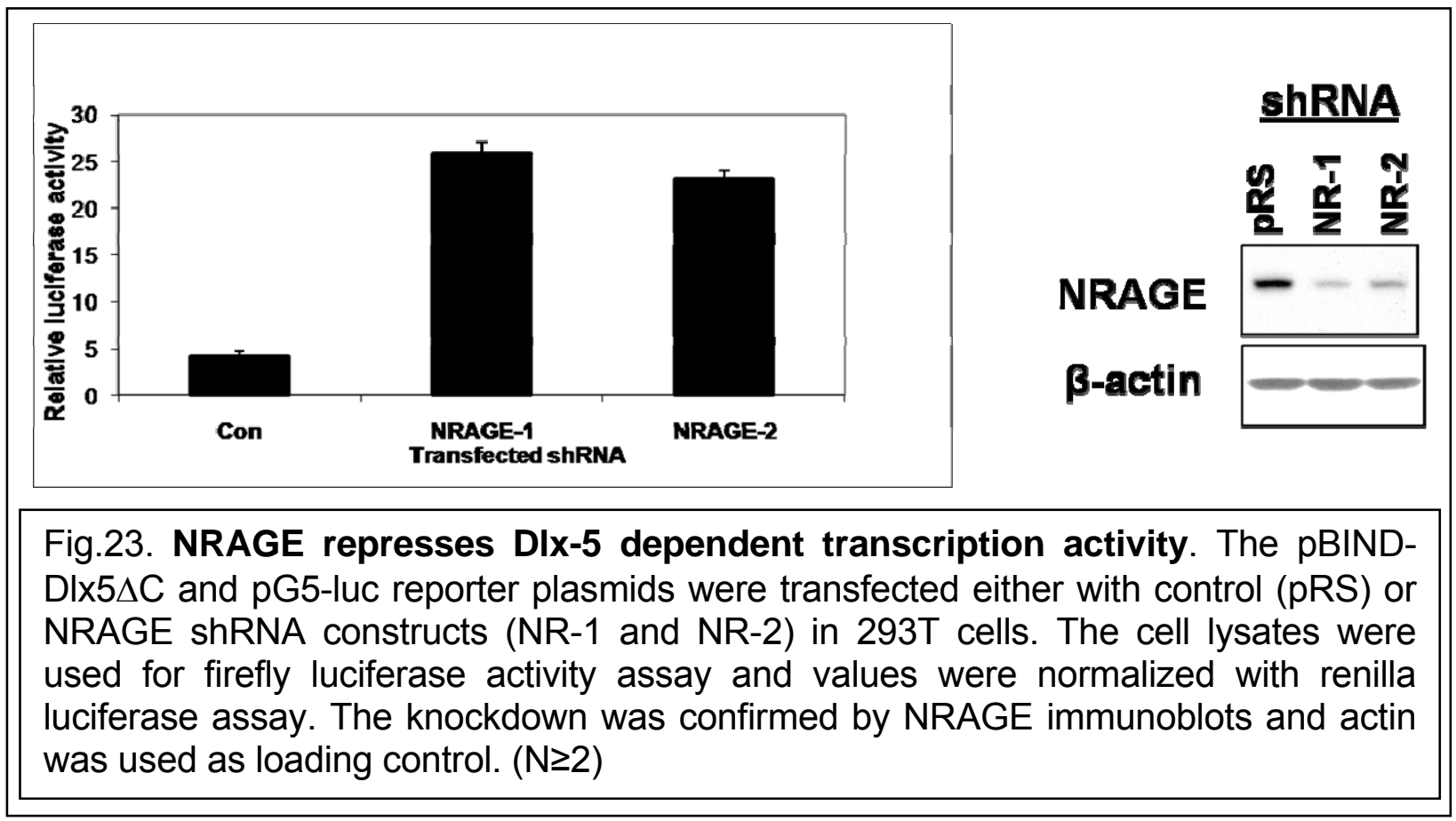




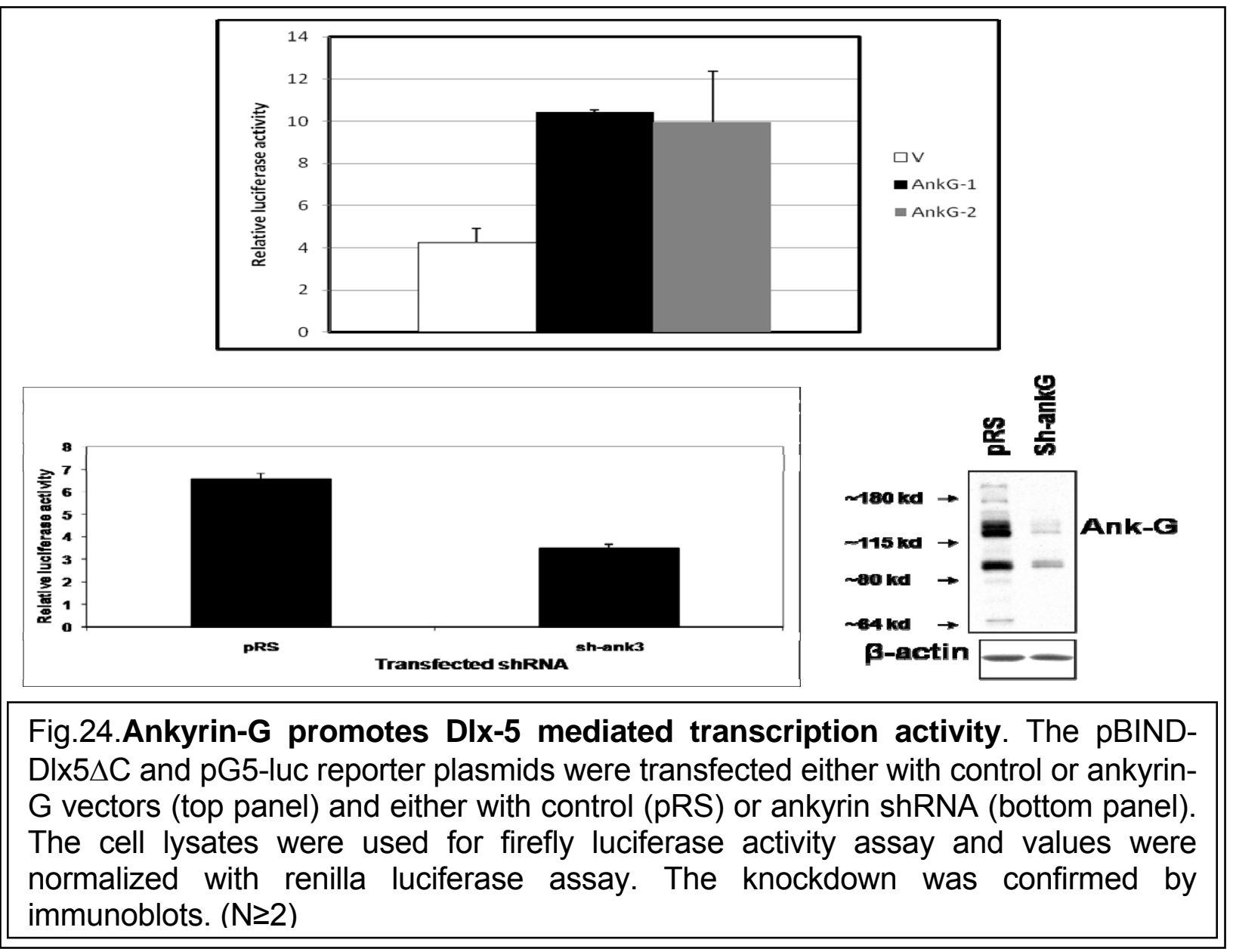

The novel NRAGE-interacting partner TBX2 is a well known transcriptional repressor and its best characterized target is tumor suppressor p14-ARF (Jacobs et al., 2000; Prince et al., 2004; Rodriguez et al., 2008). Experiments performed in our laboratory using luciferase based transcriptional assay and chromatin immunoprecipitation (CHIP) suggested that NRAGE acted as a co-repressor for TBX2 mediated p14-ARF repression through binding to T-Box element. NRAGE and TBX2 suppress anoikis and repress p14-ARF expression. Do NRAGE and TBX2 anoikis suppression depend on ARF? Is ARF an anoikis promoter? We decided to test p14ARF/p19-ARF for its role in anoikis. 
ARF regulates anoikis: NRAGE and TBX2 co-operate in suppressing anoikis and promoting anchorage independent growth. TBX2 target p14-ARF has been shown to have pro-apoptotic activities. We hypothesized that p14-ARF promoted anoikis. To test the effect of p14-ARF (p19-ARF in mouse) on anoikis, we compared p19ARF-knockout mouse embryo fibroblasts (MEFs) or matched wild-type control MEFs, immortalized with SV40 T-antigen cells for anoikis. The p19ARF-knockout MEFs were dramatically less sensitive to anoikis compared to the wild-type MEFs (fig.25), confirming that p14ARF/p19-ARF promoted anoikis, indicating a novel function for this tumor suppressor gene.

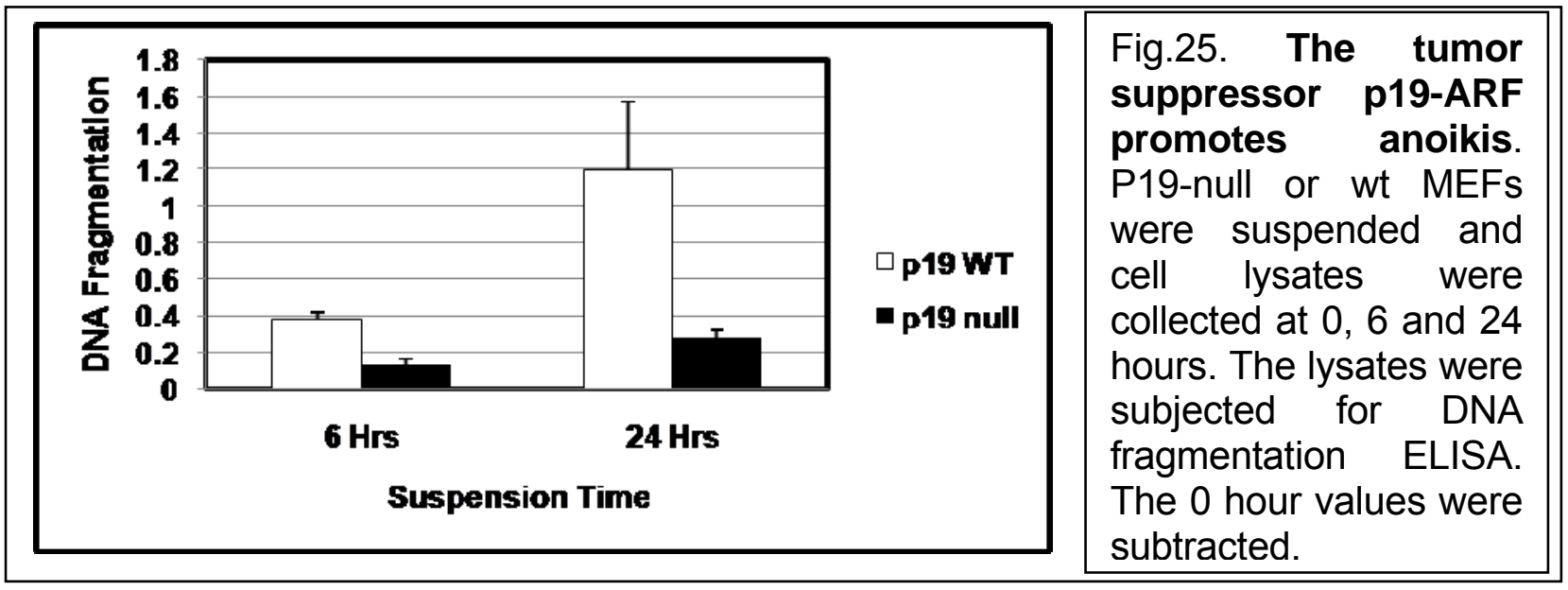

We also compared two MCF10a cell variants, one obtained from ATCC which lack both alleles of ARF and the other obtained from Karmanos Cancer Center (K) which contains one functional allele of ARF locus (Frisch SM, unpublished). We transiently knocked down NRAGE in both the MCF10a variants (ATCC and K) and compared the cell lysates for anoikis. The MCF10a cells lacking both ARF alleles (ATCC) found to be completely resistant to anoikis and NRAGE didn't affect anoikis sensitivity either (fig.26). 


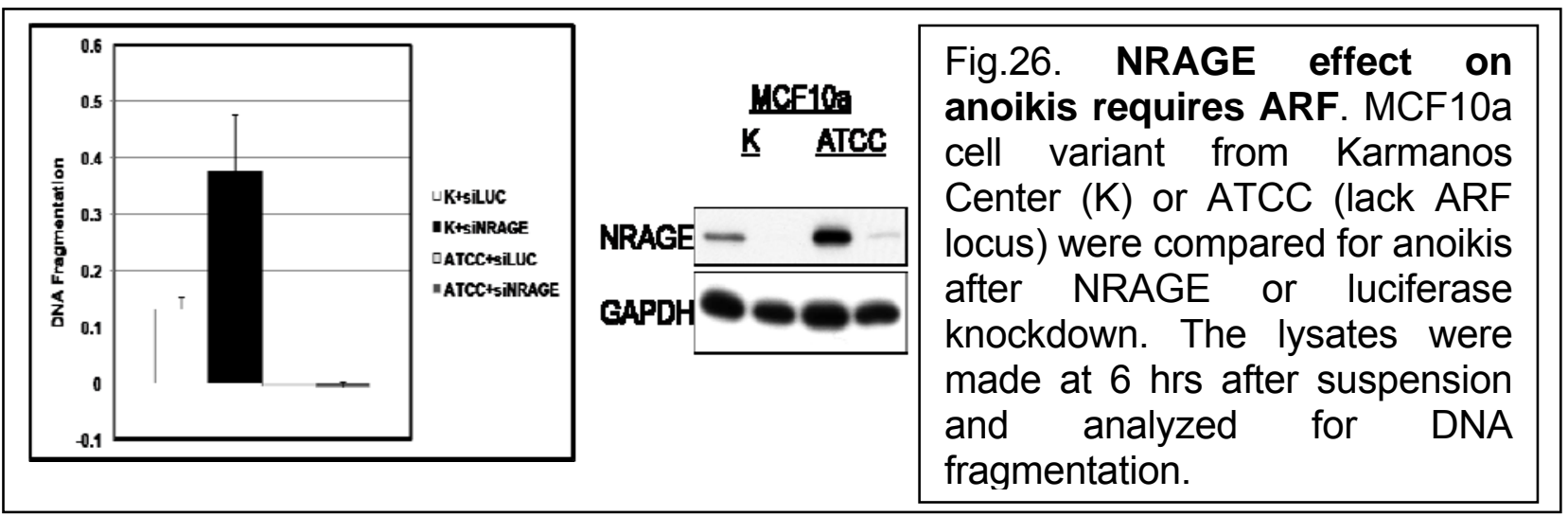

Expression of nuclear NRAGE suppresses anoikis: NRAGE localization is controlled by ankyrin-G in epithelial cells. The stable over-expression of NRAGE shows cytoplasmic localization. We wanted to know if nuclear NRAGE could suppress anoikis. The stably expressed NRAGE with multiple SV40 T-antigen nuclear localization signals (NLS) showed predominantly cytoplasmic localization in epithelial cells (MCF10a and HMLE). Individual clones showing cytoplasmic or nuclear localization were selected and compared for anoikis sensitivity. The first set of cells compared for anoikis were HMLE + NLS-NRAGE1 (with cytoplasmic localization) and HMLE + NLS-hNR3 (nuclear localization) (fig.27a). Surprisingly, both of these HMLE clones suppressed anoikis significantly (fig.27b). Another HMLE clone, NLS-hNR25 expressing nuclear localization was compared for anoikis with control cells (pMIG) and it suppressed anoikis equally well as NRAGE with coding sequence (CDS-hNR) (fig.27c.). Similarly, MCF10a cells with nuclear NRAGE (NLS1 and NLS8) were compared for anoikis, behaved similar to HMLE cells and suppressed anoikis compared to control cells (fig.27d.). This data suggested that NRAGE localized in nucleus suppressed anoikis. The cytoplasmic expressing NRAGE (HMLE + NLS-NR1) also suppressed anoikis. To test the effect of cytoplasmic 
NRAGE, NES-NRAGE (containing nuclear exclusion signal) expressing HMLE and MCF10a cells were generated and compared for anoikis.

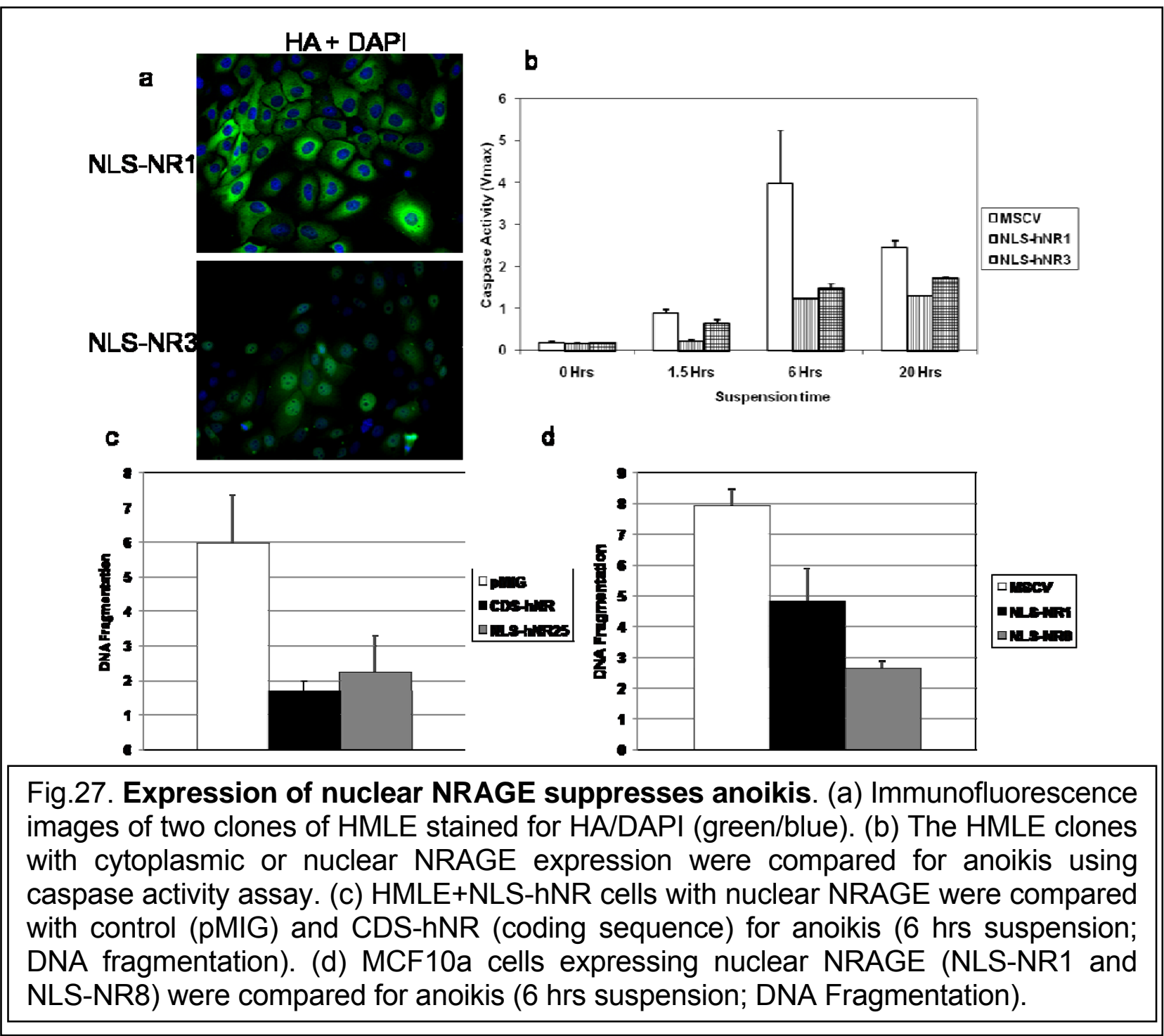

Cytoplasmic NRAGE suppresses anoikis: The HMLE and MCF10a cells were selected for NRAGE containing nuclear exclusion signal (NES-NRAGE). Selection of cytoplasmic expressing NRAGE (NES-NRAGE) clones was far easier compared to nuclear expressing NRAGE as majority of cells showed transgene expression. The 
anoikis data suggested that cytoplasmic NRAGE (NES) suppressed anoikis equally well as CDS-NRAGE (with coding sequence) or NLS-NRAGE in subconfluent and confluent MCF10a and HMLE cells (fig.28). We have observed previously that loss of ankyrin-G during EMT promoted nuclear localization of NRAGE (fig.21-24). In presence of ankyrinG, NRAGE localized to cytoplasm and its overexpression affected cell polarity and adherens junction kinetics (fig.8 and fig.9).

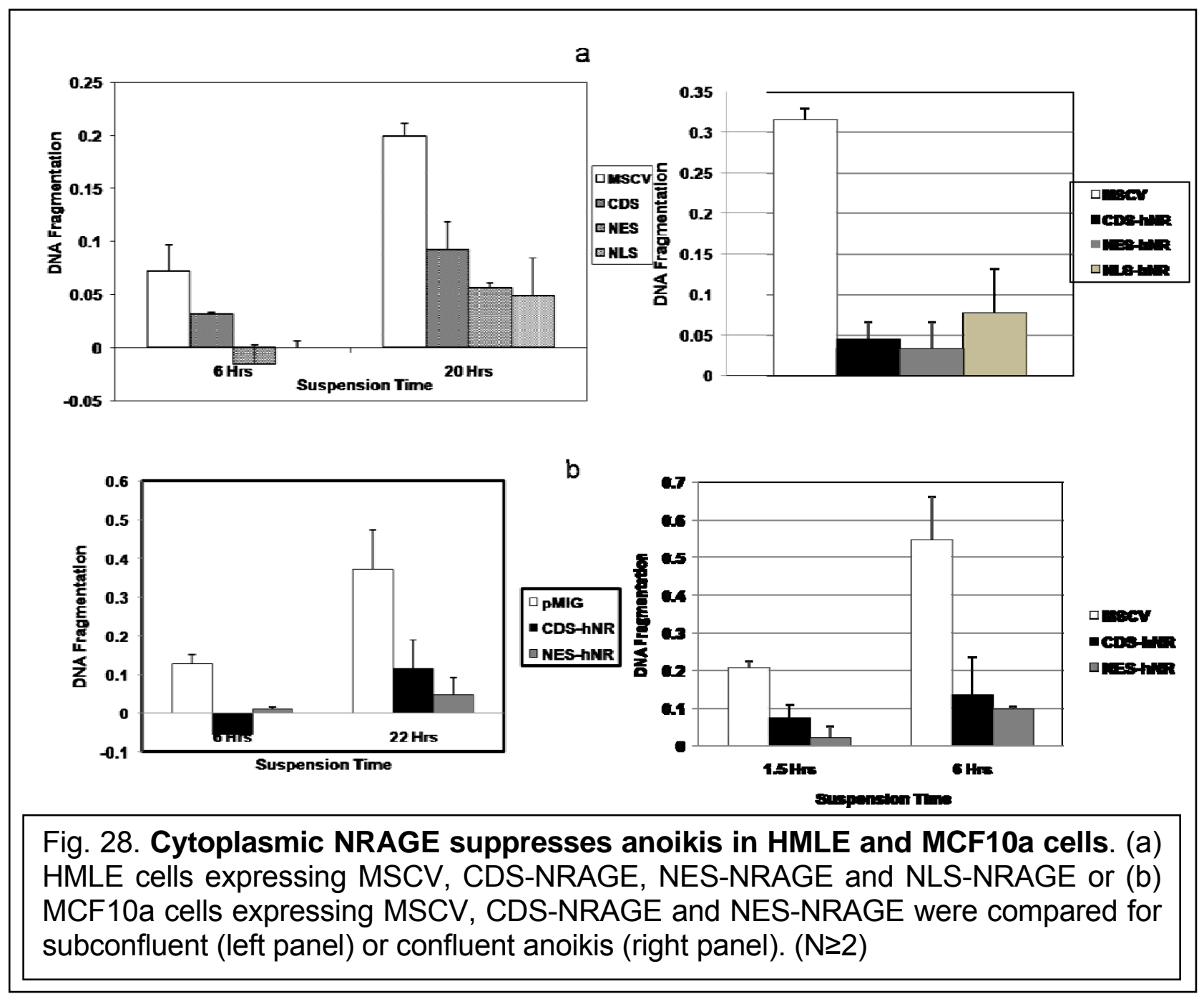


NRAGE promotes survival signaling in cells lacking cell-extracellular matrix interaction: The expression of cytoplasmic NRAGE (NES-NRAGE) suppressed anoikis in HMLE and MCF10a cells. We hypothesized that NRAGE might regulate signaling process, an effect of misregulated adherens junction function. We first tested the effect of NRAGE knockdown on AKT and p38 activation in early detached MCF10a cells. The immunoblot data suggested that NRAGE retained AKT phosphorylation in early detached cells (fig.29). It is previously reported that retention of activated AKT suppress anoikis and promote survival in suspended cells (Frisch and Ruoslahti, 1997). We made similar

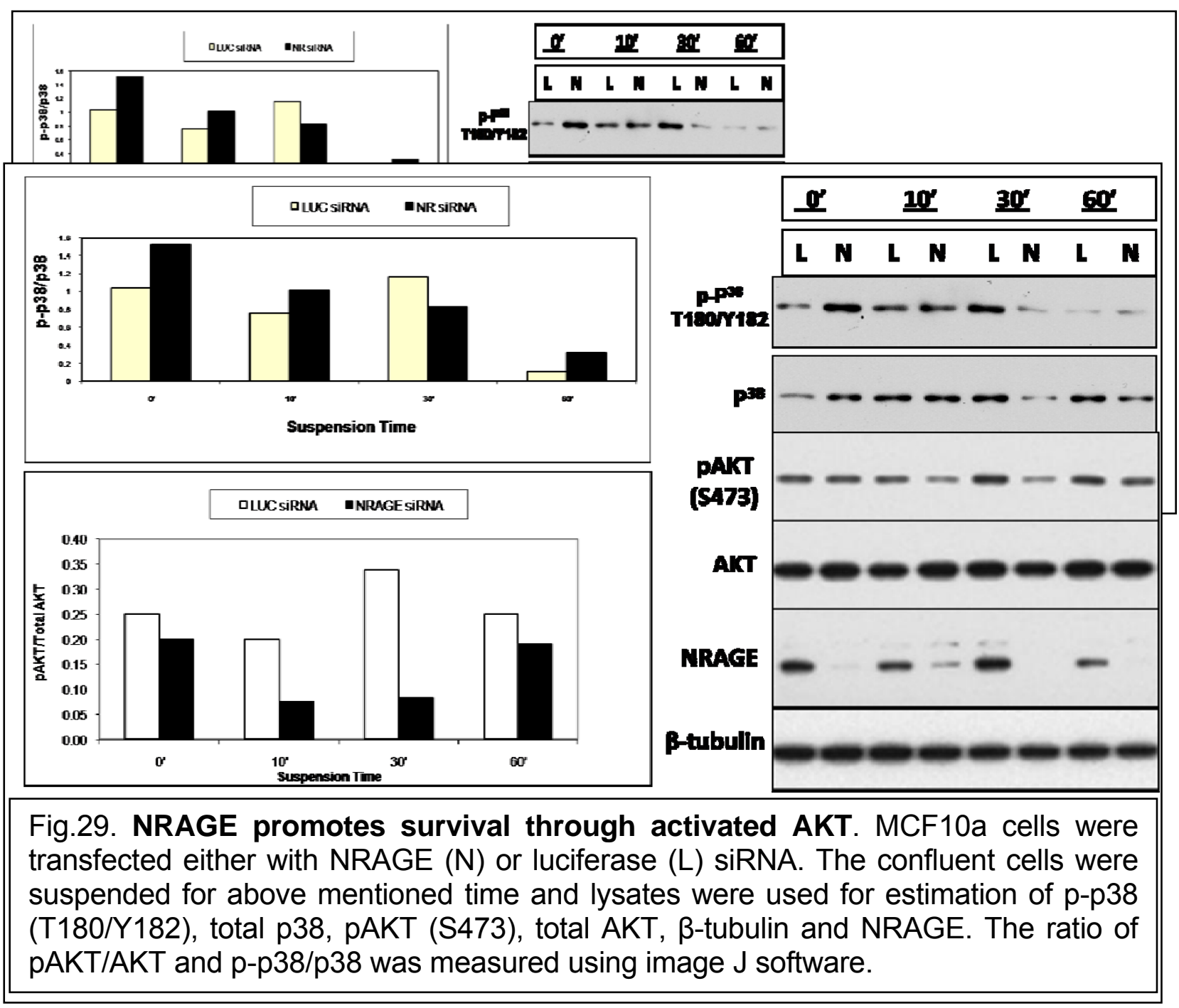


Based on NRAGE effect on AKT activation during short term suspension, we decided to test the signaling events using low density culture in long term suspension. The MCF10a (-/+ NRAGE) cells were suspended and lysates were collected at various time points. The immunoblots were compared for various signaling molecules such as phospho-AKT, phospho-FAK, phospho-ERK and phospho-EGFR. The data suggested that the NRAGE over-expression affected the AKT and FAK activity whereas effect on EGFR and ERK was very minimal in long time suspension (fig. 30a).

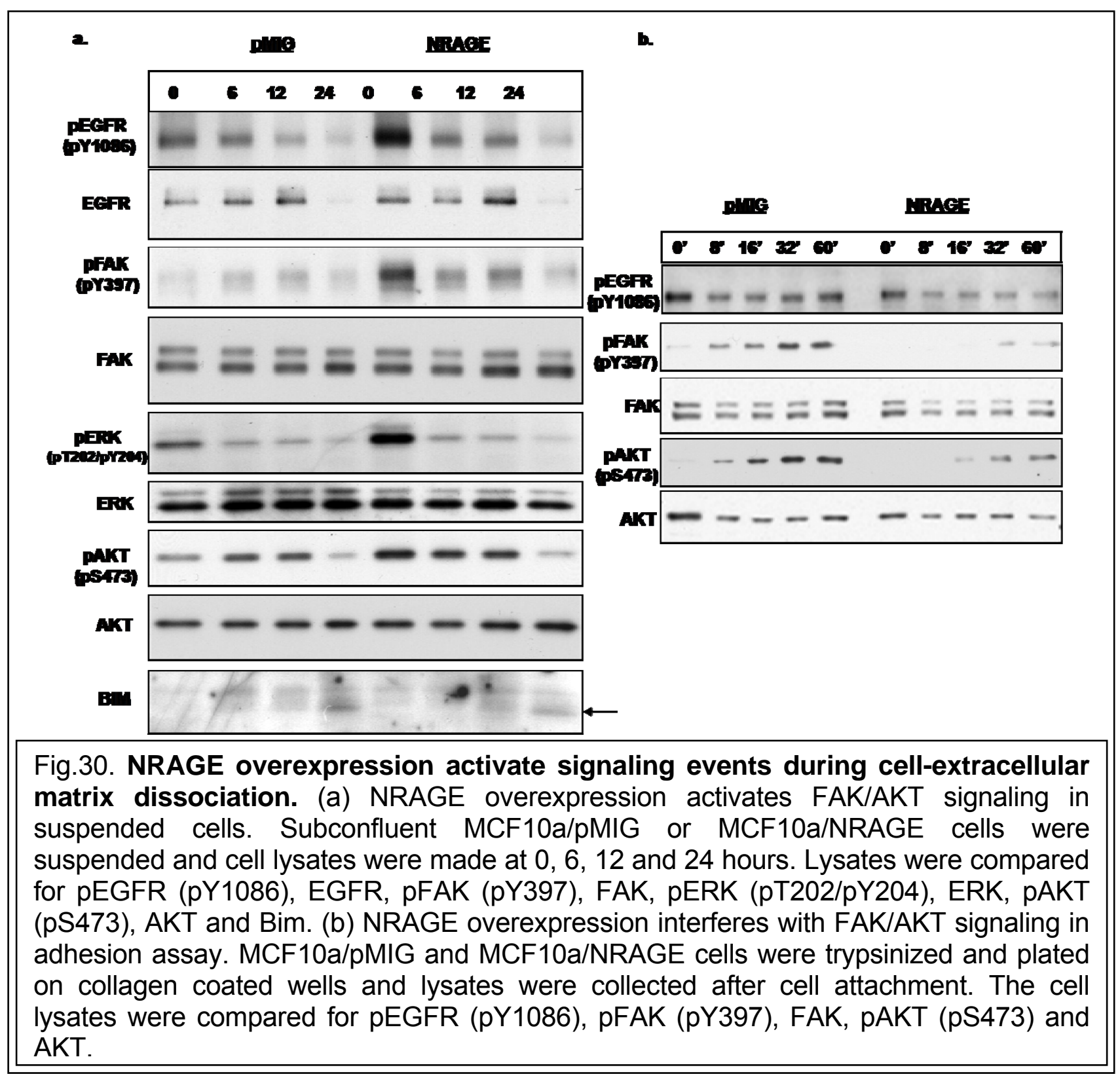


We further compared the effect of NRAGE overexpression in collagen re-adhesion assay where MCF10a (-/+NRAGE) cells were plated on collagen and collected lysates were immunoblotted for pFAK/FAK, pAKT/AKT, and EGFR. Surprisingly, NRAGE expressing cells were dramatically deficient in AKT and FAK activation in cells attached on collagen (fig.30b). The data indicate that NRAGE promotes survival signaling through FAK and AKT in suspended cells and interferes with early signaling events in cells reattached on collagen. The overall data suggest that NRAGE overexpression affected prosurvival signaling events during suspension and inhibited signaling events during early adhesion. It is yet to be verified if the signaling events during suspension correlate with the cell adhesion, polarity and adherens junction kinetics. The cells dissociated from extracellular matrix with better pro-survival signaling could promote cell metastasis. Discussion

The MAGE family protein, NRAGE/MAGE-D1 was identified as Unc5H1 interaction partner in a yeast 2-hybrid screen using E18 murine brain tissue cDNA library. The MAGE homology domain and C-terminal domain of NRAGE interacted with ZU5 domain and adjacent sequence (Williams et al., 2003). These unc5H1 regions were later characterized as ZU5-UPA-DD supermodule, homologous in Unc5, PIDD and ankyrins (Wang et al., 2009). Based on structural homology, NRAGE was tested for interaction with ankyrin-G. The interaction was confirmed in HMLE and MCF10a cells. The UPA (homolgous in Unc5, PIDD and ankyrin) domain of ankyrin-G was identified as domain responsible for interaction with MAGE homology domain and C-terminal domain of NRAGE (fig.1, 2, 3, and 5). The ZU5-A domain (part of spectrin binding domain) and Cinsert (in C-terminal regulatory domain) of ankyrin-G interfered with NRAGE interaction 
(fig. 4, 6, and 7). The ZU5-A interference to NRAGE interaction might be due to closed structural conformation. Our data suggest that ZU5-A domain alone could interact with NRAGE (unpublished observation). In invitro interaction studies, NRAGE competed with spectrin for ankyrin-G binding (Frisch SM, unpublished observation). Overexpression of NRAGE affected localization and detergent solubility of ankyrin-G (fig.8). NRAGE also suppressed ankyrin-G induced apoptosis, affected E-cadherin localization and adherens junction kinetics (fig. 8 and 9). NRAGE has previously been implicated in disrupting Ecadherin/beta catenin complex and so might affect the adherens junction and cell polarity (Xue et al., 2005).

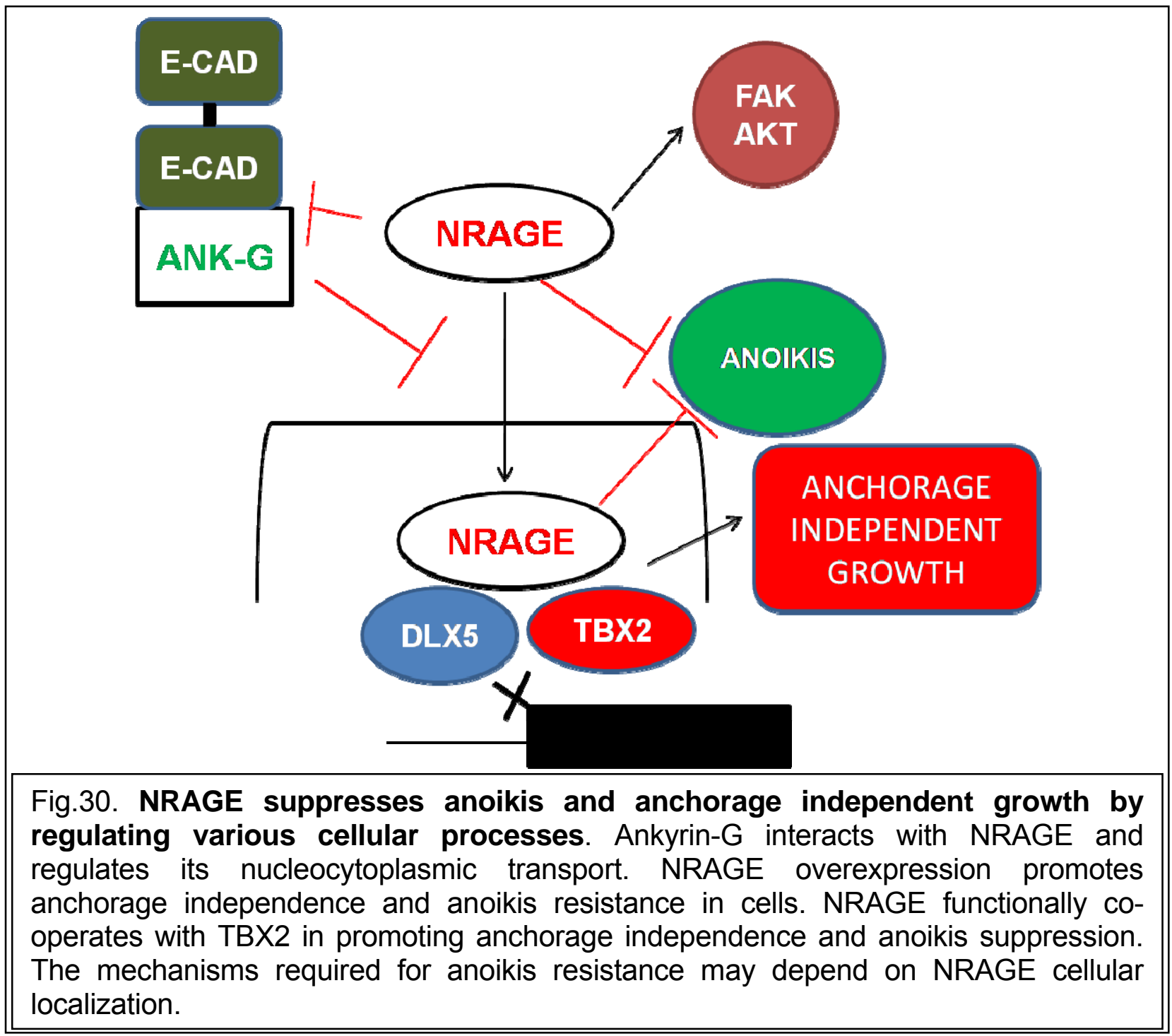


Loss of cell-extracellular matrix interaction promotes cytoplasmic localization (from cytoskeleton) of ankyrin-G with elevated protein level (chapter 3; fig.2 and fig.3). To mimic this condition invitro, we overexpressed ankyrin-G. NRAGE suppressed ankyrin-G dependent apoptosis. Based on this observation, we went ahead and tested the effect of NRAGE on anoikis. By transient knockdown and stable overexpression, we established NRAGE as an anoikis suppressor. The NRAGE role as anoikis suppressor correlated well with its expression in breast cancer cells and EMT. Our data also support previous observations of NRAGE upregulation in lung, kidney and head/neck carcinoma (Bhattacharjee et al., 2001; Boer et al., 2001; Ginos et al., 2004).

We performed yeast 2-hybrid screening and identified oncogenic transcription repressor TBX2, as NRAGE interacting partner. TBX2 and NRAGE co-operated for suppressing anoikis sensitivity and promoting anchorage independent growth. NRAGE is predicted to be TBX2 co-repressor and its role is yet to be verified in physiological context. TBX2 is known to repress p14-ARF/NDRG1 and NRAGE function as a corepressor is yet to be verified (Jacobs et al., 2000; Prince et al., 2004; Redmond et al.).

NRAGE was identified as lateral membrane protein ankyrin-G interaction partner. We hypothesized that ankyrin-G might regulate NRAGE nucleocytoplasmic transport. Ankyrin-G expression sequestered NRAGE in cytoplasm whereas dominant negative expression construct lacking UPA domain (ankyrin-G $\triangle$ UPA) failed to do so, indicating that the direct interaction was responsible for NRAGE cellular localization. Based on ankyrin$G$ effect on NRAGE localization, we tested the effect of ankyrin on DIx5 transcription activity. Ankyrin-G counteracted NRAGE mediated regulation on DIx5 transcriptional 
activity (fig.23 and 24). Here we show a novel function of cytoskeletal protein ankyrin-G in regulating nucleocytoplasmic transport and transcriptional activity.

We further tested if cytoplasmic or nuclear forms of NRAGE suppress anoikis. Surprisingly, both forms suppressed anoikis. The nuclear form of NRAGE affects anoikis by co-operating with TBX2. The mechanism of cytoplasmic NRAGE in anoikis is not clear. NRAGE affected ankyrin-G sub-cellular localization and adherens junction kinetics, hence we hypothesized that NRAGE might promote pro-survival signaling process. In the experimental set up, NRAGE promoted pro-survival signaling in cells dissociated from extra-cellular matrix and inhibited early signaling events in collagen adhesion assay (fig.30).

Our data suggested that ankyrin-G interacting MAGE family protein NRAGE suppressed anoikis. Ankyrin-G sequesters NRAGE in cytoplasm. During EMT, ankyrin-G is downregulated, which in turn allows NRAGE to localize to nucleus. NRAGE cooperates with TBX2 in suppressing anoikis and promoting anchorage independent growth. Cytoplasmic NRAGE also suppressed anoikis, probably by affecting ankyrin-G mediated membrane function, adherens junction kinetics, and promoting pro-survival signaling in cells dissociated from extracellular matrix (fig.30). 


\section{CHAPTER 5}

\section{REFERENCES}

Abbi, S., and Guan, J.L. (2002). Focal adhesion kinase: protein interactions and cellular functions. Histology and histopathology 17, 1163-1171.

Abrahams, A., Parker, M.I., and Prince, S. The T-box transcription factor Tbx2: its role in development and possible implication in cancer. IUBMB life 62, 92-102.

Adams, J.M., and Cory, S. (1998). The Bcl-2 protein family: arbiters of cell survival. Science (New York, NY 281, 1322-1326.

Al-Hajj, M., Wicha, M.S., Benito-Hernandez, A., Morrison, S.J., and Clarke, M.F. (2003). Prospective identification of tumorigenic breast cancer cells. Proceedings of the National Academy of Sciences of the United States of America 100, 3983-3988.

Ango, F., di Cristo, G., Higashiyama, H., Bennett, V., Wu, P., and Huang, Z.J. (2004). Ankyrin-based subcellular gradient of neurofascin, an immunoglobulin family protein, directs GABAergic innervation at purkinje axon initial segment. Cell 119, 257-272.

Aoudjit, F., and Vuori, K. (2001). Matrix attachment regulates Fas-induced apoptosis in endothelial cells: a role for c-flip and implications for anoikis. The Journal of cell biology 152, 633-643.

Arumugam, T., Ramachandran, V., Fournier, K.F., Wang, H., Marquis, L., Abbruzzese, J.L., Gallick, G.E., Logsdon, C.D., McConkey, D.J., and Choi, W. (2009). Epithelial to mesenchymal transition contributes to drug resistance in pancreatic cancer. Cancer research 69, 5820-5828.

Ashkenazi, A., and Dixit, V.M. (1998). Death receptors: signaling and modulation. Science (New York, NY 281, 1305-1308.

Attwell, S., Roskelley, C., and Dedhar, S. (2000). The integrin-linked kinase (ILK) suppresses anoikis. Oncogene 19, 3811-3815.

Barker, P.A., and Salehi, A. (2002). The MAGE proteins: emerging roles in cell cycle progression, apoptosis, and neurogenetic disease. Journal of neuroscience research $67,705-712$.

Bates, R.C., Edwards, N.S., Burns, G.F., and Fisher, D.E. (2001). A CD44 survival pathway triggers chemoresistance via lyn kinase and phosphoinositide 3-kinase/Akt in colon carcinoma cells. Cancer research 61, 5275-5283. 
Bates, R.C., Elith, C.A., Thorne, R.F., and Burns, G.F. (1998). Engagement of variant CD44 confers resistance to anti-integrin antibody-mediated apoptosis in a colon carcinoma cell line. Cell adhesion and communication 6, 21-38.

Benoit, D.S., Tripodi, M.C., Blanchette, J.O., Langer, S.J., Leinwand, L.A., and Anseth, K.S. (2007). Integrin-linked kinase production prevents anoikis in human mesenchymal stem cells. Journal of biomedical materials research 81, 259-268.

Bertrand, M., Huijbers, I., Chomez, P., and De Backer, O. (2004). Comparative expression analysis of the MAGED genes during embryogenesis and brain development. Dev Dyn 230, 325-334.

Bertrand, M.J., Kenchappa, R.S., Andrieu, D., Leclercq-Smekens, M., Nguyen, H.N., Carter, B.D., Muscatelli, F., Barker, P.A., and De Backer, O. (2008). NRAGE, a p75NTR adaptor protein, is required for developmental apoptosis in vivo. Cell death and differentiation 15, 1921-1929.

Bhattacharjee, A., Richards, W.G., Staunton, J., Li, C., Monti, S., Vasa, P., Ladd, C., Beheshti, J., Bueno, R., Gillette, M., et al. (2001). Classification of human lung carcinomas by mRNA expression profiling reveals distinct adenocarcinoma subclasses. Proceedings of the National Academy of Sciences of the United States of America 98, 13790-13795.

Biswas, S.C., and Greene, L.A. (2002). Nerve growth factor (NGF) down-regulates the Bcl-2 homology $3(\mathrm{BH} 3)$ domain-only protein Bim and suppresses its proapoptotic activity by phosphorylation. The Journal of biological chemistry 277, 49511-49516.

Boatright, K.M., and Salvesen, G.S. (2003). Caspase activation. Biochemical Society symposium, 233-242.

Boer, J.M., Huber, W.K., Sultmann, H., Wilmer, F., von Heydebreck, A., Haas, S., Korn, B., Gunawan, B., Vente, A., Fuzesi, L., et al. (2001). Identification and classification of differentially expressed genes in renal cell carcinoma by expression profiling on a global human 31,500-element cDNA array. Genome research 11, 1861-1870.

Boisvert-Adamo, K., Longmate, W., Abel, E.V., and Aplin, A.E. (2009). Mcl-1 is required for melanoma cell resistance to anoikis. Mol Cancer Res 7, 549-556.

Boudreau, N., Sympson, C.J., Werb, Z., and Bissell, M.J. (1995). Suppression of ICE and apoptosis in mammary epithelial cells by extracellular matrix. Science (New York, NY 267, 891-893.

Bourguignon, L.Y. (2001). CD44-mediated oncogenic signaling and cytoskeleton activation during mammary tumor progression. Journal of mammary gland biology and neoplasia 6, 287-297. 
Bourguignon, L.Y., Gunja-Smith, Z., lida, N., Zhu, H.B., Young, L.J., Muller, W.J., and Cardiff, R.D. (1998). CD44v(3,8-10) is involved in cytoskeleton-mediated tumor cell migration and matrix metalloproteinase (MMP-9) association in metastatic breast cancer cells. Journal of cellular physiology 176, 206-215.

Bourguignon, L.Y., Lokeshwar, V.B., He, J., Chen, X., and Bourguignon, G.J. (1992). A CD44-like endothelial cell transmembrane glycoprotein (GP116) interacts with extracellular matrix and ankyrin. Molecular and cellular biology 12, 4464-4471.

Bourguignon, L.Y., Peyrollier, K., Xia, W., and Gilad, E. (2008). Hyaluronan-CD44 interaction activates stem cell marker Nanog, Stat-3-mediated MDR1 gene expression, and ankyrin-regulated multidrug efflux in breast and ovarian tumor cells. The Journal of biological chemistry 283, 17635-17651.

Bourguignon, L.Y., Singleton, P.A., Zhu, H., and Zhou, B. (2002). Hyaluronan promotes signaling interaction between CD44 and the transforming growth factor beta receptor I in metastatic breast tumor cells. The Journal of biological chemistry 277, 39703-39712.

Bourguignon, L.Y., Zhu, H., Shao, L., and Chen, Y.W. (2000). Ankyrin-Tiam1 interaction promotes Rac1 signaling and metastatic breast tumor cell invasion and migration. The Journal of cell biology 150, 177-191.

Bourguignon, L.Y., Zhu, H., Shao, L., Zhu, D., and Chen, Y.W. (1999). Rho-kinase (ROK) promotes CD44v(3,8-10)-ankyrin interaction and tumor cell migration in metastatic breast cancer cells. Cell motility and the cytoskeleton 43, 269-287.

Burridge, K., and Wennerberg, K. (2004). Rho and Rac take center stage. Cell $116,167-179$.

Camacho-Leal, P., and Stanners, C.P. (2008). The human carcinoembryonic antigen (CEA) GPI anchor mediates anoikis inhibition by inactivation of the intrinsic death pathway. Oncogene 27, 1545-1553.

Camp, R.L., Chung, G.G., and Rimm, D.L. (2002). Automated subcellular localization and quantification of protein expression in tissue microarrays. Nature medicine 8, 1323-1327.

Chinnadurai, G. (2009). The transcriptional corepressor CtBP: a foe of multiple tumor suppressors. Cancer research 69, 731-734.

Chomez, P., De Backer, O., Bertrand, M., De Plaen, E., Boon, T., and Lucas, S. (2001). An overview of the MAGE gene family with the identification of all human members of the family. Cancer research $61,5544-5551$. 
Chu, C.S., Xue, B., Tu, C., Feng, Z.H., Shi, Y.H., Miao, Y., and Wen, C.J. (2007). NRAGE suppresses metastasis of melanoma and pancreatic cancer in vitro and in vivo. Cancer letters 250, 268-275.

Collins, N.L., Reginato, M.J., Paulus, J.K., Sgroi, D.C., Labaer, J., and Brugge, J.S. (2005). G1/S cell cycle arrest provides anoikis resistance through Erk-mediated Bim suppression. Molecular and cellular biology 25, 5282-5291.

Coniglio, S.J., Jou, T.S., and Symons, M. (2001). Rac1 protects epithelial cells against anoikis. The Journal of biological chemistry 276, 28113-28120.

Davey, F., and Davies, A.M. (1998). TrkB signalling inhibits p75-mediated apoptosis induced by nerve growth factor in embryonic proprioceptive neurons. Curr Biol 8, 915-918.

Davis, E., Teng, H., Bilican, B., Parker, M.I., Liu, B., Carriera, S., Goding, C.R., and Prince, S. (2008). Ectopic Tbx2 expression results in polyploidy and cisplatin resistance. Oncogene 27, 976-984.

Del Rio, M., Imam, A., DeLeon, M., Gomez, G., Mishra, J., Ma, Q., Parikh, S., and Devarajan, P. (2004). The death domain of kidney ankyrin interacts with Fas and promotes Fas-mediated cell death in renal epithelia. J Am Soc Nephrol 15, 41-51.

Derksen, P.W., Liu, X., Saridin, F., van der Gulden, H., Zevenhoven, J., Evers, B., van Beijnum, J.R., Griffioen, A.W., Vink, J., Krimpenfort, P., et al. (2006). Somatic inactivation of E-cadherin and p53 in mice leads to metastatic lobular mammary carcinoma through induction of anoikis resistance and angiogenesis. Cancer cell 10, 437449 .

Douma, S., Van Laar, T., Zevenhoven, J., Meuwissen, R., Van Garderen, E., and Peeper, D.S. (2004). Suppression of anoikis and induction of metastasis by the neurotrophic receptor TrkB. Nature 430, 1034-1039.

Du, Q., Zhang, Y., Tian, X.X., Li, Y., and Fang, W.G. (2009). MAGE-D1 inhibits proliferation, migration and invasion of human breast cancer cells. Oncology reports 22, 659-665.

Emoto, Y. (2008). Cellular aggregation facilitates anoikis in MDCK cells. J Physiol Sci 58, 371-380.

Enari, M., Hug, H., and Nagata, S. (1995). Involvement of an ICE-like protease in Fas-mediated apoptosis. Nature $375,78-81$.

Feinstein, E., Kimchi, A., Wallach, D., Boldin, M., and Varfolomeev, E. (1995). The death domain: a module shared by proteins with diverse cellular functions. Trends in biochemical sciences $20,342-344$. 
Fouquet, S., Lugo-Martinez, V.H., Chambaz, J., Cardot, P., Pincon-Raymond, M., and Thenet, S. (2004). [Control of the survival/apoptosis balance by E-cadherin: role in enterocyte anoikis]. Journal de la Societe de biologie 198, 379-383.

Fraser, A., and Evan, G. (1996). A license to kill. Cell 85, 781-784.

Frisch, S.M. (1999). Evidence for a function of death-receptor-related, deathdomain-containing proteins in anoikis. Curr Biol 9, 1047-1049.

Frisch, S.M., and Francis, H. (1994). Disruption of epithelial cell-matrix interactions induces apoptosis. The Journal of cell biology 124, 619-626.

Frisch, S.M., and Ruoslahti, E. (1997). Integrins and anoikis. Current opinion in cell biology $9,701-706$.

Frisch, S.M., and Screaton, R.A. (2001). Anoikis mechanisms. Current opinion in cell biology $13,555-562$.

Frisch, S.M., Vuori, K., Ruoslahti, E., and Chan-Hui, P.Y. (1996). Control of adhesion-dependent cell survival by focal adhesion kinase. The Journal of cell biology 134, 793-799.

Gao, C., Sun, W., Christofidou-Solomidou, M., Sawada, M., Newman, D.K., Bergom, C., Albelda, S.M., Matsuyama, S., and Newman, P.J. (2003). PECAM-1 functions as a specific and potent inhibitor of mitochondrial-dependent apoptosis. Blood 102, 169-179.

Geiger, T.R., and Peeper, D.S. (2007). Critical role for TrkB kinase function in anoikis suppression, tumorigenesis, and metastasis. Cancer research 67, 6221-6229.

Ghosh, S., and Cox, J.V. (2001). Dynamics of ankyrin-containing complexes in chicken embryonic erythroid cells: role of phosphorylation. Molecular biology of the cell 12, 3864-3874.

Gilmore, A.P., Metcalfe, A.D., Romer, L.H., and Streuli, C.H. (2000). Integrinmediated survival signals regulate the apoptotic function of Bax through its conformation and subcellular localization. The Journal of cell biology 149, 431-446.

Gilmore, A.P., Owens, T.W., Foster, F.M., and Lindsay, J. (2009). How adhesion signals reach a mitochondrial conclusion--ECM regulation of apoptosis. Current opinion in cell biology 21, 654-661.

Ginos, M.A., Page, G.P., Michalowicz, B.S., Patel, K.J., Volker, S.E., Pambuccian, S.E., Ondrey, F.G., Adams, G.L., and Gaffney, P.M. (2004). Identification of a gene 
expression signature associated with recurrent disease in squamous cell carcinoma of the head and neck. Cancer research 64, 55-63.

Glinsky, G.V., Berezovska, O., and Glinskii, A.B. (2005). Microarray analysis identifies a death-from-cancer signature predicting therapy failure in patients with multiple types of cancer. The Journal of clinical investigation 115, 1503-1521.

Gnesutta, N., Qu, J., and Minden, A. (2001). The serine/threonine kinase PAK4 prevents caspase activation and protects cells from apoptosis. The Journal of biological chemistry $276,14414-14419$.

Gotzmann, J., Fischer, A.N., Zojer, M., Mikula, M., Proell, V., Huber, H., Jechlinger, M., Waerner, T., Weith, A., Beug, H., et al. (2006). A crucial function of PDGF in TGF-beta-mediated cancer progression of hepatocytes. Oncogene 25, 3170-3185.

Graff, J.R., Deddens, J.A., Konicek, B.W., Colligan, B.M., Hurst, B.M., Carter, H.W., and Carter, J.H. (2001). Integrin-linked kinase expression increases with prostate tumor grade. Clin Cancer Res 7, 1987-1991.

Grigo, K., Wirsing, A., Lucas, B., Klein-Hitpass, L., and Ryffel, G.U. (2008). HNF4 alpha orchestrates a set of 14 genes to down-regulate cell proliferation in kidney cells. Biological chemistry 389, 179-187.

Groeper, C., Gambazzi, F., Zajac, P., Bubendorf, L., Adamina, M., Rosenthal, R., Zerkowski, H.R., Heberer, M., and Spagnoli, G.C. (2007). Cancer/testis antigen expression and specific cytotoxic $\mathrm{T}$ lymphocyte responses in non small cell lung cancer. International journal of cancer 120, 337-343.

Grooteclaes, M.L., and Frisch, S.M. (2000). Evidence for a function of CtBP in epithelial gene regulation and anoikis. Oncogene 19, 3823-3828.

Grossmann, J. (2002). Molecular mechanisms of "detachment-induced apoptosis-Anoikis". Apoptosis 7, 247-260. 70.

Hanahan, D., and Weinberg, R.A. (2000). The hallmarks of cancer. Cell 100, 57-

Harada, O., Suga, T., Suzuki, T., Nakamoto, K., Kobayashi, M., Nomiyama, T., Nadano, D., Ohyama, C., Fukuda, M.N., and Nakayama, J. (2007). The role of trophinin, an adhesion molecule unique to human trophoblasts, in progression of colorectal cancer. International journal of cancer 121, 1072-1078.

Howlett, A.R., Bailey, N., Damsky, C., Petersen, O.W., and Bissell, M.J. (1995). Cellular growth and survival are mediated by beta 1 integrins in normal human breast epithelium but not in breast carcinoma. Journal of cell science 108 ( Pt 5), 1945-1957. 
Ignatiuk, A., Quickfall, J.P., Hawrysh, A.D., Chamberlain, M.D., and Anderson, D.H. (2006). The smaller isoforms of ankyrin 3 bind to the p85 subunit of phosphatidylinositol 3'-kinase and enhance platelet-derived growth factor receptor downregulation. The Journal of biological chemistry 281, 5956-5964.

Ilantzis, C., DeMarte, L., Screaton, R.A., and Stanners, C.P. (2002). Deregulated expression of the human tumor marker CEA and CEA family member CEACAM6 disrupts tissue architecture and blocks colonocyte differentiation. Neoplasia (New York, NY 4, 151-163.

Inoue, A., Seidel, M.G., Wu, W., Kamizono, S., Ferrando, A.A., Bronson, R.T., Iwasaki, H., Akashi, K., Morimoto, A., Hitzler, J.K., et al. (2002). Slug, a highly conserved zinc finger transcriptional repressor, protects hematopoietic progenitor cells from radiation-induced apoptosis in vivo. Cancer cell 2, 279-288.

Ipsaro, J.J., Huang, L., Gutierrez, L., and MacDonald, R.I. (2008). Molecular epitopes of the ankyrin-spectrin interaction. Biochemistry 47, 7452-7464.

Ipsaro, J.J., Huang, L., and Mondragon, A. (2009). Structures of the spectrinankyrin interaction binding domains. Blood 113, 5385-5393.

Ismail, A., and Bateman, A. (2009). Expression of TBX2 promotes anchorageindependent growth and survival in the p53-negative SW13 adrenocortical carcinoma. Cancer letters 278, 230-240.

Jaboin, J., Kim, C.J., Kaplan, D.R., and Thiele, C.J. (2002). Brain-derived neurotrophic factor activation of TrkB protects neuroblastoma cells from chemotherapyinduced apoptosis via phosphatidylinositol 3'-kinase pathway. Cancer research 62, 67566763.

Jacobs, J.J., Keblusek, P., Robanus-Maandag, E., Kristel, P., Lingbeek, M., Nederlof, P.M., van Welsem, T., van de Vijver, M.J., Koh, E.Y., Daley, G.Q., et al. (2000). Senescence bypass screen identifies TBX2, which represses Cdkn2a (p19(ARF)) and is amplified in a subset of human breast cancers. Nature genetics 26, 291-299.

Juliano, R.L., and Varner, J.A. (1993). Adhesion molecules in cancer: the role of integrins. Current opinion in cell biology 5, 812-818.

Kalluri, R., and Weinberg, R.A. (2009). The basics of epithelial-mesenchymal transition. The Journal of clinical investigation 119, 1420-1428.

Kalomiris, E.L., and Bourguignon, L.Y. (1989). Lymphoma protein kinase C is associated with the transmembrane glycoprotein, GP85, and may function in GP85ankyrin binding. The Journal of biological chemistry $264,8113-8119$. 
Kamarajan, P., Bunek, J., Lin, Y., Nunez, G., and Kapila, Y.L. Receptor-interacting protein shuttles between cell death and survival signaling pathways. Molecular biology of the cell $21,481-488$.

Kamb, A., Gruis, N.A., Weaver-Feldhaus, J., Liu, Q., Harshman, K., Tavtigian, S.V., Stockert, E., Day, R.S., 3rd, Johnson, B.E., and Skolnick, M.H. (1994). A cell cycle regulator potentially involved in genesis of many tumor types. Science (New York, NY 264, 436-440.

Keely, P.J., Westwick, J.K., Whitehead, I.P., Der, C.J., and Parise, L.V. (1997). Cdc42 and Rac1 induce integrin-mediated cell motility and invasiveness through $\mathrm{PI}(3) \mathrm{K}$. Nature 390, 632-636.

Kizhatil, K., Baker, S.A., Arshavsky, V.Y., and Bennett, V. (2009). Ankyrin-G promotes cyclic nucleotide-gated channel transport to rod photoreceptor sensory cilia. Science (New York, NY 323, 1614-1617.

Kizhatil, K., and Bennett, V. (2004). Lateral membrane biogenesis in human bronchial epithelial cells requires 190-kDa ankyrin-G. The Journal of biological chemistry 279, 16706-16714.

Kizhatil, K., Davis, J.Q., Davis, L., Hoffman, J., Hogan, B.L., and Bennett, V. (2007). Ankyrin-G is a molecular partner of E-cadherin in epithelial cells and early embryos. The Journal of biological chemistry 282, 26552-26561.

Knudson, W. (1996). Tumor-associated hyaluronan. Providing an extracellular matrix that facilitates invasion. The American journal of pathology 148, 1721-1726.

Kolondra, A., Grzybek, M., Chorzalska, A., and Sikorski, A.F. (2008). The $22.5 \mathrm{kDa}$ spectrin-binding domain of ankyrinR binds spectrin with high affinity and changes the spectrin distribution in cells in vivo. Protein expression and purification 60, 157-164.

Kordeli, E., Lambert, S., and Bennett, V. (1995). AnkyrinG. A new ankyrin gene with neural-specific isoforms localized at the axonal initial segment and node of Ranvier. The Journal of biological chemistry 270, 2352-2359.

Kovacevic, Z., and Richardson, D.R. (2006). The metastasis suppressor, Ndrg-1: a new ally in the fight against cancer. Carcinogenesis 27, 2355-2366.

Kurenova, E., Xu, L.H., Yang, X., Baldwin, A.S., Jr., Craven, R.J., Hanks, S.K., Liu, Z.G., and Cance, W.G. (2004). Focal adhesion kinase suppresses apoptosis by binding to the death domain of receptor-interacting protein. Molecular and cellular biology 24 , 4361-4371. 
Kuwajima, T., Taniura, H., Nishimura, I., and Yoshikawa, K. (2004). Necdin interacts with the Msx2 homeodomain protein via MAGE-D1 to promote myogenic differentiation of C2C12 cells. The Journal of biological chemistry 279, 40484-40493.

Lassus, P., Opitz-Araya, X., and Lazebnik, Y. (2002). Requirement for caspase-2 in stress-induced apoptosis before mitochondrial permeabilization. Science (New York, NY 297, 1352-1354.

Law, S.F., O'Neill, G.M., Fashena, S.J., Einarson, M.B., and Golemis, E.A. (2000). The docking protein HEF1 is an apoptotic mediator at focal adhesion sites. Molecular and cellular biology 20, 5184-5195.

Lee, G., Abdi, K., Jiang, Y., Michaely, P., Bennett, V., and Marszalek, P.E. (2006). Nanospring behaviour of ankyrin repeats. Nature 440, 246-249.

Lei, K., and Davis, R.J. (2003). JNK phosphorylation of Bim-related members of the Bcl2 family induces Bax-dependent apoptosis. Proceedings of the National Academy of Sciences of the United States of America 100, 2432-2437.

Ley, R., Ewings, K.E., Hadfield, K., Howes, E., Balmanno, K., and Cook, S.J. (2004). Extracellular signal-regulated kinases 1/2 are serum-stimulated "Bim(EL) kinases" that bind to the $\mathrm{BH} 3$-only protein $\mathrm{Bim}(\mathrm{EL})$ causing its phosphorylation and turnover. The Journal of biological chemistry $279,8837-8847$.

Li, Q.Q., Xu, J.D., Wang, W.J., Cao, X.X., Chen, Q., Tang, F., Chen, Z.Q., Liu, X.P., and Xu, Z.D. (2009). Twist1-mediated adriamycin-induced epithelial-mesenchymal transition relates to multidrug resistance and invasive potential in breast cancer cells. Clin Cancer Res 15, 2657-2665.

Lin, Y., Devin, A., Rodriguez, Y., and Liu, Z.G. (1999). Cleavage of the death domain kinase RIP by caspase-8 prompts TNF-induced apoptosis. Genes \& development 13, 2514-2526.

Lokeshwar, V.B., Fregien, N., and Bourguignon, L.Y. (1994). Ankyrin-binding domain of CD44(GP85) is required for the expression of hyaluronic acid-mediated adhesion function. The Journal of cell biology 126, 1099-1109.

Lugo-Martinez, V.H., Petit, C.S., Fouquet, S., Le Beyec, J., Chambaz, J., PinconRaymond, M., Cardot, P., and Thenet, S. (2009). Epidermal growth factor receptor is involved in enterocyte anoikis through the dismantling of E-cadherin-mediated junctions. American journal of physiology 296, G235-244.

Mani, S.A., Guo, W., Liao, M.J., Eaton, E.N., Ayyanan, A., Zhou, A.Y., Brooks, M., Reinhard, F., Zhang, C.C., Shipitsin, M., et al. (2008). The epithelial-mesenchymal transition generates cells with properties of stem cells. Cell 133, 704-715. 
Masuda, Y., Sasaki, A., Shibuya, H., Ueno, N., Ikeda, K., and Watanabe, K. (2001). Dlxin-1, a novel protein that binds DIx5 and regulates its transcriptional function. The Journal of biological chemistry 276, 5331-5338.

Matluk, N., Rochira, J.A., Karaczyn, A., Adams, T., and Verdi, J.M. A role for NRAGE in NF-kappaB activation through the non-canonical BMP pathway. BMC biology $8,7$.

Matsuda, T., Suzuki, H., Oishi, I., Kani, S., Kuroda, Y., Komori, T., Sasaki, A., Watanabe, K., and Minami, Y. (2003). The receptor tyrosine kinase Ror2 associates with the melanoma-associated antigen (MAGE) family protein Dlxin-1 and regulates its intracellular distribution. The Journal of biological chemistry 278, 29057-29064.

Meredith, J.E., Jr., Fazeli, B., and Schwartz, M.A. (1993). The extracellular matrix as a cell survival factor. Molecular biology of the cell 4, 953-961.

Michaely, P., Tomchick, D.R., Machius, M., and Anderson, R.G. (2002). Crystal structure of a 12 ANK repeat stack from human ankyrinR. The EMBO journal 21, 63876396.

Miller, C.T., Chen, G., Gharib, T.G., Wang, H., Thomas, D.G., Misek, D.E., Giordano, T.J., Yee, J., Orringer, M.B., Hanash, S.M., et al. (2003). Increased C-CRK proto-oncogene expression is associated with an aggressive phenotype in lung adenocarcinomas. Oncogene 22, 7950-7957.

Mills, K.R., Reginato, M., Debnath, J., Queenan, B., and Brugge, J.S. (2004). Tumor necrosis factor-related apoptosis-inducing ligand (TRAIL) is required for induction of autophagy during lumen formation in vitro. Proceedings of the National Academy of Sciences of the United States of America 101, 3438-3443.

Mohler, P.J., and Bennett, V. (2005). Defects in ankyrin-based cellular pathways in metazoan physiology. Front Biosci 10, 2832-2840.

Mohler, P.J., Gramolini, A.O., and Bennett, V. (2002). Ankyrins. Journal of cell science 115, 1565-1566.

Mohler, P.J., Yoon, W., and Bennett, V. (2004). Ankyrin-B targets beta2-spectrin to an intracellular compartment in neonatal cardiomyocytes. The Journal of biological chemistry $279,40185-40193$.

Nelson, W.J., Shore, E.M., Wang, A.Z., and Hammerton, R.W. (1990). Identification of a membrane-cytoskeletal complex containing the cell adhesion molecule uvomorulin (E-cadherin), ankyrin, and fodrin in Madin-Darby canine kidney epithelial cells. The Journal of cell biology 110, 349-357. 
Newman, P.J. (1997). The biology of PECAM-1. The Journal of clinical investigation 99, 3-8.

Onder, T.T., Gupta, P.B., Mani, S.A., Yang, J., Lander, E.S., and Weinberg, R.A. (2008). Loss of E-cadherin promotes metastasis via multiple downstream transcriptional pathways. Cancer research 68, 3645-3654.

Passananti, C., and Fanciulli, M. (2007). The anti-apoptotic factor Che-1/AATF links transcriptional regulation, cell cycle control, and DNA damage response. Cell division 2, 21.

Paxton, C., Zhao, H., Chin, Y., Langner, K., and Reecy, J. (2002). Murine Tbx2 contains domains that activate and repress gene transcription. Gene 283, 117-124.

Perez-Losada, J., Sanchez-Martin, M., Perez-Caro, M., Perez-Mancera, P.A., and Sanchez-Garcia, I. (2003). The radioresistance biological function of the SCF/kit signaling pathway is mediated by the zinc-finger transcription factor Slug. Oncogene 22, 42054211.

Perez-Moreno, M., Jamora, C., and Fuchs, E. (2003). Sticky business: orchestrating cellular signals at adherens junctions. Cell 112, 535-548.

Playford, M.P., Vadali, K., Cai, X., Burridge, K., and Schaller, M.D. (2008). Focal adhesion kinase regulates cell-cell contact formation in epithelial cells via modulation of Rho. Experimental cell research 314, 3187-3197.

Pold, M., Zhou, J., Chen, G.L., Hall, J.M., Vescio, R.A., and Berenson, J.R. (1999). Identification of a new, unorthodox member of the MAGE gene family. Genomics 59, 161167.

Ponta, H., Sherman, L., and Herrlich, P.A. (2003). CD44: from adhesion molecules to signalling regulators. Nature reviews 4, 33-45.

Prince, S., Carreira, S., Vance, K.W., Abrahams, A., and Goding, C.R. (2004). Tbx2 directly represses the expression of the p21(WAF1) cyclin-dependent kinase inhibitor. Cancer research 64, 1669-1674.

Puthalakath, H., Villunger, A., O'Reilly, L.A., Beaumont, J.G., Coultas, L., Cheney, R.E., Huang, D.C., and Strasser, A. (2001). Bmf: a proapoptotic BH3-only protein regulated by interaction with the myosin $\mathrm{V}$ actin motor complex, activated by anoikis. Science (New York, NY 293, 1829-1832.

Qu, J., Cammarano, M.S., Shi, Q., Ha, K.C., de Lanerolle, P., and Minden, A. (2001). Activated PAK4 regulates cell adhesion and anchorage-independent growth. Molecular and cellular biology 21, 3523-3533. 
Rastaldi, M.P., Ferrario, F., Giardino, L., Dell'Antonio, G., Grillo, C., Grillo, P., Strutz, F., Muller, G.A., Colasanti, G., and D'Amico, G. (2002). Epithelial-mesenchymal transition of tubular epithelial cells in human renal biopsies. Kidney international 62, 137146.

Redmond, K.L., Crawford, N.T., Farmer, H., D'Costa, Z.C., O'Brien, G.J., Buckley, N.E., Kennedy, R.D., Johnston, P.G., Harkin, D.P., and Mullan, P.B. T-box 2 represses NDRG1 through an EGR1-dependent mechanism to drive the proliferation of breast cancer cells. Oncogene.

Reginato, M.J., Mills, K.R., Paulus, J.K., Lynch, D.K., Sgroi, D.C., Debnath, J., Muthuswamy, S.K., and Brugge, J.S. (2003). Integrins and EGFR coordinately regulate the pro-apoptotic protein Bim to prevent anoikis. Nature cell biology 5, 733-740.

Rodriguez Fernandez, J.L., Geiger, B., Salomon, D., and Ben-Ze'ev, A. (1993). Suppression of vinculin expression by antisense transfection confers changes in cell morphology, motility, and anchorage-dependent growth of 3T3 cells. The Journal of cell biology 122, 1285-1294.

Rodriguez, M., Aladowicz, E., Lanfrancone, L., and Goding, C.R. (2008). Tbx3 represses E-cadherin expression and enhances melanoma invasiveness. Cancer research $68,7872-7881$.

Rubtsov, A.M., and Lopina, O.D. (2000). Ankyrins. FEBS letters 482, 1-5.

Rush, J., Moritz, A., Lee, K.A., Guo, A., Goss, V.L., Spek, E.J., Zhang, H., Zha, X.M., Polakiewicz, R.D., and Comb, M.J. (2005). Immunoaffinity profiling of tyrosine phosphorylation in cancer cells. Nature biotechnology 23, 94-101.

Rytomaa, M., Martins, L.M., and Downward, J. (1999). Involvement of FADD and caspase-8 signalling in detachment-induced apoptosis. Curr Biol 9, 1043-1046.

Saito, S., Yamaguchi, H., Higashimoto, Y., Chao, C., Xu, Y., Fornace, A.J., Jr., Appella, E., and Anderson, C.W. (2003). Phosphorylation site interdependence of human p53 post-translational modifications in response to stress. The Journal of biological chemistry $278,37536-37544$.

Salehi, A.H., Roux, P.P., Kubu, C.J., Zeindler, C., Bhakar, A., Tannis, L.L., Verdi, J.M., and Barker, P.A. (2000). NRAGE, a novel MAGE protein, interacts with the p75 neurotrophin receptor and facilitates nerve growth factor-dependent apoptosis. Neuron $27,279-288$.

Salehi, A.H., Xanthoudakis, S., and Barker, P.A. (2002). NRAGE, a p75 neurotrophin receptor-interacting protein, induces caspase activation and cell death through a JNK-dependent mitochondrial pathway. The Journal of biological chemistry $277,48043-48050$. 
Samara, R.N., Laguinge, L.M., and Jessup, J.M. (2007). Carcinoembryonic antigen inhibits anoikis in colorectal carcinoma cells by interfering with TRAIL-R2 (DR5) signaling. Cancer research 67, 4774-4782.

Sasaki, A., Hinck, L., and Watanabe, K. (2005). RumMAGE-D the members: structure and function of a new adaptor family of MAGE-D proteins. Journal of receptor and signal transduction research 25, 181-198.

Sasaki, A., Masuda, Y., Iwai, K., Ikeda, K., and Watanabe, K. (2002). A RING finger protein Praja1 regulates Dlx5-dependent transcription through its ubiquitin ligase activity for the Dlx/Msx-interacting MAGE/Necdin family protein, Dlxin-1. The Journal of biological chemistry 277, 22541-22546.

Schafer, Z.T., Grassian, A.R., Song, L., Jiang, Z., Gerhart-Hines, Z., Irie, H.Y., Gao, S., Puigserver, P., and Brugge, J.S. (2009). Antioxidant and oncogene rescue of metabolic defects caused by loss of matrix attachment. Nature 461, 109-113.

Schmelzle, T., Mailleux, A.A., Overholtzer, M., Carroll, J.S., Solimini, N.L., Lightcap, E.S., Veiby, O.P., and Brugge, J.S. (2007). Functional role and oncogeneregulated expression of the $\mathrm{BH} 3-$ only factor Bmf in mammary epithelial anoikis and morphogenesis. Proceedings of the National Academy of Sciences of the United States of America 104, 3787-3792.

Schurmann, A., Mooney, A.F., Sanders, L.C., Sells, M.A., Wang, H.G., Reed, J.C., and Bokoch, G.M. (2000). p21-activated kinase 1 phosphorylates the death agonist bad and protects cells from apoptosis. Molecular and cellular biology 20,453-461.

Shirai, M., Imanaka-Yoshida, K., Schneider, M.D., Schwartz, R.J., and Morisaki, T. (2009). T-box 2, a mediator of Bmp-Smad signaling, induced hyaluronan synthase 2 and Tgfbeta2 expression and endocardial cushion formation. Proceedings of the National Academy of Sciences of the United States of America 106, 18604-18609.

Simpson, C.D., Mawji, I.A., Anyiwe, K., Williams, M.A., Wang, X., Venugopal, A.L., Gronda, M., Hurren, R., Cheng, S., Serra, S., et al. (2009). Inhibition of the sodium potassium adenosine triphosphatase pump sensitizes cancer cells to anoikis and prevents distant tumor formation. Cancer research 69, 2739-2747.

Smit, M.A., Geiger, T.R., Song, J.Y., Gitelman, I., and Peeper, D.S. (2009). A Twist-Snail axis critical for TrkB-induced epithelial-mesenchymal transition-like transformation, anoikis resistance, and metastasis. Molecular and cellular biology 29, 3722-3737.

Smith, J.J., Richardson, D.A., Kopf, J., Yoshida, M., Hollingsworth, R.E., and Kornbluth, S. (2002). Apoptotic regulation by the Crk adapter protein mediated by interactions with Wee1 and Crm1/exportin. Molecular and cellular biology 22, 1412-1423. 
Stockinger, A., Eger, A., Wolf, J., Beug, H., and Foisner, R. (2001). E-cadherin regulates cell growth by modulating proliferation-dependent beta-catenin transcriptional activity. The Journal of cell biology 154, 1185-1196.

Subauste, M.C., Pertz, O., Adamson, E.D., Turner, C.E., Junger, S., and Hahn, K.M. (2004). Vinculin modulation of paxillin-FAK interactions regulates ERK to control survival and motility. The Journal of cell biology 165, 371-381.

Thiele, C.J., Li, Z., and McKee, A.E. (2009). On Trk--the TrkB signal transduction pathway is an increasingly important target in cancer biology. Clin Cancer Res 15, 59625967.

Thiery, J.P. (2002). Epithelial-mesenchymal transitions in tumour progression. Nature reviews 2, 442-454.

Toole, B.P. (2004). Hyaluronan: from extracellular glue to pericellular cue. Nat Rev Cancer 4, 528-539.

Vega, S., Morales, A.V., Ocana, O.H., Valdes, F., Fabregat, I., and Nieto, M.A. (2004). Snail blocks the cell cycle and confers resistance to cell death. Genes \& development 18, 1131-1143.

Vicovac, L., and Aplin, J.D. (1996). Epithelial-mesenchymal transition during trophoblast differentiation. Acta anatomica 156, 202-216.

Vormer, T.L., Foijer, F., Wielders, C.L., and te Riele, H. (2008). Anchorageindependent growth of pocket protein-deficient murine fibroblasts requires bypass of $\mathrm{G} 2$ arrest and can be accomplished by expression of TBX2. Molecular and cellular biology $28,7263-7273$.

Wang, J., Robinson, J.F., O'Neil, C.H., Edwards, J.Y., Williams, C.M., Huff, M.W., Pickering, J.G., and Hegele, R.A. (2006). Ankyrin G overexpression in Hutchinson-Gilford progeria syndrome fibroblasts identified through biological filtering of expression profiles. Journal of human genetics 51, 934-942.

Wang, R., Wei, Z., Jin, H., Wu, H., Yu, C., Wen, W., Chan, L.N., Wen, Z., and Zhang, M. (2009). Autoinhibition of UNC5b revealed by the cytoplasmic domain structure of the receptor. Molecular cell 33, 692-703.

Wang, X., Ling, M.T., Guan, X.Y., Tsao, S.W., Cheung, H.W., Lee, D.T., and Wong, Y.C. (2004). Identification of a novel function of TWIST, a bHLH protein, in the development of acquired taxol resistance in human cancer cells. Oncogene 23, 474-482.

Wei, L., Yang, Y., Zhang, X., and Yu, Q. (2002). Anchorage-independent phosphorylation of $\mathrm{p} 130$ (Cas) protects lung adenocarcinoma cells from anoikis. Journal of cellular biochemistry $87,439-449$. 
Wei, L., Yang, Y., Zhang, X., and Yu, Q. (2004). Cleavage of p130Cas in anoikis. Journal of cellular biochemistry 91, 325-335.

Weihua, Z., Tsan, R., Huang, W.C., Wu, Q., Chiu, C.H., Fidler, I.J., and Hung, M.C. (2008). Survival of cancer cells is maintained by EGFR independent of its kinase activity. Cancer cell 13, 385-393.

Weinberg, R.A. (2008). Twisted epithelial-mesenchymal transition blocks senescence. Nature cell biology 10, 1021-1023.

Wellner, U., Schubert, J., Burk, U.C., Schmalhofer, O., Zhu, F., Sonntag, A., Waldvogel, B., Vannier, C., Darling, D., zur Hausen, A., et al. (2009). The EMT-activator ZEB1 promotes tumorigenicity by repressing stemness-inhibiting microRNAs. Nature cell biology $11,1487-1495$.

Welsh, C.F., Zhu, D., and Bourguignon, L.Y. (1995). Interaction of CD44 variant isoforms with hyaluronic acid and the cytoskeleton in human prostate cancer cells. Journal of cellular physiology 164, 605-612.

Williams, M.E., Strickland, P., Watanabe, K., and Hinck, L. (2003). UNC5H1 induces apoptosis via its juxtamembrane region through an interaction with NRAGE. The Journal of biological chemistry $278,17483-17490$.

Woroniecki, R., Ferdinand, J.R., Morrow, J.S., and Devarajan, P. (2003). Dissociation of spectrin-ankyrin complex as a basis for loss of Na-K-ATPase polarity after ischemia. Am J Physiol Renal Physiol 284, F358-364.

Wu, Y., Dowbenko, D., Pisabarro, M.T., Dillard-Telm, L., Koeppen, H., and Lasky, L.A. (2001). PTEN 2, a Golgi-associated testis-specific homologue of the PTEN tumor suppressor lipid phosphatase. The Journal of biological chemistry 276, 21745-21753.

Xue, B., Wen, C., Shi, Y., Zhao, D., and Li, C. (2005). Human NRAGE disrupts Ecadherin/beta-catenin regulated homotypic cell-cell adhesion. Biochemical and biophysical research communications 336, 247-251.

Yang, B., Wu, J., Maddodi, N., Ma, Y., Setaluri, V., and Longley, B.J. (2007). Epigenetic control of MAGE gene expression by the KIT tyrosine kinase. The Journal of investigative dermatology 127, 2123-2128.

Yu, Q., Toole, B.P., and Stamenkovic, I. (1997). Induction of apoptosis of metastatic mammary carcinoma cells in vivo by disruption of tumor cell surface CD44 function. The Journal of experimental medicine 186, 1985-1996.

Zahir, N., Lakins, J.N., Russell, A., Ming, W., Chatterjee, C., Rozenberg, G.I., Marinkovich, M.P., and Weaver, V.M. (2003). Autocrine laminin-5 ligates alpha6beta4 
integrin and activates RAC and NFkappaB to mediate anchorage-independent survival of mammary tumors. The Journal of cell biology 163, 1397-1407.

Zhang, C.G., Xing, G.C., Wei, H.D., Yu, Y.T., and He, F.C. (2001). [A new melanoma antigen-encoding gene subfamily in human chromosome $\mathrm{X}$ ]. Yi chuan xue bao $=$ Acta genetica Sinica 28, 197-203.

Zhang, X., and Bennett, V. (1998). Restriction of 480/270-kD ankyrin G to axon proximal segments requires multiple ankyrin G-specific domains. The Journal of cell biology 142, 1571-1581.

Zhang, X., Davis, J.Q., Carpenter, S., and Bennett, V. (1998). Structural requirements for association of neurofascin with ankyrin. The Journal of biological chemistry $273,30785-30794$.

Zhang, X., Xu, L.H., and Yu, Q. Cell aggregation induces phosphorylation of PECAM-1 and Pyk2 and promotes tumor cell anchorage-independent growth. Molecular cancer 9,7 .

Zhang, Y., Lu, H., Dazin, P., and Kapila, Y. (2004). Squamous cell carcinoma cell aggregates escape suspension-induced, p53-mediated anoikis: fibronectin and integrin alphav mediate survival signals through focal adhesion kinase. The Journal of biological chemistry $279,48342-48349$.

Zhang, Z., Han, L., Cao, L., Liang, X., Liu, Y., Liu, H., Du, J., Qu, Z., Zhu, C., Liu, S., et al. (2008). Aggregation formation mediated anoikis resistance of BEL7402 hepatoma cells. Folia histochemica et cytobiologica / Polish Academy of Sciences, Polish Histochemical and Cytochemical Society 46, 331-336.

Zhou, D., Lambert, S., Malen, P.L., Carpenter, S., Boland, L.M., and Bennett, V. (1998). Ankyring is required for clustering of voltage-gated $\mathrm{Na}$ channels at axon initial segments and for normal action potential firing. The Journal of cell biology 143, 12951304.

Zhu, D., and Bourguignon, L.Y. (1998). The ankyrin-binding domain of CD44s is involved in regulating hyaluronic acid-mediated functions and prostate tumor cell transformation. Cell motility and the cytoskeleton 39, 209-222.

Zhu, D., and Bourguignon, L.Y. (2000). Interaction between CD44 and the repeat domain of ankyrin promotes hyaluronic acid-mediated ovarian tumor cell migration. Journal of cellular physiology 183, 182-195. 


\section{SANJEEV KUMAR}

\section{Curriculum Vitae}

2833 MBRCC

1 Medical Center Drive, School of Medicine, WVU

Morgantown, WV 26506

Cell \# 513-504-0312

skumar@hsc.wvu.edu

Education:

Ph.D. Biochemistry and Molecular Biology, Department of Biochemistry, School of Medicine, West Virginia University, Morgantown, WV Dissertation: "Regulation of anoikis by ankyrin complexes" Advisor: Steven M. Frisch, Ph.D.

M.Sc. Biotechnology, Maharaja Sayajirao University of Baroda, Baroda, India May 1999 Thesis: "Development of biopesticides from the gut microflora of rice pest" Advisor: Johannes M. Manjrekar, Ph.D.

Diploma in Biotechnology, Madurai Kamaraj University, Madurai, India B.Sc. (Honors), Chemistry, Magadh University, Bodh Gaya, India

\section{Work Experience:}

Research Associate, Avesthagen Technologies, Bangalore, India

- Cloning, expression and characterization of differentially expressed rice genes

- Incharge of genomics group, Incharge of ABI 377 sequencer, Bacterial Artificial Chromosome (BAC) libraries, genomic probes, inhouse and contractual samples for sequencing and genotyping

- Proposed, defended and initiated research work on "Characterization of aroma locus using the BAC library of Rice 2001-2002

Technical Assistant, Molecular Biology Unit, Institute of Medical Sciences, Banaras Hindu University, Varanasi, India

- Optimization of protein purification using wheat for crystallization purposes

- Assisting Dr. G.P.Pal for administrative duties 
Teaching Experience:

Graduate Teaching Assistant, Department of Biological Sciences, Wayne State University, Detroit, MI

Fall 2002-2003

- Duties included teaching the lab containing $\sim 40$ students (2 times in a week), demonstrating the lab work, setting and evaluating exams/assignments. (Biochemistry lab - Fall 2002, Microbiology lab - Winter, Summer and Fall 2003)

Honors:

2007 Best poster award (Abstract title: The cortical cytoskeletal protein ankyrinG regulates anoikis), Van Liere Memorial Convocation 2007, Health Science Center, West Virginia University

2000 Council for Scientific and Industrial Research (CSIR) fellowship, sponsored by Govt. of India

1999 Eligibility for lecturership by University Grants Commission (UGC), Govt. of India

1997-1999 Fellowship for pursuing M.Sc. (Biotechnology) by Department of Biotechnology, Govt. of India.

Publications:

Sanjeev Kumar ${ }^{*}$, Sun Hee Park, Jane Schupp, Ryan Ice, Elizabeth Killiam, David Rimm, and Steven M. Frisch. NRAGE interactions with TBX2 and ankyrin-G: a pathway for the control of anoikis by E-cadherin and EMT (In preparation for resubmission to Molecular Cell, May 2010)

Meetings and Conferences:

Podium Presentations

2009 Kumar, S., Schupp, J., Ice R., Park S. and Frisch, S. Regulation of anoikis by EMT through ankyrin complex. $12^{\text {th }}$ Translational Research Cancer Center Consortium Meeting: Biologic Therapies for Cancer 09, Abramson Cancer Center, Philadelphia, PA

2008 Kumar, S., Schupp, J., Ice R. and Frisch, S. The Role of ankyrin complexes in anoikis. The American Society for Cell Biology (ASCB), Annual Meeting 2008, San Francisco, California (Presented by my advisor Steven M. Frisch).

2007 Kumar, S. and Frisch, S. The cortical cytoskeletal protein ankyrin-G regulates anoikis. E.J. Van Liere Research Convocation, WVU. Van Liere Memorial Convocation, Health Sciences Center, West Virginia University.

\section{Poster Presentations}

2007 Kumar, S. and Frisch, S. NRAGE/MAGE-D1 Interacts with ankyrin-G and regulates anoikis. The American Society for Cell Biology (ASCB), Washington, D.C. 
2007 Kumar, S. and Frisch, S. The cortical cytoskeletal protein ankyrin-G regulates anoikis. West Virginia University Student Research day, Morgantown, WV.

2007 Kumar, S. and Frisch, S. The cortical cytoskeletal protein ankyrin-G regulates anoikis. Van Liere Memorial Convocation, Health Sciences Center, WVU, Morgantown.

2006 Kumar, S. and Frisch, S. Regulation of ankyrin-G by NRAGE/MAGE-D1.Van Liere Memorial Convocation, Health Sciences Center, WVU, Morgantown.

2001 Kumar, S., Nirmal R. and Patell V.M. Targeting enhanced quality and environmental traits from the BAC libraries of Indica rice (Basmati and Swarna) The Society for In vitro Biology, St Louis, USA.

Technical Skills:

- Automated sequencing, genotyping and analysis using the ABI 377 instrument.

- Handling and screening of High Density BAC clones and filters for structural genomics.

- Cloning and expression of genes in bacteria, yeast and mammalian cells.

- Protein, RNA and DNA purification from bacterial, plant and animal cells.

- Message analysis using quantitative RT-PCR.

- Protein-Protein interaction (Yeast 2-hybrid, Mammalian 2-hybrid, in vitro pulldown, co-immunoprecipitation.

- Protein analysis (Immunoblots and ELISA).

- Animal tissue culture (normal and cancer human cells, mouse, rat and canine cells).

- Protein over-expression (Transient and stable transfections using regular and retroviral constructs).

- Gene knock-down (siRNA/shRNA, transient and stable).

- Apoptosis analysis (caspase activity, DNA fragmentation etc).

- Fluorescence Microscopy and image analysis using Image J software.

- Luciferase assay (Single and dual).

- ECIS (electric cell substrate impedance sensing).

Professional Affiliations:

2007- Present: The American Society for Cell Biology (ASCB)

2009- Present: The Translational Research Cancer Center Consortium - Eastern Region

International Patent Applications;

2001: DNA Sequencing of ABC Transporter or Multi-Drug Resistance protein for better environment stress tolerant crops. 


\section{References:}

Dr. Steven M. Frisch

sfrisch@hsc.wvu.edu

304-293-2980

Professor, Mary Babb Randolph Cancer Center, School of Medicine, West Virginia University, Morgantown

Dr. Lisa M. Salati

Isalati@hsc.wvu.edu

304-293-7759

Vice Chair and Professor, Department of Biochemistry, School of Medicine, West Virginia University, Morgantown

Dr. Michael D. Schaller

mschaller@hsc.wvu.edu

304-293-9514

Chair and Professor, Department of Biochemistry, School of Medicine, West Virginia University, Morgantown 